Análise e melhoramento do método variacional para controle ótimo de sistemas lineares com saltos markovianos sem observação da variável de salto

\title{
Junior Rodrigues Ribeiro
}

Dissertação de Mestrado do Programa de Pós-Graduação em Ciências de Computação e Matemática Computacional (PPG-CCMC) 

Assinatura:

\section{Junior Rodrigues Ribeiro}

\section{Análise e melhoramento do método variacional para controle ótimo de sistemas lineares com saltos markovianos sem observação da variável de salto}

\footnotetext{
Dissertação apresentada ao Instituto de Ciências Matemáticas e de Computação - ICMC-USP, como parte dos requisitos para obtenção do título de Mestre em Ciências - Ciências de Computação e Matemática Computacional. VERSÃO REVISADA

Área de Concentração: Ciências de Computação e Matemática Computacional

Orientador: Prof. Dr. Eduardo Fontoura Costa
}

\section{USP - São Carlos}

Julho de 2019 
Ficha catalográfica elaborada pela Biblioteca Prof. Achille Bassi e Seção Técnica de Informática, ICMC/USP, com os dados inseridos pelo(a) autor(a)

Ribeiro, Junior Rodrigues Análise e melhoramento do método variacional para controle ótimo de sistemas lineares com saltos markovianos sem observação da variável de salto / Junior Rodrigues Ribeiro; orientador Eduardo Fontoura Costa. -- São Carlos, 2019. $98 \mathrm{p}$.

Dissertação (Mestrado - Programa de Pós-Graduação em Ciências de Computação e Matemática

Computacional) -- Instituto de Ciências Matemáticas e de Computação, Universidade de São Paulo, 2019.

1. Controle ótimo. 2. Sistemas dinâmicos lineares. 3. Cadeias de Markov. 4. Parâmetros com salto. 5. Condicionamento numérico. I. Costa, Eduardo

Fontoura, orient. II. Título.

Bibliotecários responsáveis pela estrutura de catalogação da publicação de acordo com a AACR2: Gláucia Maria Saia Cristianini - CRB - 8/4938

Juliana de Souza Moraes - CRB - 8/6176 


\section{Junior Rodrigues Ribeiro}

\section{Analysis and improvement of the variational method for control of Markov jump linear systems with no jump observation}

Master dissertation submitted to the Institute of Mathematics and Computer Sciences - ICMC-USP, in partial fulfillment of the requirements for the degree of the Master Program in Computer Science and Computational Mathematics. FINAL VERSION

Concentration Area: Computer Science and Computational Mathematics

Advisor: Prof. Dr. Eduardo Fontoura Costa

\section{USP - São Carlos}

July 2019 

Dedico aos meus pais, irmãos, minha avó e em especial à minha irmã caçula, Nayana, quem muito estimo e desejo sucesso! 

O primeiro agradecimento, nada mais justo, é ao Senhor Deus, que me deu saúde e me proporcionou esta oportunidade.

Menciono minha gratidão à Irmã Nena, que mesmo com sua idade anciã, concedeu apoio nas horas solitárias, com os assuntos vagos para rirmos e nos divertirmos, além dos muitos almoços e cafés que tomamos juntos e da moradia à sua vizinhança concedida.

À professora Marina Andretta, que me ajudou com algumas dicas de otimização nãolinear, as quais não foram diretamente empregadas no presente trabalho, mas foram de fundamental importância para que pudéssemos compreender mais a fundo o problema aqui tratado.

Ao meu orientador, professor Eduardo, que tem direcionado o meu caminho até o final desta pesquisa, fazendo-me crescer como pessoa e como pesquisador.

O presente trabalho foi realizado com apoio da Coordenação de Aperfeiçoamento de Pessoal de Nível Superior - Brasil (CAPES) - Código de Financiamento 001. Essa bolsa faz parte do projeto PICME da OBMEP, na qual fui medalhista de bronze em sua $7^{\text {a }}$ Edição (2011). 

"Disse o néscio no seu coração: Não há Deus.

Têm-se corrompido, fazem-se abomináveis em suas obras, não há ninguém que faça o bem." (Bíblia Sagrada. Salmos 14:1.) 



\section{RESUMO}

RIBEIRO, J. R. Análise e melhoramento do método variacional para controle ótimo de sistemas lineares com saltos markovianos sem observação da variável de salto. 2019. 98 p. Dissertação (Mestrado em Ciências - Ciências de Computação e Matemática Computacional) Instituto de Ciências Matemáticas e de Computação, Universidade de São Paulo, São Carlos SP, 2019.

Sistemas Lineares com Saltos Markovianos (SLSMs) são estudados desde a década de 1960 e vêm ganhando visibilidade desde então, com diversas aplicações dentre as quais Finanças, Robótica e Engenharias diversas. Um problema de regulação trata de controlar o SLSM buscando fazer sua trajetória se aproximar de zero. Quando os saltos markovianos são observados, o problema é simples e bem resolvido, muito diferente de quando não se observam os saltos. Neste trabalho é estudado um algoritmo da literatura utilizado para resolver o problema de regulação sem observação dos saltos, chamado Método Variacional (MV). Sendo um dos melhores métodos para o dado problema, enfrenta dificuldades de cunho numérico. Neste trabalho se procura analisar e melhorar o condicionamento dos subproblemas envolvidos, de forma a favorecer a convergência do método. São testadas abordagens diferentes usando precondicionadores e comparados os resultados, permitindo concluir que três das cinco abordagens é que trouxeram os melhores resultados. Por se tratar de sistemas lineares do tipo $A x=b$, as abordagens de condicionamento podem ser adaptadas para outros problemas semelhantes.

Palavras-chave: Controle ótimo, Sistemas dinâmicos lineares, Cadeias de Markov, Parâmetros com salto, Condicionamento numérico. 



\section{ABSTRACT}

RIBEIRO, J. R. Analysis and improvement of the variational method for control of Markov jump linear systems with no jump observation. 2019. 98 p. Dissertação (Mestrado em Ciências - Ciências de Computação e Matemática Computacional) - Instituto de Ciências Matemáticas e de Computação, Universidade de São Paulo, São Carlos - SP, 2019.

Markov Jump Linear Systems (MJLSs) have been studied since the decade of 1960 and they are gaining visibility ever since, due to a wide range of applications, such as Finance, Robotics, several Engeneerings among others. The so called regulation problem is to control the MJLS seeking to make its trajectory to approach zero. When markovian jumps are observed, the problem is simple and the solution given as closed formulas, which is quite different from the situation when jumps are not observed. We study an algorithm available in literature called Variational Method (VM). Even though it is one of the best methods to solve the problem, it has some numerical difficulties. We analyse its performance and propose some ideas aiming at the ill-conditioning of the subproblems involved, in order to improve the convergence of the method. Different approaches are tested using preconditioners and the results are compared, indicating that three approaches of the five tested ones are promising for convergence improvement. Because the subproblems are linear systems of type $A x=b$, these approaches can be adapted to similar problems.

Keywords: Optimal control, Dynamic linear systems, Markov chains, Jump parameters, Numerical conditioning. 



\section{LISTA DE ILUSTRAÇÕES}

Figura 1 - Ilustração: aplicações de sistemas lineares com saltos markovianos . . . . . 27

Figura 2 - Convergência do Método Variacional. . . . . . . . . . . . . . 27

Figura 3 - Condicionamento dos sistemas $\mathbb{S}_{k}$ do MV em instâncias e iterações diversas. 28

Figura 4 - Esquema de uma $\mathrm{cM} \operatorname{com} N=2 \ldots \ldots \ldots \ldots$

Figura 5 - Duas realizações da $\mathrm{cM}\left\{\mathscr{N}, \mathbb{P}, \pi_{0}\right\} \ldots \ldots \ldots \ldots$

Figura 6 - Grafo representando a cM. . . . . . . . . . . . . 33

Figura 7 - Duas realizações da $\mathrm{cM}\left\{\mathscr{N}, \mathbb{P}, \pi_{0}\right\} \ldots \ldots \ldots \ldots$

Figura 8 - Grafo representando a cM. . . . . . . . . . . . . 34

Figura 9 - Esquema do SLSM: $\theta_{k}$ é aleatório conforme uma cM. . . . . . . . . . 35

Figura 10 - Uma realização da cM e a respectiva trajetória de $x_{k} \ldots \ldots$. . . . . . . . 38

Figura 11 - A trajetória do SLSM para várias simulações da cM e o valor esperado

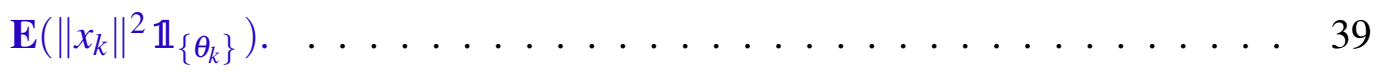

Figura 12 - Uma realização da cM e a respectiva trajetória de $x_{k} \ldots$. . . . . . . . . . 39

Figura 13 - A trajetória do SLSM para várias simulações da cM e o valor esperado $\mathbf{E}\left(\left\|x_{k}\right\|^{2} \mathbb{1}_{\left\{\theta_{k}\right\}}\right) \ldots \ldots \ldots \ldots \ldots \ldots \ldots$. . . . . . . . . . . . . . . . . . . .

Figura 14 - Uma realização da cM e a respectiva trajetória de $x_{k} \ldots \ldots$. . . . . . . . 40

Figura 15 - A trajetória do SLSM para várias simulações da cM e o valor esperado $\mathbf{E}\left(\left\|x_{k}\right\|^{2} \mathbb{1}_{\left\{\theta_{k}\right\}}\right) \ldots \ldots \ldots \ldots \ldots \ldots \ldots$. . . . . . . . . . . . . . . . . . . .

Figura 16 - Uma realização da cM e a respectiva trajetória de $x_{k} \ldots \ldots$. . . . . . . . 41

Figura 17 - A trajetória do SLSM para várias simulações da cM e o valor esperado $\mathbf{E}\left(\left\|x_{k}\right\|^{2} \mathbb{1}_{\left\{\theta_{k}\right\}}\right) \ldots \ldots \ldots \ldots \ldots \ldots \ldots \ldots . \ldots \ldots \ldots$

Figura 18 - A transformação linear deforma o espaço quase em uma reta (exemplo em $\mathbb{R}^{2}$ ). 52

Figura 19 - Comparação dos custos com as máscaras e sem elas, para um total de 1000 instâncias. . . . . . . . . . . . . . . . 66 66

Figura 20 - Comparação dos custos com aproximação quadrática e sem ela. . . . . . . . 66

Figura 21 - Comparação dos custos com uso do P1 e sem ele. . . . . . . . . . . . . . 67

Figura 22 - Comparação dos custos com uso do P2 e sem ele. . . . . . . . . . . . . . 68

Figura 23 - Comparação dos custos com uso do P5 e sem ele. . . . . . . . . . . . . . 68

Figura 24 - Perfis de desempenho dos Precondicionadores. . . . . . . . . . . . . . 69

Figura 25 - Comparação do uso da estratégia de varredura dos valores de $\alpha$. . . . . . . 70

Figura 26 - Perfis de desempenho dos métodos de solução de sistemas lineares com P2. 71

Figura 27 - Perfis de desempenho dos métodos de solução de sistemas lineares com P3. 72

Figura 28 - Perfis de desempenho dos métodos de solução de sistemas lineares com P4. 73 
Figura 29 - Comparação dos custos com as máscaras e sem elas, com P1 . . . . . . . . . 91

Figura 30 - Comparação dos custos com as máscaras e sem elas, com P2 . . . . . . . . . 92

Figura 31 - Comparação dos custos com as máscaras e sem elas, com P3 . . . . . . . . . 92

Figura 32 - Comparação dos custos com as máscaras e sem elas, com P4 . . . . . . . . . 93

Figura 33 - Comparação dos custos com aproximação quadrática e sem ela, com P1. . . 93

Figura 34 - Comparação dos custos com aproximação quadrática e sem ela, com P2. . 94

Figura 35 - Comparação dos custos com aproximação quadrática e sem ela, com P3. . . 94

Figura 36 - Comparação dos custos com aproximação quadrática e sem ela, com P4. . . 95

Figura 37 - Comparação dos custos com uso do P3 e sem ele. . . . . . . . . . . . . . 95

Figura 38 - Comparação dos custos com uso do P4 e sem ele. . . . . . . . . . . . . . . . . . . . . . . . . . . .

Figura 39 - Comparação dos custos com uso de P1 e P2 . . . . . . . . . . . . . . . . . 96

Figura 40 - Comparação dos custos com uso de P2 e P3 . . . . . . . . . . . . . . . 97

Figura 41 - Comparação dos custos com uso de P2 e P4 . . . . . . . . . . . . . . . . . . . . . . 97

Figura 42 - Comparação dos custos com uso de P3 e P4 . . . . . . . . . . . . . . . . 98 


\section{LISTA DE ALGORITMOS}

Algoritmo 1 - Método Variacional . . . . . . . . . . . . . . . . 46

Algoritmo 2 - Operador $\mathscr{T}(\cdot)($ OPERT) . . . . . . . . . . . . . . 48

Algoritmo 3 - Cálculo do custo J (CUSTO) . . . . . . . . . . . . . . . . . . . . . . . . . 48

Algoritmo 4 - A-Ortogonalização de Gram-Schmidt . . . . . . . . . . . . . . . . 90 

Tabela 1 - Exemplo de Perfil de Desempenho. . . . . . . . . . . . . . . . . 62

Tabela 2 - Descrição das etiquetas dos precondicionadores utilizadas no texto. . . . . . 67

Tabela 3 - Descrição das etiquetas dos métodos utilizadas no texto. . . . . . . . . . 70

Tabela 4 - Comparação dos métodos na otimalidade $\left(\right.$ em $\left.10^{0}\right)$, com P2 . . . . . . . 71

Tabela 5 - Comparação dos métodos na otimalidade (em $\left.10^{0}\right)$, com P3 . . . . . . . . 72

Tabela 6 - Comparação dos métodos na otimalidade $\left(\right.$ em $\left.10^{0}\right)$, com P4 . . . . . . . . 73 

$c M$ - Cadeia de Markov

SLSM - Sistema linear com salto markoviano (em inglês MJLS - Markov Jump Linear System)

$F O$ - Função Objetivo

J - Custo da FO

$\mathbb{S}$ - Sistema linear, do tipo $A x=b$

$k$ - Tempo discreto

$\eta$ — Iteração do Método Variacional

$M V$ - Método Variacional

$\mathscr{N}$ — Espaço de estados markoviano (conjunto de índices $i=1, \ldots, N$ )

$N$ - Dimensão do espaço $\mathscr{N}$

$\mathbb{P}$ - Matriz de probabilidade de transição da cM

$\theta_{k}$ - Estado da cM no instante $k$, também chamado estado discreto do sistema dinâmico ou markoviano

' - Transposição de matriz

$\Phi_{\theta}$ - Conjunto de parâmetros do SLSM, indexados em $\mathscr{N}$

$x_{k}$ - Estado contínuo do sistema dinâmico no instante $k$

$R L Q$ - Regulador Linear Quadrático

$\mathscr{H}$ - Horizonte de planejamento (conjunto de índices $k=0, \ldots, T$ )

$\mathbb{V}_{m \times n}^{\mathscr{N}}$ - Espaço vetorial de coleções de matrizes reais $m \times n$.

$T$ - Tamanho do horizonte $\mathscr{H}$

$n$ - Dimensão do estado contínuo $x$

$m$ - Dimensão do controle $u$

$p$ - Dimensão do ruído $\omega$

$\pi_{k}$ - Vetor distribuição de probabilidade de $\theta_{k}$

$\|\cdot\|$ - Norma euclidiana 
$G$ - Matriz de ganho

$\mathbf{E}(\cdot)$ - Operador esperança

$X^{k}$ - Matriz de segundo momento de $x_{k}$

$\mathbb{1}_{\{\mathscr{C}\}}$ - Função indicadora de $\mathscr{C}$

$\overline{\mathbf{A}}, \overline{\mathbf{C}}$ - Matrizes de malha fechada

$\mathscr{T}(\cdot), \mathscr{E}(\cdot), \mathscr{L}(\cdot)$ - Operadores sobre coleções de matrizes

$\Sigma^{k}$ - Matriz de covariância do ruído $\omega$ ponderada por $\pi_{k}$ 
INTRODUÇÃO . . . . . . . . . . . . . . . . . 25

1.1 Motivação e abordagens . . . . . . . . . . . . . 27

$1.2 \quad$ Organização da dissertação . . . . . . . . . . . . . 29

2 SOBRE CADEIAS DE MARKOV E SISTEMAS LINEARES COM SALTOS MARKOVIANOS .................. . . 31

$2.1 \quad$ Cadeias de Markov . . . . . . . . . . . . . 31

$2.2 \quad$ Sistemas Lineares com Saltos Markovianos . . . . . . . . . . . . 34

$2.3 \quad$ Exemplos numéricos $\ldots \ldots \ldots \ldots$

3 MODELO DETERMINÍSTICO E MÉTODO VARIACIONAL . . . 43

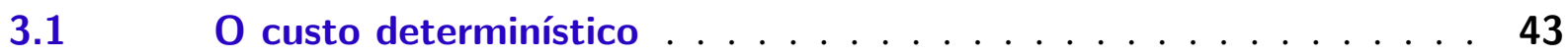

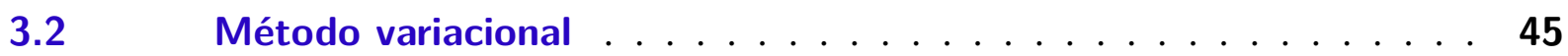

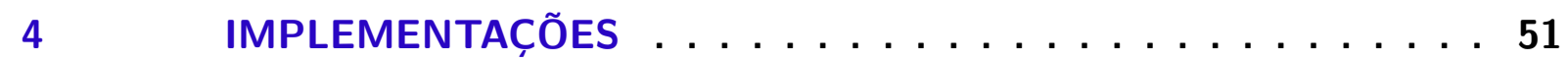

$4.1 \quad$ Mudança de base para os ganhos $G_{k} \ldots \ldots \ldots$

4.2 Mudança de base para os ganhos $G_{k}$ : segunda formulação . . . 55

$4.3 \quad$ Máscaras . . . . . . . . . . . . . . . 58

$4.4 \quad$ Aproximação quadrática . . . . . . . . . . . . 62

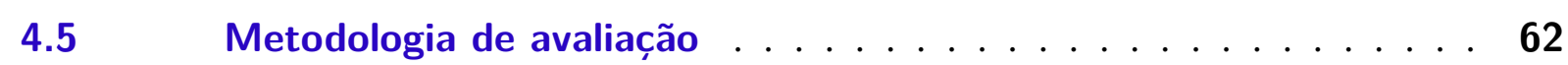

$5 \quad$ RESULTADOS E DISCUSSÕES . . . . . . . . . . . . . 65

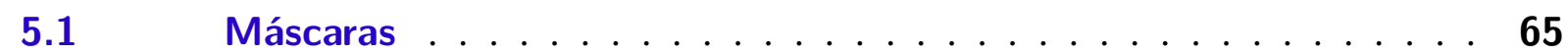

$5.2 \quad$ Aproximação quadrática multivariada . . . . . . . . . . 66

$5.3 \quad$ Precondicionadores . . . . . . . . . . . . . 67

$5.4 \quad$ varredura . . . . . . . . . . . . . . . . . . 69

$5.5 \quad$ Métodos .................... 70

$6 \quad$ CONSIDERAÇÕES FINAIS . . . . . . . . . . . 75

REFERÊNCIAS . . . . . . . . . . . . . . . . . 77

APÊNDICE A CUSTO COMO FUNÇÃO QUADRÁTICA EM $G_{k} \ldots 79$ 
APÊNDICE B APROXIMAÇÃO QUADRÁTICA MULTIVARIADA POR MÍNIMOS QUADRADOS . . . . . . . . . . 85

APÊNDICE C MÉTODO DE DIREÇÕES CONJUGADAS . . . . 89

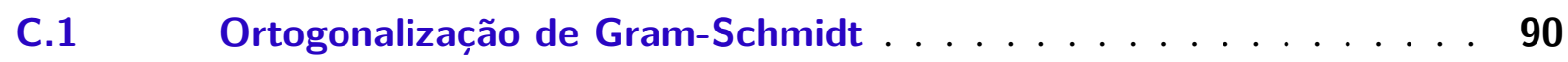

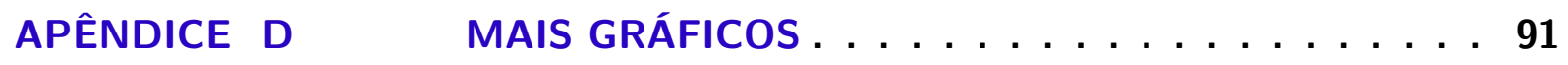

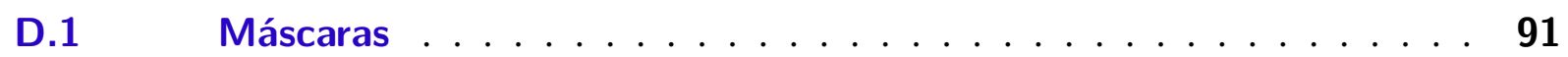

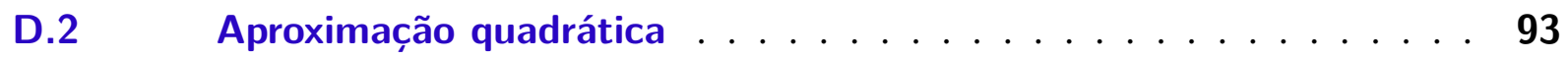

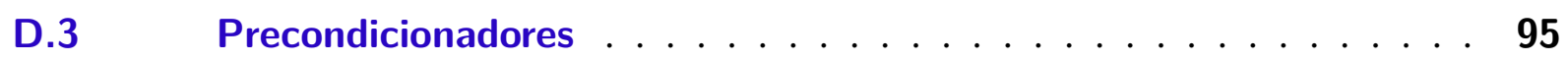




\section{1}

\section{INTRODUÇÃO}

Com o aumento exponencial de tecnologias novas na atualidade, especialmente as eletrônicas, torna-se importante o desenvolvimento de controles para certos objetos, como por exemplo, carros, motores elétricos, aviões, robôs, dentre muitos outros.

No intuito de corrigir a rota de um avião, a trajetória de um robô, a velocidade de trabalho de um motor, dentre outros exemplos, de forma automática, surge a ideia de previsão do sistema e correção de falhas.

Uma classe de sistemas dinâmicos lineares estocásticos que começou a ser estudada desde a década de 1960 com Dynkin (1965) são os sistemas lineares com saltos markovianos (SLSMs). Esses permitem modelar alterações repentinas e bruscas, como em uma pane, falha, ou qualquer outra mudança inesperada dos parâmetros no sistema, o que é comum no mundo real. No momento em que ocorre uma alteração dessas, diz-se ter ocorrido um salto (troca) entre parâmetros do SLSM; markoviano porque o modelo para esses saltos é uma cadeia de Markov (ou cM).

Alguns problemas de controle de SLSMs, como Rastreamento com Alvos Dinâmicos e o Regulador Linear Quadrático, são modelados como problema de otimização, em que algumas restrições são penalizadas, atribuindo-se-lhe um certo custo $\mathbf{J}$ a ser minimizado, dado pela Função Objetivo (FO). Essa penalização e alguns outros fatores tornam o problema não linear, podendo ser resolvido por métodos de descida clássicos da literatura, como os encontrados, por exemplo, em Ribeiro e Karas (2014) e Nocedal e Wright (2006). Entretanto, o problema geralmente é de alta dimensão se tratado com algoritmos de descida, o que induz a utilização de um princípio variacional para quebrar o problema em uma sequência de problemas menores.

Neste trabalho é abordado o problema de regulação de um sistema dinâmico linear com custo quadrático sujeito a saltos markovianos não observados em seus parâmetros. É assumido que apenas o chamado estado contínuo ${ }^{1}$ é observado, problema mais complexo do que

1 Diz-se estado contínuo por tomar valores no espaço $\mathbb{R}^{n}$, enquanto que a outra variável de estado 
aquele em que o estado é completamente observado (ambos os estados contínuo e markoviano, problema cuja solução é dada por uma fórmula fechada, chamada Equações de Riccati Acopladas (ABOU-KANDIL et al., 2003; ABOU-KANDIL; FREILING; JANK, 1995)). Para resolver esses problemas de controle com observação parcial, Val e Başar (1999) desenvolvem um algoritmo iterativo baseado no princípio variacional, em que se resolve um sistema linear $\mathbb{S}_{k}$ do tipo $A x=b$ para cada instante de tempo discreto $k$, o subproblema $\mathbb{S}_{k}$, formando uma sequência de sistemas lineares $\left\{\mathbb{S}_{k}\right\}^{\eta}$ para cada iteração $\eta$ do método.

Algumas variantes desse Método Variacional (MV) foram desenvolvidas, bem como o emprego de algoritmos clássicos de descida, tais como em Vargas (2004) com uma abordagem de horizonte retrocedente aplicada nos problemas de Rastreamento e Regulação com ruído aditivo, Bortolin (2012) trabalhando com o método clássico de Newton Modificado, usando um esquema de observação parcial da cM para o problema de Regulação e desenvolvendo um gerador de instâncias, Silva (2012) com algoritmos evolutivos tipo Genético para o problema de Regulação, e Oliveira (2014) com Gradiente e Newton em uma abordagem de atualização de múltiplos ganhos para o problema de Regulação.

Dentre esses algoritmos propostos na literatura, o Método Variacional dispõe das melhores soluções e tempo computacional de uma forma geral, mas enfrenta alguns obstáculos. Por ser baseado principalmente na resolução de uma sequência de sistemas lineares $\mathbb{S}_{k}$, volta-se a atenção ao condicionamento das matrizes desses sistemas $\mathbb{S}_{k}$ e para os métodos de solução dos mesmos. Nenhum estudo da literatura dá atenção a esse aspecto, o que levanta algumas questões: o condicionamento é realmente um problema, ou as matrizes são em geral bem condicionadas? O método de solução de $\mathbb{S}_{k}$ é eficiente ou deixa um grande resíduo? Se empregado um método iterativo, ele converge ou precisa de uma modificação? Dado um erro na solução de um desses $\mathbb{S}_{k}$, o que acontece com o valor $\mathbf{J}$ da FO?

Algumas destas questões são respondidas já nos testes computacionais preliminares, confirmando a problemática e voltando a atenção às abordagens para resolvê-la. Assim, busca-se melhorar o desempenho do Método Variacional, bem como tentar responder essas questões.

Os SLSMs podem se aplicados em diversas áreas, como por exemlo em Finanças (CAJUEIRO, 2002; VAL; BAŞAR, 1999), Robótica (VARGAS; COSTA; VAL, 2013), Engenharia Aeroespacial (STOICA; YAESH, 2002), Sistemas de Comunicação (ABDOLLAHI, 2008; ABDOLLAHI; KHORASANI, 2011), dentre várias outras.

(variável de salto) toma valores em $\mathbb{N}$, sendo chamada de estado discreto ou markoviano. 
Figura 1 - Ilustração: aplicações de sistemas lineares com saltos markovianos

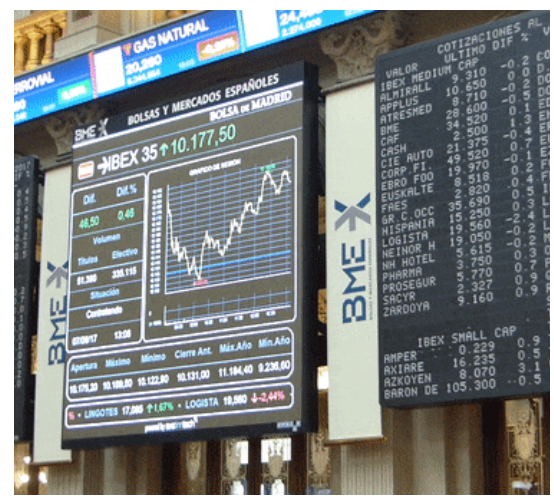

(a) Finanças

Fonte: González (2017).

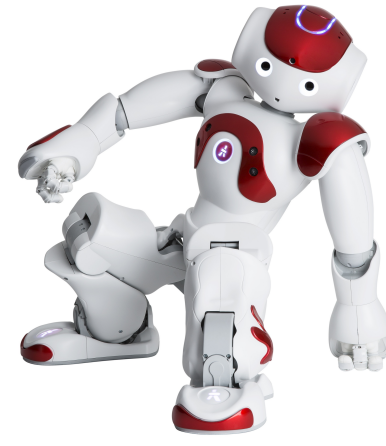

(b) Robótica

Fonte: Europe (2017).

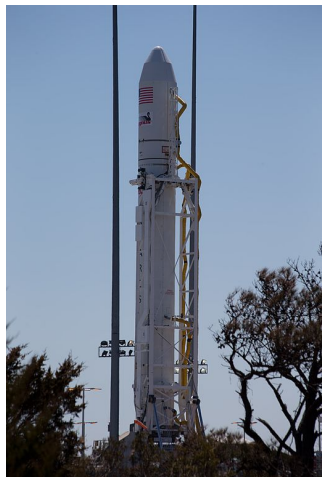

(c) Engenharia aeroespacial

Fonte: NASA (2016).

\subsection{Motivação e abordagens}

O próximo gráfico ilustra o comportamento geral do valor da FO (custo $\mathbf{J}$ ) versus o número de iterações do MV. Nem sempre se consegue o comportamento esperado do decaimento do custo ao longo das iterações, conforme provado matematicamente em Val e Başar (1999), por vezes ferindo a monotonicidade, como se pode ver na Figura 2.

Figura 2 - Convergência do Método Variacional.
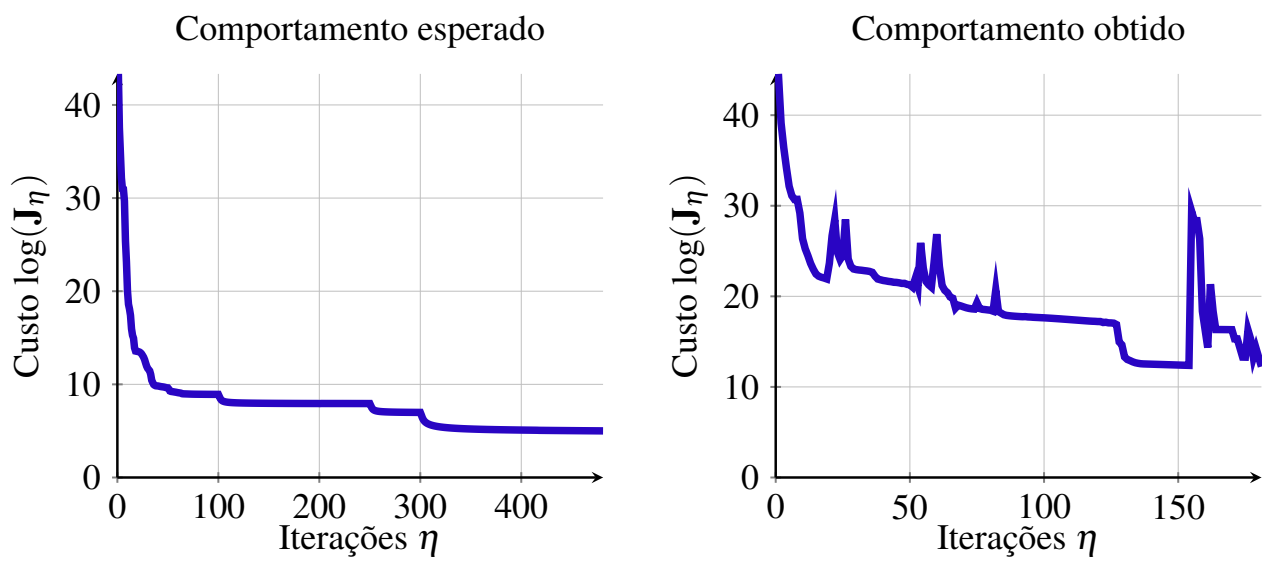

Observação 1. Como visto no gráfico da convergência (Figura 2), por não se ter um resultado matemático que garanta otimalidade da solução, não há um critério de parada para o MV que seja completamente satisfatório. Assim, se o leitor for implementar o controle ótimo em um dispositivo físico (Arduino por exemplo), é importante deixar o MV executando por um bom tempo, pois mesmo supondo que o algoritmo convirja conforme o gráfico do comportamento

Figura 1a: GONZÁLEZ, B. N. Palacio de la Bolsa de Madrid. 2017. Licença (C) CC BY-SA 4.0.

Figura 1b: EUROPE, S. R. NAO Robot. 2017. Licença (C) CC BY-SA 4.0.

Figura 1c: NASA. NASA Goddard Space Flight Center. 2016. Licença (C) CC BY 2.0. 
esperado (vide a referida figura), pode produzir alguns "falsos positivos", isto é, parece que o algoritmo convergiu e pára de diminuir substancialmente o custo. No entanto, repentinamente o custo volta a decrescer bastante, e isso se repete por diversas vezes (atente-se para as iterações $\eta=100, \eta=250$ e $\eta=300$ do gráfico mencionado). Por esse motivo, o critério de parada por custo relativo é válido, mas não muito eficiente. Ainda assim, não é fácil estabelecer um critério de parada satisfatório, pois vai depender de cada caso.

Procurando investigar se o número de condição dos sistemas lineares $\mathbb{S}_{k}$ são um problema a ser tratado, verificou-se que os mesmos são razoavelmente bem condicionados na maioria dos casos, mas que ainda há uma grande quantidade de sistemas lineares mal condicionados, conforme se vê no próximo gráfico. Nele foram colocados os números de condição dos sistemas lineares $\mathbb{S}_{k}$ de diversas instâncias escolhidas ao acaso, em iterações também diversificadas, contabilizando um total de 5000 sistemas lineares analisados. Na Figura 3 pode-se ver que muitas vezes o condicionamento numérico do sistema $\mathbb{S}_{k}$ é muito grande, e com isso as soluções obtidas para esses subproblemas não são boas, prejudicando a convergência. Em alguns testes particulares realizados, o condicionamento numérico de até $10^{6}$ é razoavelmente aceitável para o Matlab (pacote utilizado para implementação do algoritmo), com uma precisão de aproximadamente 10 dígitos corretos. Na figura, a linha vermelha indica $10^{6}$.

Figura 3 - Condicionamento dos sistemas $\mathbb{S}_{k}$ do MV em instâncias e iterações diversas.

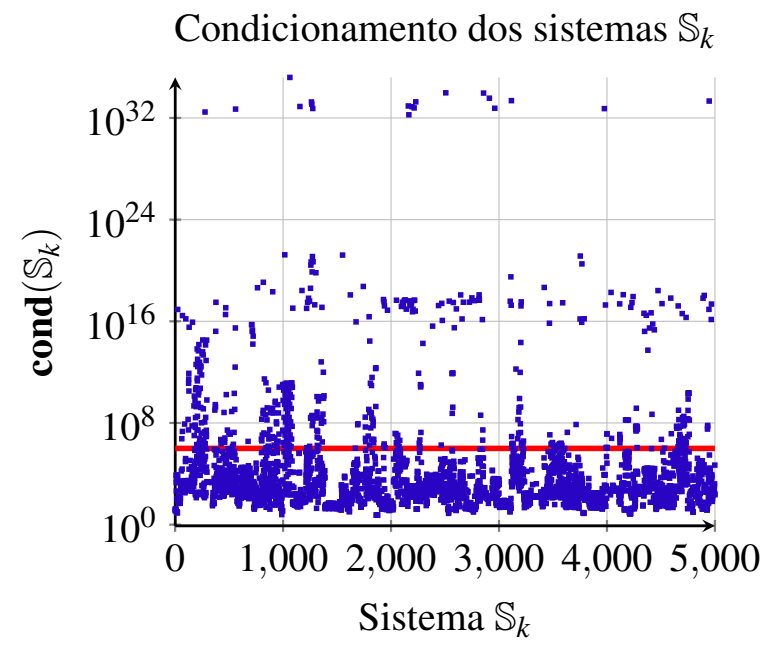

Algumas abordagens para tratar o condicionamento dos sistemas $\mathbb{S}_{k}$ foram testadas, sendo a mais proveitosa delas o uso de precondicionadores para os $\mathbb{S}_{k}$, baseados na decomposição de valores singulares ou SVD (Singular Value Decomposition), por meio de uma mudança de base. Ainda que teoricamente equivalentes, algumas variações dessa implementação dos precondicionadores tiveram resultados bem diferentes, apenas trocando a prioridade de operadores lineares. Isso instigou a procura de formulações distintas para a mudança de base envolvida, levando aos resultados da Seção 4.1 e da Seção 4.2. Uma modificação foi feita com a adição de um parâmetro $\alpha$ permitindo a criação de diferentes bases para o precondicionamento. 
Outra metodologia aplicada foi a modelagem dos parâmetros que constituem $\mathbb{S}_{k}$ por meio de Aproximação Quadrática Multivariada por Mínimos Quadrados, (cuja formulação foi escrita no Apêndice B). Mais uma abordagem foi a utilização de um tipo de máscara em um dos parâmetros do modelo com a função de minimizar a perda numérica de uma certa estrutura dos espaços vetoriais ao longo das iterações.

\subsection{Organização da dissertação}

No Capítulo 2 é apresentada uma introdução às cM's com dois exemplos numéricos, que pode ser omitida, caso o leitor já tenha familiaridade, e em seguida o modelo do SLSM para o problema de Regulação com horizonte finito, objeto deste trabalho. Quatro exemplos numéricos são dados para ilustrar o comportamento dos SLSMs sem controle e com controle, sem ruído aditivo e com ruído aditivo.

No Capítulo 3 é apresentado o modelo determinístico e algumas definições importantes, para então apresentar o Método Variacional e um esquema de seu funcionamento. No Capítulo 4 fazem-se as discussões das implementações e por fim, no Capítulo 5 os resultados obtidos. Alguns resultados foram colocados nos apêndices, por serem extensos e servirem mais como demonstração ou apresentação dos fatos utilizados. Ao final são apresentadas algumas considerações e futuros trabalhos no Capítulo 6. 

CAPÍTULO

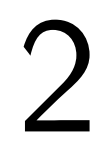

\section{SOBRE CADEIAS DE MARKOV E SISTEMAS LINEARES COM SALTOS MARKOVIANOS}

No intuito de contextualizar ao leitor a respeito do que são cadeias de Markov e a seguir sobre Sistemas Lineares com Saltos Markovianos (SLSMs), faz-se uma introdução ao assunto neste capítulo, com uma seção de exemplos em seguida.

\subsection{Cadeias de Markov}

Uma cadeia de Markov (cM) em tempo discreto é uma sequência de variáveis aleatórias $\left\{\theta_{k}\right\}_{k \in \mathbb{N}}$, que toma valores em um conjunto de índices chamado de espaço de estados $\mathscr{N}=$ $\{1,2, \ldots, N\}$, com uma matriz estocástica contendo as probabilidades da transição entre esses estados $\mathbb{P} \in[0,1]^{N \times N}$. Também pode ser atribuída uma distribuição de probabilidade inicial $\pi_{0} \in[0,1]^{N \times 1}$ para o primeiro "sorteio" da cM (valor que o primeiro estado da cadeia $\theta_{0}$ assume). Assim, essa cadeia pode ser representada por $\left\{\mathscr{N}, \mathbb{P}, \pi_{0}\right\}$.

Em cada instante de tempo $k$, o estado da cadeia assume aleatoriamente um valor do espaço de estados $\mathscr{N}$ com alguma probabilidade dependente apenas do estado imediatamente anterior $k-1$. Na matriz de transição se situam as probabilidades condicionais de mudança de estado. Veja o exemplo na Figura 4, o esquema de uma cM com dois estados, ou seja, $N=2$.

Considere $\operatorname{Prob}(\cdot)$ uma medida de probabilidade no espaço fundamental. Na Figura 4, a probabilidade condicional de que $\theta_{k}=2$ dado que $\theta_{k-1}=1$ é dada por $\mathbb{P}_{12}$, ou seja, $\operatorname{Prob}\left(\theta_{k}=\right.$ $\left.2 \mid \theta_{k-1}=1\right)=\mathbb{P}_{12}$. Da mesma forma, a probabilidade condicional de que $\theta_{k}=1$ dado que $\theta_{k-1}=1$ é dada por $\mathbb{P}_{11}$, ou seja, $\operatorname{Prob}\left(\theta_{k}=1 \mid \theta_{k-1}=1\right)=\mathbb{P}_{11}$ e assim por diante. De uma forma geral, tem-se a matriz de probabilidade de transição definida como sendo:

$$
\mathbb{P}=\left[\mathbb{P}_{i j}\right]=\operatorname{Prob}\left(\theta_{k}=j \mid \theta_{k-1}=i\right)
$$


Figura 4 - Esquema de uma cM $\operatorname{com} N=2$.

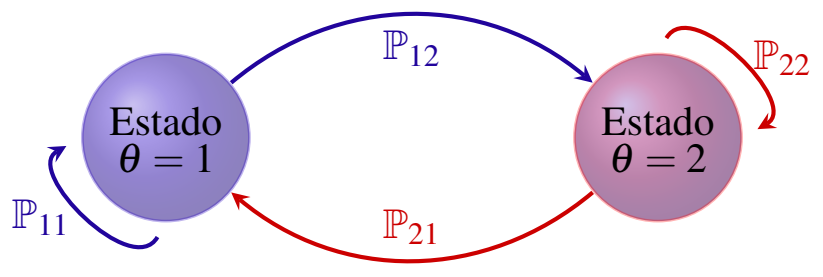

Fonte: Elaborada pelo autor.

Um estado da cM é dito transiente ou temporário quando após uma finita quantidade de vezes que este estado é "sorteado", ele não será mais sorteado. Formalmente, se a partir de um estado $j$ existe uma probabilidade estritamente positiva de que a cadeia não visite nunca mais esse estado, então ele é dito transiente. Um estado da cM que não é transiente é dito recorrente, ou seja, se a cadeia passar por esse estado alguma vez, então ele será revisitado uma quantidade infinita de vezes.

Uma propriedade muito útil advinda do teorema da probabilidade total é que, dada uma distribuição de probabilidade $\pi_{k}$ dos estados da $\mathrm{cM}$, para conhecer a distribuição $\pi_{k+1}$, basta multiplicá-lo pela matriz $\mathbb{P}^{\prime}$, em que $\mathbb{P}^{\prime}$ indica a matriz transposta de $\mathbb{P}$, formando a recursão

$$
\pi_{k+1}=\mathbb{P}^{\prime} \pi_{k}
$$

Exemplo 1. Considerando o espaço de estados markoviano $\mathscr{N}=\{1,2,3\}$, uma distribuição de probabilidade inicial $\pi_{0}=(1 / 2,1 / 16,7 / 16)^{\prime}$ e uma matriz de probabilidade de transição $\mathbb{P}$ definida abaixo, pode-se obter várias de suas propriedades, calcular as distribuições $\pi_{k}$ para qualquer instante $k$ e também simular a cadeia $\left\{\mathscr{N}, \mathbb{P}, \pi_{0}\right\}$.

$$
\mathbb{P}=\begin{array}{cccc}
\text { Estados } & \theta_{k+1}=1 & \theta_{k+1}=2 & \theta_{k+1}=3 \\
\theta_{k}=1 \\
\theta_{k}=2 \\
\theta_{k}=3
\end{array}\left[\begin{array}{ccc}
0.9 & 0 & 0.1 \\
0 & 0 & 1 \\
0 & 0.9 & 0.1
\end{array}\right]
$$

Tem-se pela Equação 2.2 que $\pi_{1} \approx(0.4500,0.3937,0.1562)^{\prime}, \pi_{2} \approx(0.4050,0.1406,0.4543)^{\prime}$ e $\pi_{\infty} \approx \pi_{10^{4}} \approx(0,0.4736,0.5263)^{\prime}$.

$$
\mathbb{P}^{10} \approx\left[\begin{array}{ccc}
0.3486 & 0.3085 & 0.3428 \\
0 & 0.6571 & 0.3428 \\
0 & 0.3085 & 0.6914
\end{array}\right] \quad \mathbb{P}^{\infty} \approx \mathbb{P}^{10^{4}} \approx\left[\begin{array}{ccc}
0 & 0.4736 & 0.5263 \\
0 & 0.4736 & 0.5263 \\
0 & 0.4736 & 0.5263
\end{array}\right]
$$

Pela matriz $\mathbb{P}$, pode-se ver que a probabilidade de que o estado se mantenha em 1 é de $90 \%$, em 2 é zero ( $\operatorname{se} \theta_{k}=2$, então $\theta_{k+1} \neq 2$, qualquer que seja $k$ ) e em 3 é $10 \%$. Ao observar a matriz $\mathbb{P}^{\infty}$, nota-se que o estado 1 é transiente e os outros são recorrentes. Ademais, tem-se que, no limite (vide $\pi_{\infty}$ ), a probabilidade de que a cadeia sorteie o estado 2 é $\operatorname{Prob}\left(\theta_{k}=2 \mid \theta_{k-1}\right) \approx 0.4736$, enquanto que a probabilidade de sortear o estado 3 é $\operatorname{Prob}\left(\theta_{k}=3 \mid \theta_{k-1}\right) \approx 0.5263$. 
No gráfico abaixo constam os sorteios realizados da cadeia de $k=1$ até $k=200$.

Figura 5 - Duas realizações da $\mathrm{cM}\left\{\mathscr{N}, \mathbb{P}, \pi_{0}\right\}$.
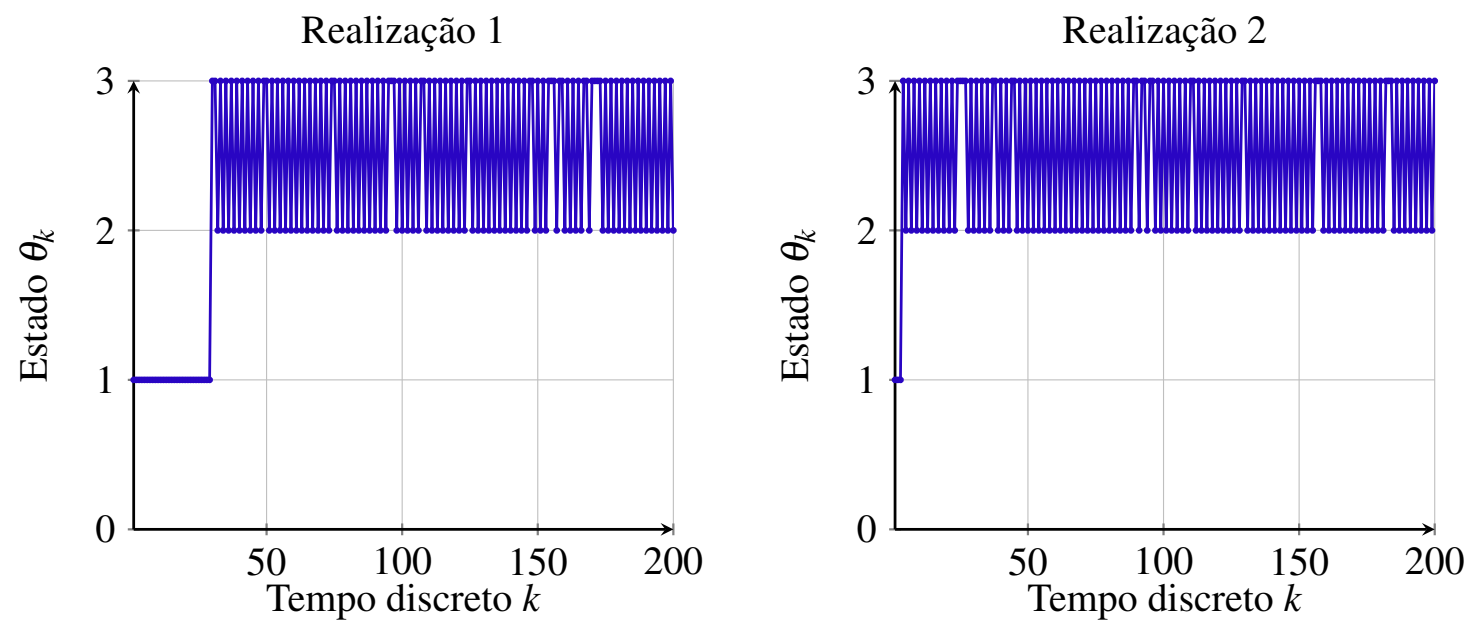

Figura 6 - Grafo representando a cM.

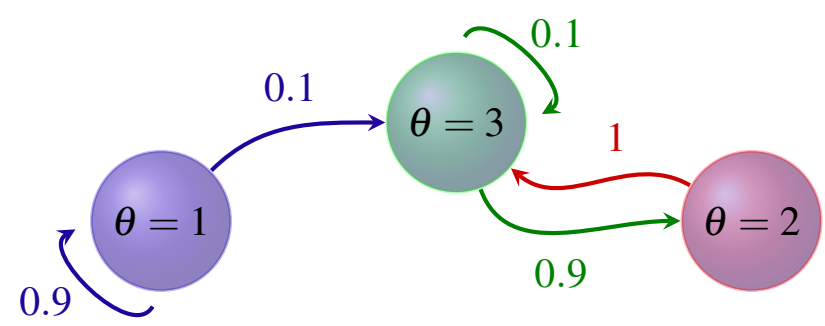

Fonte: Elaborada pelo autor.

Exemplo 2. Considerando o espaço de estados markoviano $\mathscr{N}=\{1,2\}$, uma distribuição de probabilidade inicial $\pi_{0}=(0.98,0.02)^{\prime}$ e uma matriz de probabilidade de transição $\mathbb{P}$ definida abaixo, pode-se obter várias de suas propriedades, calcular as distribuições $\pi_{k}$ para qualquer instante $k$ e também simular a cadeia $\left\{\mathscr{N}, \mathbb{P}, \pi_{0}\right\}$.

$$
\mathbb{P}=\begin{aligned}
& \text { Estados } \\
& \theta_{k}=1 \\
& \theta_{k}=2
\end{aligned} \quad\left[\begin{array}{cc}
\theta_{k+1}=1 & \theta_{k+1}=2 \\
0.92 & 0.08 \\
0.01 & 0.99
\end{array}\right]
$$

Tem-se pela Equação 2.2 que $\pi_{1}=(0.9018,0.0982)^{\prime}, \pi_{2} \approx(0.8306,0.1693)^{\prime}$ e $\pi_{\infty} \approx \pi_{10^{4}} \approx$ $(0.1111,0.8888)^{\prime}$.

$$
\mathbb{P}^{10} \approx\left[\begin{array}{ll}
0.4572 & 0.5427 \\
0.0678 & 0.9321
\end{array}\right] \quad \mathbb{P}^{\infty} \approx \mathbb{P}^{10^{4}} \approx\left[\begin{array}{ll}
0.1111 & 0.8888 \\
0.1111 & 0.8888
\end{array}\right]
$$

Nota-se que ambos os estados são recorrentes, veja $\mathbb{P}^{\infty}$. Ademais, tem-se que, no limite (vide $\pi_{\infty}$ ), há uma grande probabilidade de que a cadeia sorteie o estado 2, pois, como se vê, $\operatorname{Prob}\left(\theta_{k}=\right.$ $\left.2 \mid \theta_{k-1}\right) \approx 8 / 9$, enquanto que a probabilidade de sortear o estado 1 é $\operatorname{Prob}\left(\theta_{k}=1 \mid \theta_{k-1}\right) \approx 1 / 9$. 
No gráfico abaixo constam os sorteios realizados da cadeia de $k=1$ até $k=200$.

Figura 7 - Duas realizações da $\mathrm{cM}\left\{\mathscr{N}, \mathbb{P}, \pi_{0}\right\}$.
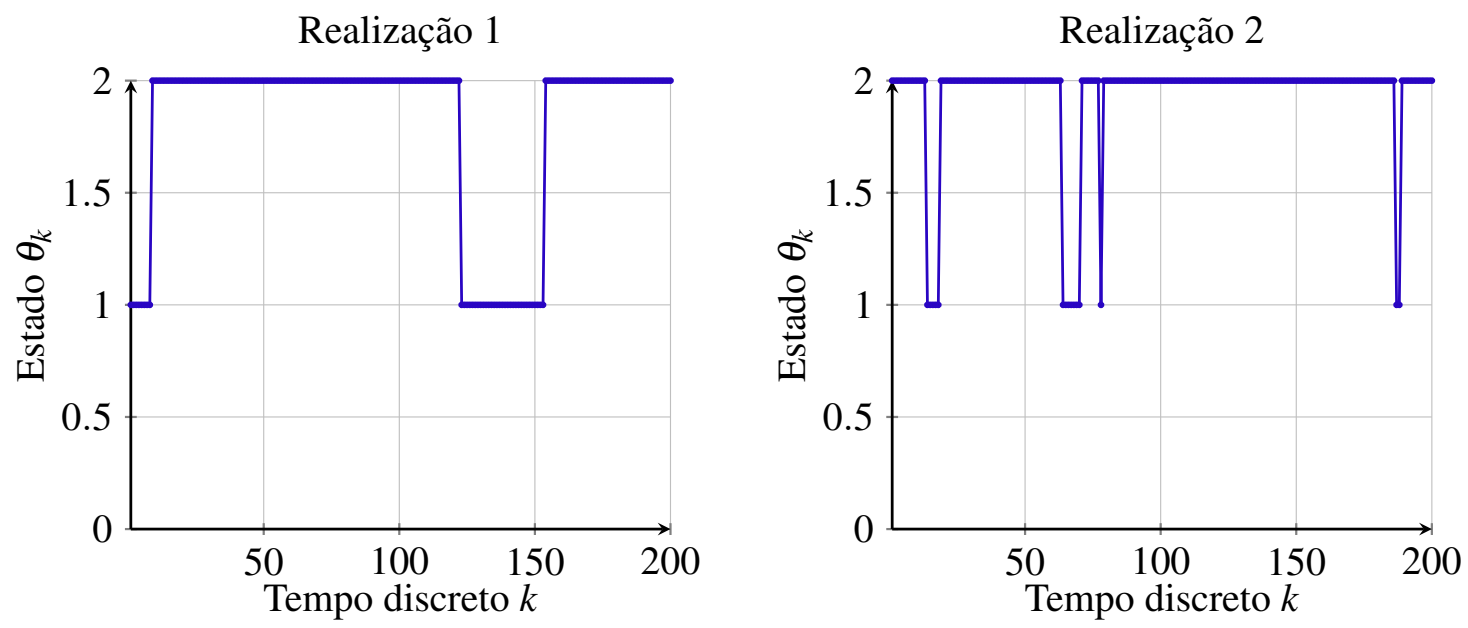

Figura 8 - Grafo representando a cM.

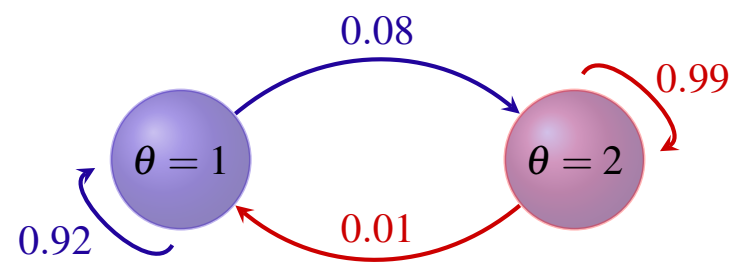

Fonte: Elaborada pelo autor.

Uma propriedade importante das cM's, chamada de Propriedade de Markov, é que elas possuem dependência apenas na vizinhança, ou seja, as cadeias não dependem de todo o histórico, mas apenas do instante anterior:

$$
\operatorname{Prob}\left(\theta_{k} \mid \theta_{k-1}, \theta_{k-2}, \ldots, \theta_{0}\right)=\operatorname{Prob}\left(\theta_{k} \mid \theta_{k-1}\right)
$$

\subsection{Sistemas Lineares com Saltos Markovianos}

Os chamados Sistemas Lineares com Saltos Markovianos (SLSMs) foram introduzidos por Dynkin (1965) e têm sido usado para modelar sistemas dinâmicos lineares que mudam de comportamento bruscamente (salto). Uma cM é utilizada para modelar os saltos nos parâmetros, de modo que, num instante discreto $k$ em que a cadeia esteja no estado $\theta_{k}$, o SLSM utiliza um conjunto de parâmetros $\Phi_{\theta_{k}}$. Os exemplos numéricos que virão mais adiante ilustram a ideia de um SLSM.

O estado de um SLSM possui dois componentes: um estado contínuo em $\mathbb{R}^{n}$ que representa a variável do sistema dinâmico (denotado pelo vetor $x_{k}$ ) e um estado discreto em 
$\mathscr{N}$ representando a cM (denotado por $\theta_{k}$ ), de modo que a cada instante de tempo discreto $k$, o estado completo do sistema é $\left(x_{k}, \theta_{k}\right)$. O vetor $x$ pode representar, por exemplo, grandezas físicas do sistema real como posição, velocidade, aceleração, corrente elétrica, etc., os quais variam no tempo de acordo com um modelo linear. Os $\theta_{k}$ representam o modo de operação do sistema dinâmico, como por exemplo, modo "normal", "falha", "fácil", "difícil", "compra", "venda", etc., para os quais os parâmetros $\Phi_{\theta}$ precisam refletir essas nomenclaturas. Veja o esquema da Figura 9.

Figura 9 - Esquema do SLSM: $\theta_{k}$ é aleatório conforme uma cM.

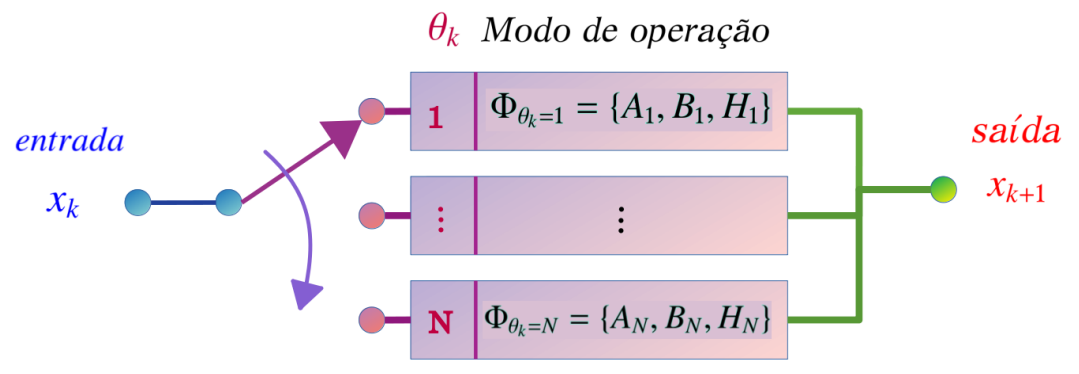

Fonte: Elaborada pelo autor.

Em se tratando dos SLSMs, existem dois problemas de controle importantes: o problema de Rastreamento de Alvos Dinâmicos, cujo objetivo é controlar o sistema de modo que o estado contínuo $x_{k}$ se aproxime de uma trajetória $\left\{\bar{x}_{k}\right\}_{k \in \mathscr{H}}$ previamente definida. Outro problema, chamado de Problema de Regulação ou Regulador Linear Quadrático (RLQ), é um caso particular do primeiro, em que a trajetória desejada é a origem $\left(\bar{x}_{k}=0 \forall k \in \mathscr{H}\right)$. Vargas (2004) e Val e Başar (1999) estudam os dois casos.

Quando o valor de $x_{k}$ é lido do sistema do mundo real através de sensores, bem como cada um dos $\theta_{k}$, diz-se que o SLSM tem o estado completamente observado, $\left(x_{k}, \theta_{k}\right)$. Se isso acontece, o controle ótimo para esse SLSM é bem conhecido na literatura, tem solução direta por meio de Equações de Riccati Acopladas. Entretanto, quando não se tem acesso à cM ( $\theta_{k}$ é totalmente desconhecido, exceto pela sua distribuição inicial $\pi_{0}$ ), diz-se que o SLSM tem o estado parcialmente observado, $\left(x_{k}\right)$, e a abordagem anterior já não funciona. Nesse cenário, o método iterativo baseado em procedimento variacional proposto por Val e Başar (1999) e incrementado por Vargas (2004) vem sendo empregado.

Nem sempre é possível e às vezes é pouco viável mensurar o valor de $\theta$ em muitas aplicações reais, de modo que seria interessante obter uma forma de calcular o controle para esse cenário com estado parcialmente observado ( $\theta$ desconhecido). Já existem na literatura trabalhos e métodos para resolver esse tipo de problema com algumas variações de cenário ou abordagem, como se pode ver, por exemplo, em Val e Başar (1999), Vargas (2004), Bortolin (2012), Silva (2012), Oliveira (2014). Dentre todos estes, o Método Variacional vem se mostrando um dos melhores em termos de tempo e qualidade de solução (custo). 
Definição 1 (Espaço vetorial $\mathbb{V}_{m \times n}^{\mathcal{N}}$ ). Defina o seguinte espaço vetorial de coleções de matrizes: $\mathbb{V}_{m \times n}^{\mathcal{N}}=\left\{U_{i} ; U_{i} \in \mathbb{R}^{m \times n} \forall i \in \mathscr{N}=\{1, \ldots, N\}\right\}$. Caso as matrizes sejam quadradas, faça $m=n$. Considere também o operador traço de matriz $\operatorname{tr}(\cdot)$. Será interessante definir uma multiplicação entre elementos de espaços distintos: •: $\left(\mathbb{V}_{n \times m}^{\mathcal{N}}, \mathbb{R}^{m \times n}\right) \mapsto \mathbb{V}_{n \times n}^{\mathcal{N}}$ ou $\bullet:\left(\mathbb{R}^{m \times n}, \mathbb{V}_{n \times m}^{\mathcal{N}}\right) \mapsto \mathbb{V}_{m \times m}^{\mathcal{N}}$. Assim, defina a soma $X+Y$, produto $X \bullet Y$ e produto interno $\langle X, Y\rangle$ entre pontos desse espaço vetorial conforme

$$
\begin{cases}{[X+Y]_{i}=X_{i}+Y_{i},} & \forall i \in \mathscr{N}, X, Y \in \mathbb{V}_{m \times n}^{\mathscr{N}} \text { (soma), } \\ {[X Y]_{i}=X_{i} Y_{i},} & \forall i \in \mathscr{N}, X \in \mathbb{V}_{m \times n}^{\mathscr{N}}, Y \in \mathbb{V}_{n \times m}^{\mathscr{N}} \text { (produto), } \\ {[B \bullet G]_{i}=B_{i} G,} & \forall i \in \mathscr{N}, B \in \mathbb{V}_{n \times m}^{\mathscr{N}}, G \in \mathbb{R}^{m \times n} \text { (produto), } \\ \langle X, Y\rangle=\sum_{i \in \mathscr{N}} \operatorname{tr}\left(X_{i}^{\prime} Y_{i}\right), & X, Y \in \mathbb{V}_{m \times n}^{\mathscr{N}} \text { (produto interno). }\end{cases}
$$

Simplificando, as operações são feitas matriz por matriz, cada uma com seu índice correspondente $i \in \mathscr{N}$. No caso de $\bullet$, é como se fossem duas coleções sendo multiplicadas matriz a matriz, mas que uma dessas coleções tem todas as matrizes repetidas; no exemplo $B \bullet G, G$ é como se fosse uma coleção de matrizes repetidas. Assim, ainda que essa notação não seja usual, é intuitiva, bastando observar os espaços das variáveis.

Observação 2 (Abusos de notação sobre coleções de matrizes). No intuito de esclarecer a notação utilizada a seguir, fazem-se algumas explicações dos abusos de notação. Inicialmente, defina o conjunto $\mathscr{N}=\{1,2\}$ para exemplificar. Tenha o leitor em mente de que serão utilizadas coleções de matrizes, como por exemplo $A \in \mathbb{V}_{n \times n}^{\mathscr{N}}$, de modo que, quando se tratar de uma matriz específica dessa coleção, indica-se um índice como em $A_{i}$. Exemplo: $A=\left\{A_{1}, A_{2}\right\}$, mas quando se tratar de uma dessas matrizes especificamente, atribui-se-lhe um índice $i \in \mathscr{N}$, de modo que $A$ representa toda a coleção e $A_{2}$ representa a segunda matriz da coleção. A notação será análoga para outras coleções de matrizes, como $B, H, C$ e $D$. Essa notação com apenas um índice é usada para coleções invariantes no tempo, sendo pontos de $\mathbb{V}_{m \times n}^{\mathcal{N}}$ para $m$ e $n$ adequados.

Existem algumas coleções de matrizes no modelo que formam uma sequência/trajetória no tempo, e por isso insere-se mais um índice $k \in \mathscr{H}=\{0,1,2,3\}$ (por exemplo) para especificar qual o instante de tempo a que se refere, além do índice markoviano $i \in \mathscr{N}$, como por exemplo $X=\left\{X_{i}^{k}\right\}_{i \in \mathscr{N}}^{k \in \mathscr{H}}=\left\{\left\{X_{i}^{0}\right\},\left\{X_{i}^{1}\right\},\left\{X_{i}^{2}\right\},\left\{X_{i}^{3}\right\}\right\}_{i \in \mathscr{N}}$, em que $X$ é toda a trajetória de coleções, $X^{k} \in \mathbb{V}_{n \times n}^{\mathcal{N}}$ é a coleção no instante $k, X_{i}^{k} \in \mathbb{R}^{n \times n}$ é a matriz $i$ da coleção $X^{k}$. Sendo assim, $X^{2}$ denota a coleção de matrizes $X^{2}=\left\{X_{1}^{2}, X_{2}^{2}\right\}$. A notação será análoga para outras matrizes, como $L, \Sigma, \sigma, \overline{\mathbf{A}}, \overline{\mathbf{C}}$ e $\pi$. Essa notação com os índices $k \in \mathscr{H}$ será então usada para coleções dinâmicas no tempo.

Pensando em um algoritmo iterativo, que é o caso do MV, ainda pode-se adicionar mais um índice $\eta$ indicando a iteração do método, de modo que, por exemplo, $X$ seja um array pentadimensional, pois tem dimensão $n \times n \times N \times T \times$ maxIt, em que maxIt é o número

De modo que $A$ é um array tridimensional e $A_{i}$ é um array bidimensional.

De modo que $X$ é um array quadridimensional, $X^{k}$ é um array tridimensional e $X_{i}^{k}$ é bidimensional. 
máximo de iterações do MV. Por outro lado, algumas matrizes do modelo não têm componente markoviana, que é o caso das matrizes de ganho $G$, ainda que sejam dinâmicas no tempo. Assim, os índices de $G$ são apenas o tempo $k$ e a iteração $\eta$.

É dada abaixo a definição de um modelo discreto para um RLQ com horizonte de planejamento finito $\mathscr{H}=\{0,1, \ldots, T\}$, objeto de estudo desta dissertação.

Definição 2 (Modelo RLQ). Considere $x_{k} \in \mathbb{R}^{n}, u_{k} \in \mathbb{R}^{m}$ e $\omega_{k} \in \mathbb{R}^{p}$, em que $\omega_{k}$ são vetores aleatórios i.i.d. independentes das demais variáveis do sistema dinâmico, de média zero e matriz de covariância igual à identidade. Considere ainda a cadeia de $\operatorname{Markov}\left\{\mathscr{N}, \mathbb{P}, \pi_{0}\right\}$ que modela os saltos dos parâmetros $\Phi_{\theta}$, com $\mathscr{N}=\{1, \ldots, N\}$. Além disso, sejam as matrizes $A, C \in \mathbb{V}_{n \times n}^{\mathcal{N}}, B \in \mathbb{V}_{n \times m}^{\mathscr{N}}, H \in \mathbb{V}_{n \times p}^{\mathcal{N}}$ e $D \in \mathbb{V}_{m \times m}^{\mathscr{N}}$, de modo a formar os conjuntos de parâmetros $\Phi_{\theta}=\left\{A_{\theta}, B_{\theta}, H_{\theta}, C_{\theta}, D_{\theta}\right\}$ em que $C_{i} \geq 0$ (positiva semidefinida) e $D_{i}>0$ (positiva definida) para cada $i \in \mathscr{N}$. Assim, para cada $k \in \mathscr{H}$, o modelo do RLQ é

$$
\mathbb{H}_{0}\left\{\begin{array}{l}
x_{k+1}=A_{\theta_{k}} x_{k}+B_{\theta_{k}} u_{k}+H_{\theta_{k}} \omega_{k}, \\
z_{k}=x_{k}^{\prime} C_{\theta_{k}} x_{k}+u_{k}^{\prime} D_{\theta_{k}} u_{k}, \\
x_{0} \text { dado, } \theta_{0} \sim \pi_{0}, \mathbb{P}, \theta_{k} \in \mathscr{N}, k \in \mathscr{H},
\end{array}\right.
$$

em que $u_{k}$ representa o controle aplicado ao sistema dinâmico. A saída $z_{k}$ é um custo associado à penalização da norma de $x_{k}$ e da respectiva ação de controle $u_{k}$ e as matrizes simétricas $C_{i}=C_{i}^{\prime} \mathrm{e}$ $D_{i}=D_{i}^{\prime}$ são os pesos da penalização, exigindo-se portanto a positividade de $C_{i}$ e $D_{i}$ para todo $i \in \mathscr{N}$. Exige-se $C \geq 0$ para que o custo de $\left\|x_{k}\right\|$ não seja negativo, e $D>0$ para que, além de não permitir custo negativo para $\left\|u_{k}\right\|$, não permita que $\left\|u_{k}\right\| \rightarrow$ com custo zero (controle infinito sem custo). A matriz de probabilidade de transição da cadeia de Markov é dada pela matriz $\mathbb{P} \in[0,1]^{N \times N}$ conforme descrito na Equação 2.1, e contém probabilidades condicionais com relação a $\theta_{k}$. O vetor $\pi_{0} \in[0,1]^{N}$ é a distribuição de probabilidade de $\theta_{0}$. Essa distribuição $\pi_{k}$ é facilmente calculada para qualquer instante de tempo pela Equação 2.2. Desse modelo, os parâmetros $\left\{\mathscr{N}, \mathbb{P}, \pi_{0}\right\}$ da cM e $\Phi_{\theta}$ para todo $\theta \in \mathscr{N}$ do SLSM são todos conhecidos a priori.

Uma vez que não se tem acesso à informação de $\theta_{k}$, o controle deve se basear apenas no conhecimento de $x_{k}$ e considerar a distribuição de probabilidade de $\theta_{k}$, que é facilmente calculada pela Equação 2.2. Para o modelo $\mathbb{H}_{0}$, a equação de realimentação do controle pode ser escrita como uma função linear do estado contínuo, o que simplifica a implementação. Se o modelo $\mathbb{H}_{0}$ fosse outro, a equação de realimentação poderia não ser linear, como no caso em que o modelo considera entradas exógenas, para o qual a realimentação é uma função afim. No entanto, para $\mathbb{H}_{0}$ o controle é linear e escreve-se

$$
u_{k}=G_{k} x_{k}
$$

em que as matrizes $G_{k} \in \mathbb{R}^{m \times n}, \forall k \in \mathscr{H} \backslash\{T\}$ são chamadas de ganhos do controle e não dependem de $\theta$.

Independentes e identicamente distribuídos.

Caso não seja a matriz identidade, mais adiante, a seu tempo, será indicada a alteração necessária.

Quando se tem informação completa do estado $\left(x_{k}, \theta_{k}\right)$, usa-se um controle linear dependente de $\theta$ da forma $u_{k}=G_{k}^{\theta_{k}} x_{k}$, encontrado nas Equações de Riccati Acopladas. 
Como pode-se notar até agora, o modelo é aleatório e pouco tratável. Por esse motivo, opta-se por reescrevê-lo de forma determinística. Esse modelo determinístico para cálculo do custo, que será tratado no Capítulo 3, é baseado no valor esperado da matriz de segundo momento de $x$ dado $\theta$, como em $\mathbf{E}\left(x_{k} x_{k}^{\prime} \mathbb{1}_{\left\{\theta_{k}=i\right\}}\right)$ (valor esperado de $x_{k} x_{k}^{\prime}$ dado que $\theta_{k}=i$ ). Essa matriz de segundo momento de $x_{k}$ dado $\theta_{k}=i$ será denotada por $X_{i}^{k}$. Ela possibilita o cálculo do valor esperado de $\left\|x_{k}\right\|^{2}$, bastando tomar o traço: $\mathbf{E}\left(\left\|x_{k}\right\|^{2}\right)=\sum_{i \in \mathscr{N}} \mathbf{E}\left(\left\|x_{k} \mathbb{1}_{\left\{\theta_{k}=i\right\}}\right\|^{2}\right)=\sum_{i \in \mathscr{N}} \operatorname{tr}\left(X_{i}^{k}\right)$.

\subsection{Exemplos numéricos}

Nesta seção constam quatro exemplos numéricos unidimensionais para ilustrar o comportamento dos SLSMs: um exemplo sem controle, nem ruído aditivo; outro exemplo sem controle, mas com ruído aditivo; outro exemplo com um controle estabilizante escolhido sem nenhum critério específico, apenas para exemplificar, sem adição de ruído; e por fim um exemplo com ruído aditivo e controle. Nestes exemplos não foi utilizado o MV para cálculo da solução. Portanto, parâmetros $C$ e $D$ do modelo $\mathbb{H}_{0}$ não serão apresentados.

Exemplo 3. (Sem controle e sem ruído) Considere $n=m=p=1$, ou seja, $x, u, \omega \in \mathbb{R}^{1}$, e a cadeia de Markov $\left\{\mathscr{N}, \mathbb{P}, \pi_{0}\right\}$, com $\mathscr{N}=\{1,2,3\}$, em que $\pi_{0}=(0.3,0.3,0.4)^{\prime}$. Os parâmetros $\Phi_{\theta}$ são $A=\{1.1,1,0.9\}, B=\{0,0,0\}$ e $H=\{0,0,0\}$. É dado $x_{0}=10$.

$$
\mathbb{P}=\left[\begin{array}{ccc}
0.96 & 0.02 & 0.02 \\
0.02 & 0.96 & 0.02 \\
0.02 & 0.02 & 0.96
\end{array}\right] \quad \begin{array}{cccc}
A_{1}=1.1 & \text { (modo ampliar) } & B_{1}=0 & H_{1}=0 \\
A_{2}=1 & \text { (modo conservar) } & B_{2}=0 & H_{2}=0 \\
A_{3}=0.9 & \text { (modo reduzir) } & B_{3}=0 & H_{3}=0
\end{array}
$$

Figura 10 - Uma realização da cM e a respectiva trajetória de $x_{k}$.
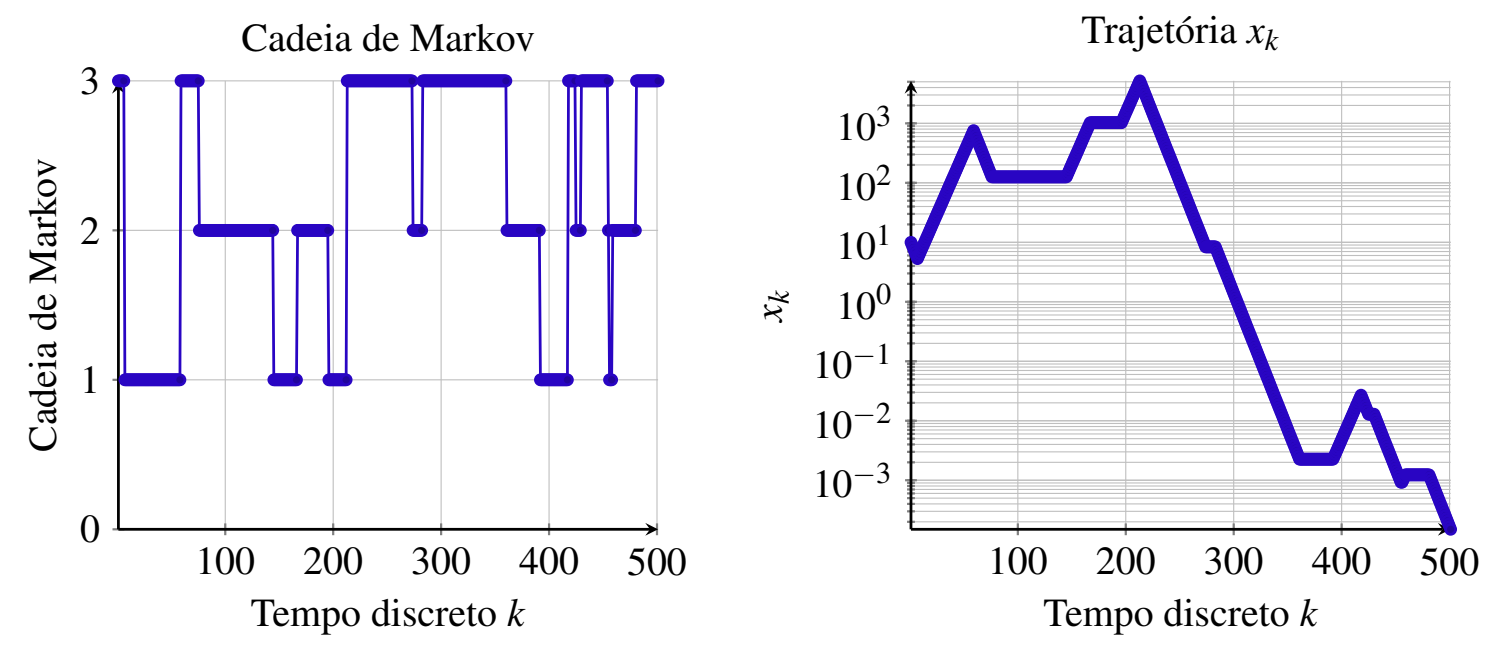

Perceba na Figura 10 o comportamento aleatório da cM e o respectivo efeito na trajetória do sistema dinâmico, o qual caminha "sem rumo" e se afasta bastante de zero.

Uma realização de $x_{k}$ consiste em um sorteio da $\mathrm{cM}$ e do ruído $\omega_{k}$ quando houver, além de fazer a evolução do SLSM pelo modelo $\mathbb{H}_{0}$. 
Figura 11 - A trajetória do SLSM para várias simulações da cM e o valor esperado $\mathbf{E}\left(\left\|x_{k}\right\|^{2} \mathbb{1}_{\left\{\theta_{k}\right\}}\right)$.

Várias realizações de $x_{k}$

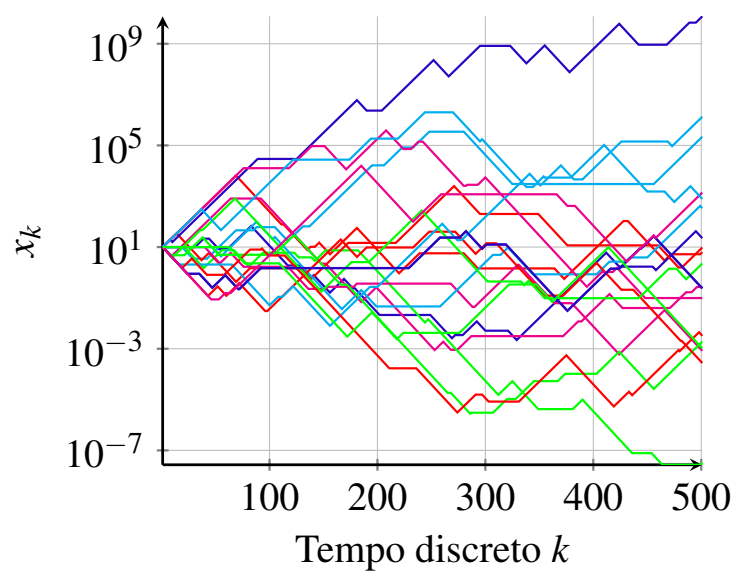

Trajetória de $X^{k}$

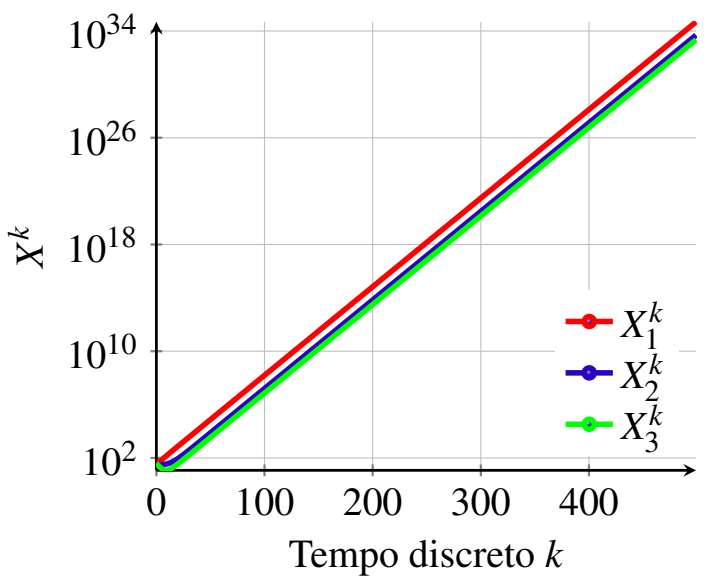

Figura 12 - Uma realização da cM e a respectiva trajetória de $x_{k}$.
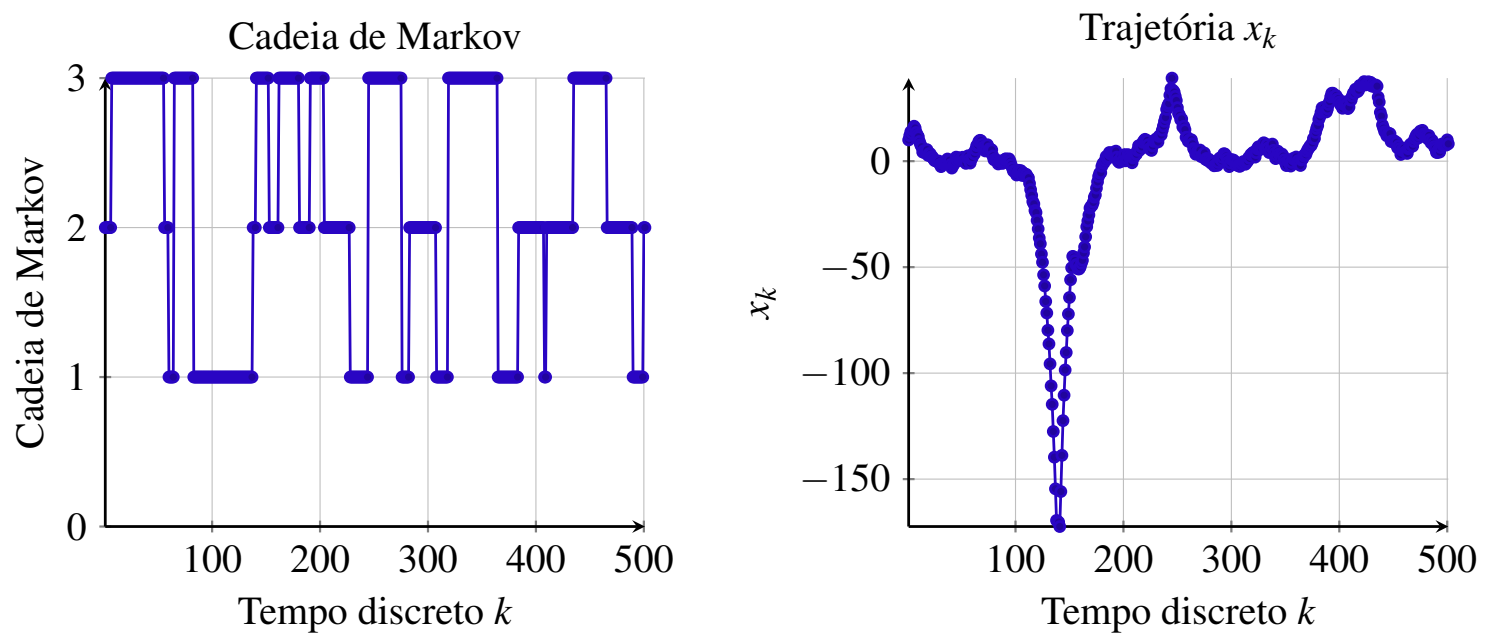

Já na Figura 11 é possível perceber mais nitidamente esse comportamento aleatório do SLSM com as diversas realizações do sistema dinâmico, fazendo parecer uma ramificação a partir do ponto de origem. Note ainda que $x_{k}$ às vezes pode ir para zero e às vezes pode tender ao infinito, pois o sistema não está sendo controlado. Por fim, veja o gráfico do valor esperado da norma quadrática de $x_{k}$ para cada um dos estados markovianos. Note que para todo $\theta, X_{\theta}^{k}$ tende ao infinito, pois a trajetória não está sendo corrigida (controlada).

Exemplo 4. (Sem controle e com ruído) Considere o mesmo problema do Exemplo 3. Mas agora com $H=\{0.1,0.3,0.5\}$ ( $\Phi$ é outro conjunto de parâmetros). É dado $x_{0}=10$. Além da aleatoriedade da cM, o SLSM representado na Figura 12 tem um ruído de média zero e variância 1. Com essa nova incerteza, o sistema que passava apenas por números positivos agora pode passar aos negativos, como se observa no gráfico. Note o afastamento do zero a partir de $k=100$, uma vez que o sistema não está sendo controlado.

O valor esperado da norma quadrática de $x_{k}$ também tende ao infinito neste exemplo, por não haver controle de sua trajetória, como se vê na Figura 13. 
Figura 13 - A trajetória do SLSM para várias simulações da cM e o valor esperado $\mathbf{E}\left(\left\|x_{k}\right\|^{2} \mathbb{1}_{\left\{\theta_{k}\right\}}\right)$.
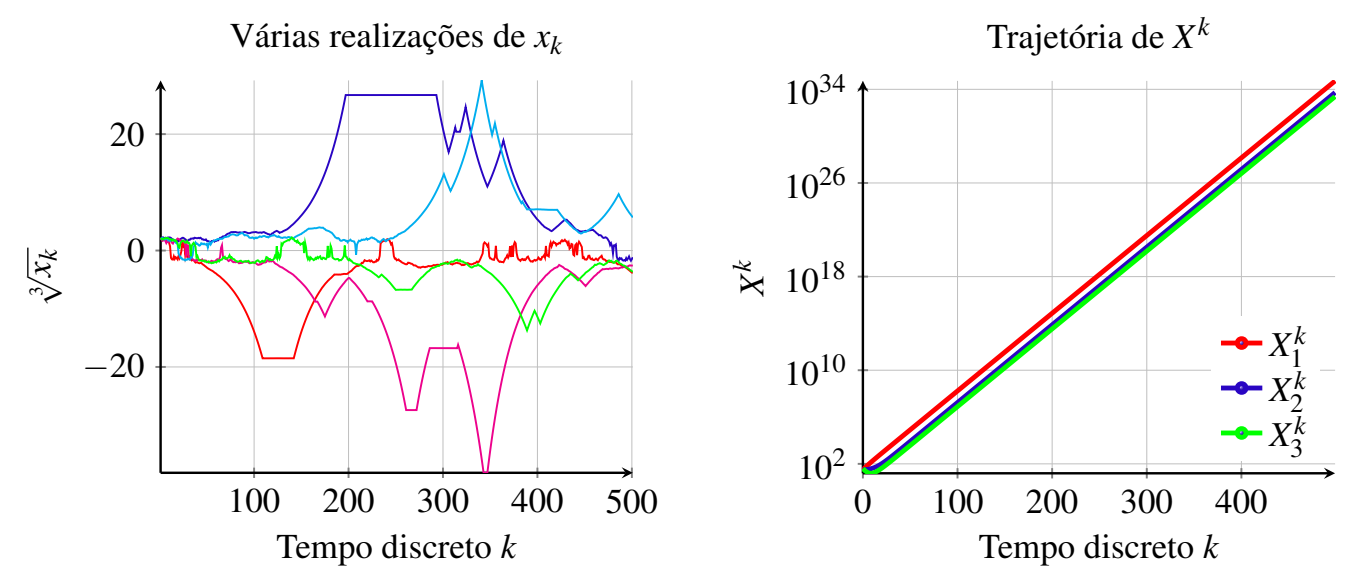

Exemplo 5. (Com controle e sem ruído) Considere ainda o mesmo problema do Exemplo 3, mas com os parâmetros $B=\{1,3,2\}$ e $H=\{0,0,0\}$. É dado $x_{0}=10$ e um ganho constante no tempo $G_{k}=-0.3 \forall k$ (não é o ganho ótimo):

Figura 14 - Uma realização da cM e a respectiva trajetória de $x_{k}$.
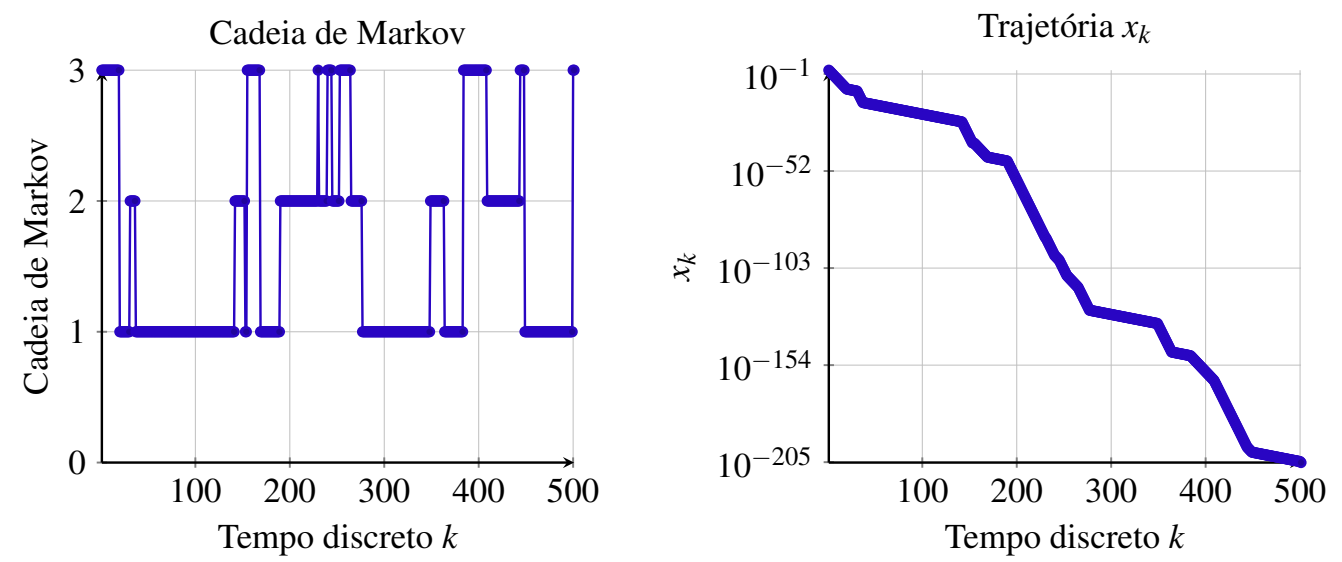

Figura 15 - A trajetória do SLSM para várias simulações da cM e o valor esperado $\mathbf{E}\left(\left\|x_{k}\right\|^{2} \mathbb{1}_{\left\{\theta_{k}\right\}}\right)$.
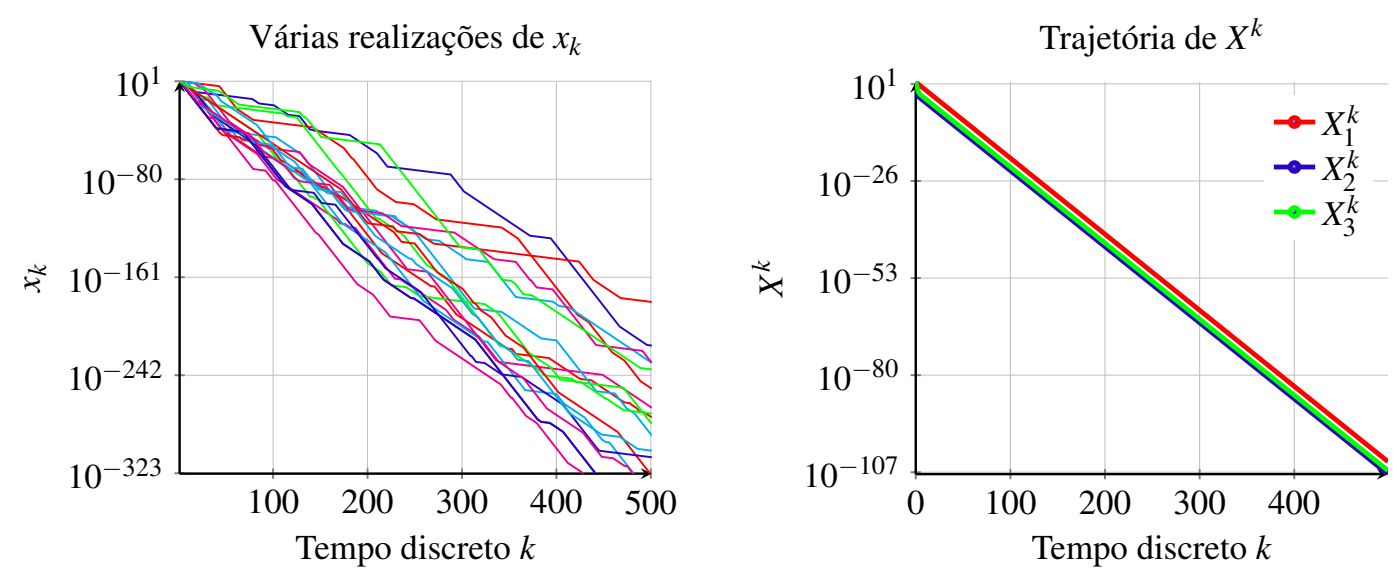
Neste exemplo, é notória a convergência do sistema para zero, uma vez que o ganho $G_{k}$ utilizado estabiliza o sistema. Como não há ruído, o controle aplicado faz a trajetória convergir para a origem, como se identifica na Figura 14. O fato é mais evidente olhando as diversas realizações da cM na Figura 15, bem como $X^{k}$.

Exemplo 6. (Com controle e ruído) Considere o problema do Exemplo 3. Os parâmetros $\Phi_{\theta}$ são $A=\{1.1,1,0.9\}, B=\{1,3,2\}$ e $H=\{0.1,0.3,0.5\}$. É dado $x_{0}=10$ e um ganho constante no tempo $G_{k}=-0.3 \forall k$ (não é o ganho ótimo):

Figura 16 - Uma realização da cM e a respectiva trajetória de $x_{k}$.
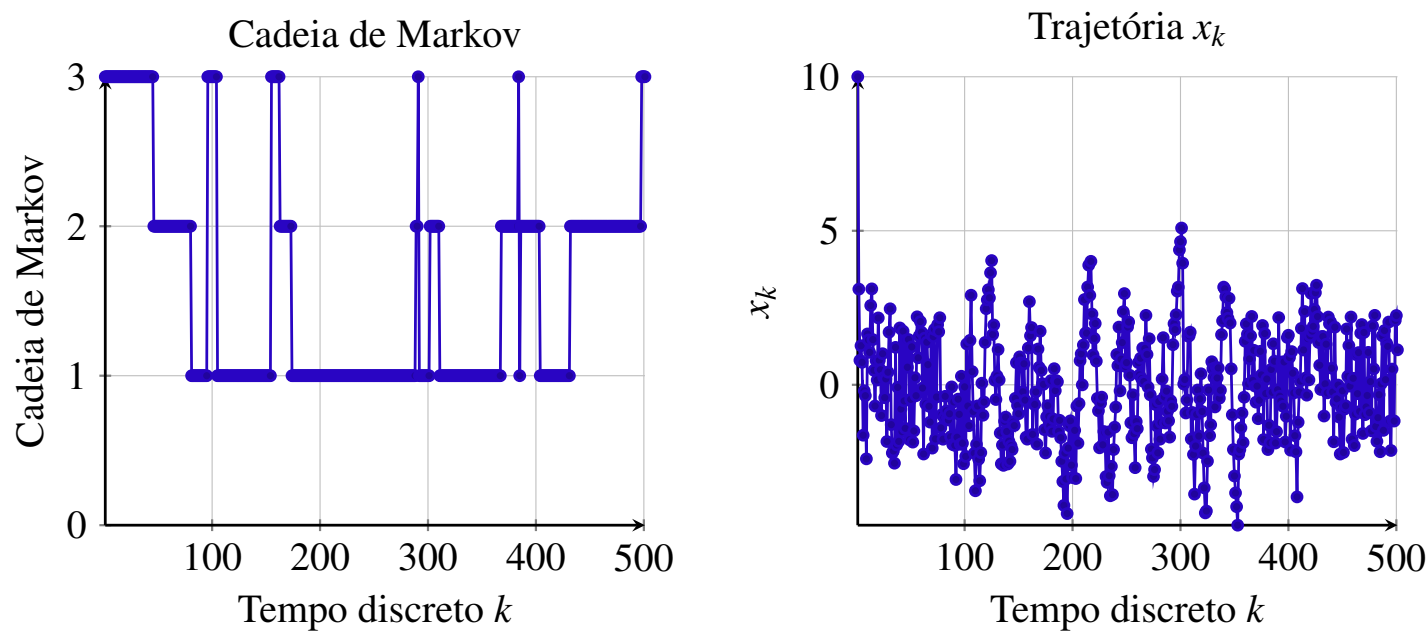

Figura 17 - A trajetória do SLSM para várias simulações da cM e o valor esperado $\mathbf{E}\left(\left\|x_{k}\right\|^{2} \mathbb{1}_{\left\{\theta_{k}\right\}}\right)$.
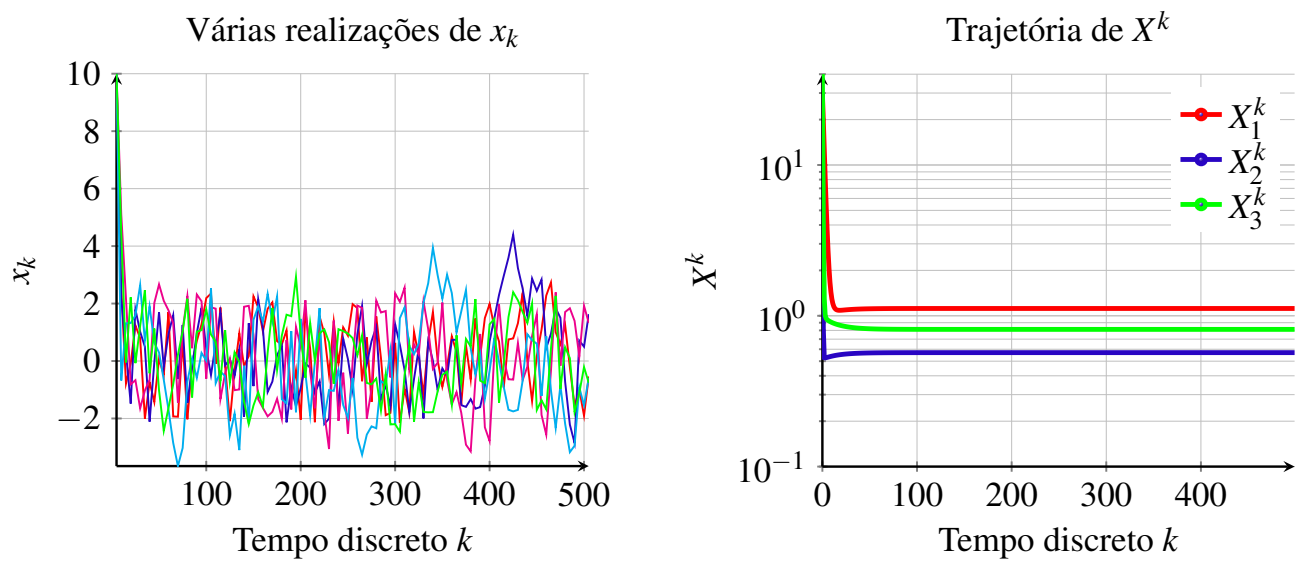

Neste último cenário, em que há ruído adicionado ao sistema, percebe-se que o ganho $G_{k}$ escolhido estabiliza-o. Veja na Figura 16 que o sistema controlado permanece orbitando a origem, pois o controle corrige a trajetória toda vez que o sistema tenta se afastar dela.

Na Figura 17 pode-se verificar várias trajetórias diferentes para o sistema controlado, todas com comportamento semelhante. Note que a norma quadrática de $x_{k}$ dessa vez não tende a zero, mas perceba que é limitada, diferentemente dos casos sem controle, em que tendia ao infinito. 
Veja que nos dois exemplos com controle, este não necessitou da informação de $\theta$, pois foi mantido constante ao longo do horizonte, não importando o valor da cM, de forma que $G_{k}=-0.3$ é um ganho estabilizante para o cenário sem observação dos saltos markovianos. O ganho que costuma-se chamar de ótimo é aquele que estabiliza o sistema com o mínimo de energia possível. Outros ganhos que estabilizam o sistema são considerados subótimos.

Observação 3 (Estabilidade). Perceba nos exemplos anteriores que quando a média $X_{\theta_{k}}^{k}=$ $\mathbf{E}\left(x_{k} x_{k}^{\prime} \mathbb{1}_{\left\{\theta_{k}\right\}}\right)$ permanece limitada ao longo do horizonte $k \in \mathscr{H}$, então o SLSM permanece limitado (estável), como é o caso do Exemplo 5 e do Exemplo 6, fato que é o objetivo final do controle RLQ. Quando isso não acontece, o sistema é instável, possibilitando trajetórias divergentes, como é o caso do Exemplo 3 e do Exemplo 4. 
CAPÍTULO

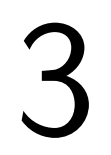

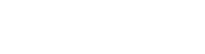

\section{MODELO DETERMINÍSTICO E MÉTODO VARIACIONAL}

Neste capítulo é tratado o modelo determinístico baseado no valor esperado da matriz de segundo momento de $x$, bem como aponta uma condição necessária de otimalidade já bastante explorados na literatura, de modo que serão apresentados em formulários. O método variacional é descrito e explicado, bem como apresentado um algoritmo ilustrando-o.

\subsection{O custo determinístico}

É interessante notar que, ao se considerar a Equação 2.5 no modelo RLQ da Definição 2, pode-se trocar a variável $u_{k}$ por $G_{k}$. Assim, dado um ganho $G_{k}$ e definindo as coleções auxiliares

$$
\overline{\mathbf{A}}^{k}=A+B \bullet G_{k} \quad \text { e } \quad \overline{\mathbf{C}}^{k}=C+G_{k}^{\prime} \bullet D \bullet G_{k},
$$

(chamadas matrizes de malha fechada ou em inglês closed loop), o sistema $\mathbb{H}_{0}$ da Equação 2.4a $-2.4 \mathrm{~b}$ passa a ser escrito como

$$
\mathbb{H}_{1}\left\{\begin{array}{l}
x_{k+1}=\overline{\mathbf{A}}_{\theta_{k}}^{k} x_{k}+H_{\theta_{k}} \omega_{k} \\
z_{k}=x_{k}^{\prime} \overline{\mathbf{C}}_{\theta_{k}}^{k} x_{k}
\end{array}\right.
$$

em que a ação de controle é dada por $G_{k}$ e já está “embutida” em $\overline{\mathbf{A}}$ e $\overline{\mathbf{C}}$.

Definição 1. Definem-se alguns operadores que serão utilizados no modelo mais adiante. Considere as coleções de matrizes $U \in \mathbb{V}_{n \times n}^{\mathscr{N}}$ e $V \in \mathbb{V}_{n \times n}^{\mathscr{N}}$ arbitrárias. Assim, definem-se

$$
\left\{\begin{array}{l}
\mathscr{T}_{V}^{i}(U)=\sum_{j \in \mathscr{N}} \mathbb{P}_{j i} V_{j} U_{j} V_{j}^{\prime}, \\
\mathscr{E}^{i}(U)=\sum_{j \in \mathscr{N}} \mathbb{P}_{i j} U_{j}, \\
\mathscr{L}_{V}^{i}(U)=V_{i}^{\prime} \mathscr{E}^{i}(U) V_{i} .
\end{array}\right.
$$


O resultado a seguir será deixado como um formulário, pois na literatura já tem várias menções do mesmo com notação diversa.

Definição 2 (Política de controle e variável $X$ ). Dada uma sequência de ganhos $\left\{\left.G\right|_{0} ^{T-1}\right\}=$ $\left\{G_{0}, G_{1}, \ldots, G_{T-1}\right\}$ chamada de política de controle, para se obter o valor esperado $\mathbf{E}\left(x_{k} x_{k}^{\prime} \mathbb{1}_{\left\{\theta_{k}=i\right\}}\right)=X_{i}^{k}$ ao longo de $k \in \mathscr{H}$, usando-se a Equação 3.3a, tem-se para cada $i \in \mathscr{N}$ :

$$
\left\{\begin{array}{l}
X_{i}^{0}=x_{0} x_{0}^{\prime} \pi_{0}^{i}, \\
X_{i}^{k+1}=\mathscr{T}_{\overline{\mathbf{A}}^{k}}^{i}\left(X^{k}\right)+\Sigma_{i}^{k}, \\
\Sigma_{i}^{k}=\sum_{j \in \mathscr{N}} \mathbb{P}_{j i} \pi_{k}^{j} H_{j} H_{j}^{\prime},
\end{array}\right.
$$

de forma que as matrizes $\Sigma_{i}^{k}$ representam a covariância do ruído aditivo $\omega_{k}$ ponderada pela distribuição de $\theta_{k}$. Note a matriz de malha fechada $\overline{\mathbf{A}}$ na Equação $3.4 \mathrm{~b}$.

Observação 4. Algumas propriedades imediatas dessa definição são que $X_{i}^{k}$ e $\Sigma_{i}^{k}$ são matrizes simétricas e positivas semidefinidas para todo $i \in \mathscr{N}$ e para todo $k \in \mathscr{H}$ (ou seja, $X=X^{\prime} \geq 0 \mathrm{e}$ $\Sigma=\Sigma^{\prime} \geq 0$ ). Além disso, $X^{0}$ é uma coleção de matrizes de posto 1 , e portanto singular.

Definição 3 (Custo total determinístico). Dada uma política de controle $\left\{\left.G\right|_{0} ^{T-1}\right\}$ arbitrária, considerando a Equação 3.1 e a Equação 2.3, calcula-se o custo total $\mathbf{J}$ do horizonte de planejamento $\mathscr{H}$ dessa política de forma determinística pela seguinte equação:

$$
\mathbf{J}\left(\left\{\left.G\right|_{0} ^{T-1}\right\}\right)=\sum_{k \in \mathscr{H} \backslash\{T\}} J_{k}=\sum_{k \in \mathscr{H} \backslash\{T\}}\left\langle X^{k}, \overline{\mathbf{C}}^{k}\right\rangle .
$$

em que o valor esperado de $z_{k}$ da Equação $3.2 b$ é dado por

$$
J_{k}=\mathbf{E}\left(z_{k}\right)=\mathbf{E}\left(x_{k}^{\prime} \overline{\mathbf{C}}_{\theta_{k}}^{k} x_{k}\right)=\sum_{i \in \mathscr{N}} \operatorname{tr}\left(x_{k} x_{k}^{\prime} \overline{\mathbf{C}}_{i}^{k} \mathbb{1}_{\left\{\theta_{k}=i\right\}}\right)=\left\langle X^{k}, \overline{\mathbf{C}}^{k}\right\rangle
$$

Para resolver o problema de controle RLQ, cuja dimensão é de $m \times n \times T$, quebra-se-lhe em subproblemas de dimensão $m \times n$. Para tanto, introduzem-se mais duas variáveis $L$ e $\sigma$ para calcular o custo total $\mathbf{J}$.

Definição 3 (Custo total). Dada uma política de controle $\left\{\left.G\right|_{\ell} ^{T-1}\right\}$, considerando a soma dos custos de $J_{\ell}$ a $J_{T-1}$ (chamada custo de continuação a partir de $k=\ell$ ) e as equações (3.1) e (2.2), tem-se

$$
\left.J\right|_{\ell} ^{T-1}=\sum_{k=\ell}^{T-1} J_{k}=\left\langle X^{\ell}, L^{\ell}\right\rangle+\pi_{\ell}^{\prime} \sigma^{\ell},
$$

Veja por exemplo Bortolin (2012, Proposição 2.1) ou Oliveira (2014, Proposição 2.1).

Caso a covariância do ruído seja $W$ em vez da matriz identidade (veja a Definição 2), modifica-se o modelo acima para $\Sigma_{i}^{k}=\sum_{j \in \mathscr{N}} \mathbb{P}_{j i} \pi_{k}^{j} H_{j} W H_{j}^{\prime}$. 
demonstrado na Equação A.5, considerando-se o Definição 1, em que $L^{k} \in \mathbb{V}_{n \times n}^{\mathcal{N}}$ e $\sigma^{k} \in \mathbb{V}_{1 \times 1}^{\mathcal{N}}$ para todo $k \in \mathscr{H}$, e:

$$
\left\{\begin{array}{l}
L_{i}^{T}=0_{n \times n}, \sigma_{i}^{T}=0, \\
L_{i}^{k}=\overline{\mathbf{C}}_{i}^{k}+\mathscr{L}_{\overline{\mathbf{A}}^{k}}^{i}\left(L^{k+1}\right), \\
\sigma_{i}^{k}=\operatorname{tr}\left(\mathscr{E}^{i}\left(L^{k+1}\right) H_{i} H_{i}^{\prime}\right)+\mathscr{E}^{i}\left(\sigma^{k+1}\right) .
\end{array}\right.
$$

Nota-se então, que o custo da política $\left\{\left.G\right|_{0} ^{T-1}\right\}$ definido pelo somatório em (3.5) é dado pelo produto interno das coleções $X, L, \pi$ e $\sigma$, conforme

$$
\mathbf{J}\left(\left\{\left.G\right|_{0} ^{T-1}\right\}\right)=\left\langle X^{0}, L^{0}\right\rangle+\pi_{0}^{\prime} \sigma^{0} .
$$

Observação 5. Uma propriedade imediata dessa definição é que $L_{i}^{k}$ é matriz simétrica positiva semidefinida para todo $i \in \mathscr{N}$ e para todo $k \in \mathscr{H}$ (ou seja, $L=L^{\prime} \geq 0$ ). Diferentemente de $X$ que evolui no tempo usando o operador $\mathscr{T}_{(\cdot)}(\cdot)$, note a regressão no tempo para $L$ usando o operador $\mathscr{L}_{(\cdot)}(\cdot)$ e $\sigma$, ou seja, inicia-se $\operatorname{com} k=T$ e volta-se no tempo em direção a $k=0$.

\subsection{Método variacional}

Definidos todos os elementos necessários para entender o problema e a formulação determinística para o cálculo do custo, apresentam-se os principais resultados deste capítulo e o algoritmo variacional.

Definição 4 (Problema variacional). O problema variacional consiste em minimizar o custo de continuação dado pela Equação 3.6, para cada $k=T-1, T-2, \ldots, 1,0$ :

$$
\left\{\begin{array}{l}
\left.\min _{G_{k} \in \mathbb{R}^{m \times n}} J\right|_{k} ^{T-1} \\
\text { sujeito às equações da Definição } 2 \text { e da Definição } 3 .
\end{array}\right.
$$

Uma condição necessária de otimalidade da forma "derivadas parciais iguais a zero" aplicada ao custo de continuação permite escrever o próximo resultado.

Proposição 1 (Otimalidade). Seja $\Lambda_{i}^{k+1}=\mathscr{E}^{i}\left(L^{k+1}\right)$. O ganho $G_{k}$ que resolve o problema variacional dado na Equação 3.9 é solução do sistema linear:

$$
\sum_{i \in \mathscr{N}}\left[\left(D_{i}+B_{i}^{\prime} \Lambda_{i}^{k+1} B_{i}\right) G_{k}+B_{i}^{\prime} \Lambda_{i}^{k+1} A_{i}\right] X_{i}^{k}=0 .
$$

A demonstração pode ser vista na Equação A.8.

Para resolver o sistema linear acima, usa-se sua forma vetorizada:

$$
\sum_{i \in \mathscr{N}}\left[X_{i}^{k} \otimes\left(D_{i}+B_{i}^{\prime} \Lambda_{i}^{k+1} B_{i}\right)\right] \operatorname{vec}\left(G_{k}\right)=-\sum_{i \in \mathscr{N}} \operatorname{vec}\left(B_{i}^{\prime} \Lambda_{i}^{k+1} A_{i} X_{i}^{k}\right),
$$

Esse é o sistema linear $\mathbb{S}_{k}^{\eta}$ referido na introdução. 
em que o operador vec $(\cdot)$ empilha as colunas de uma matriz, formando um vetor, e $\otimes$ representa o produto de Kronecker. Veja o exemplo de $\otimes$ e vec $(\cdot)$ abaixo:

$$
\begin{aligned}
& A=\left[\begin{array}{ll}
a_{11} & a_{12} \\
a_{21} & a_{22}
\end{array}\right], \quad \text { e } \quad B=\left[\begin{array}{ll}
b_{11} & b_{12}
\end{array}\right],
\end{aligned}
$$

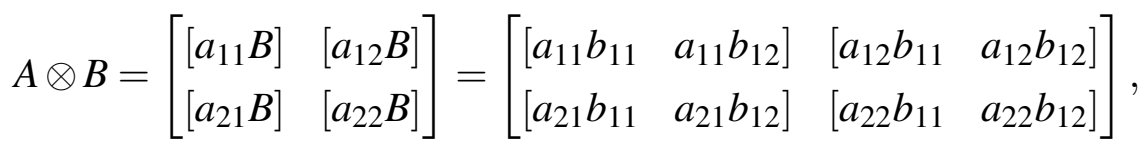

$$
\begin{aligned}
& \left.\operatorname{vec}(A)=\operatorname{vec}\left(\left[\left[\begin{array}{l}
a_{11} \\
a_{21}
\end{array}\right] \quad\left[\begin{array}{l}
a_{12} \\
a_{22}
\end{array}\right]\right]\right)=\left[\begin{array}{l}
a_{11} \\
a_{21} \\
a_{12} \\
a_{22}
\end{array}\right], \quad \operatorname{vec}(B)=\operatorname{vec}\left(\left[\begin{array}{ll}
\left.b_{11}\right] & {\left[b_{12}\right.}
\end{array}\right]\right]\right)=\left[\begin{array}{l}
b_{11} \\
b_{12}
\end{array}\right] .
\end{aligned}
$$

Definição 5 (Vetorização e sua inversa). Defina vec $(\cdot): \mathbb{R}^{m \times n} \rightarrow \mathbb{R}^{m n \times 1}, \operatorname{vec}(X)=x$ o operador que empilha as colunas $X_{r}$ da matriz $X, r=1, \ldots, n$, formando um vetor $x \in \mathbb{R}^{m n \times 1}$. Defina $\mathbf{v e c}_{m, n}^{-1}(\cdot): \mathbb{R}^{m n \times 1} \rightarrow \mathbb{R}^{m \times n}, \mathbf{v e c}_{m, n}^{-1}(x)=X$, o operador inverso de vec $(X)$, que recupera a matriz $X \in \mathbb{R}^{m \times n}$ a partir do vetor $x \in \mathbb{R}^{m n \times 1}$ (reshape).

Observação 6. O cálculo dos ganhos $G_{k}$ não precisa de $\sigma^{k}$, pois $\partial \sigma^{k} / \partial G_{k}=0$. Como a variável $X$ precisará ser atualizada de $k=0$ até $k=T$ a cada iteração $\eta$ do MV, é mais fácil calcular o custo total $\mathbf{J}$ somando as parcelas $\left\langle X^{k}, \overline{\mathbf{C}}^{k}\right\rangle$ conforme a Equação 3.5, em vez de usar a Equação 3.8.

Observação 7. Das Observações 4 e 5 , note que a matriz $X_{i}^{k} \otimes\left(D_{i}+B_{i}^{\prime} \Lambda_{i}^{k+1} B_{i}\right)$ do sistema é simétrica, pois os fatores o são, e positiva semidefinida para todo $i \in \mathscr{N}$, pois, de acordo com Zhou, Doyle e Glover (1996, p. 25), os autovalores de $P \otimes Q$ são os respectivos produtos dos autovalores de $P$ pelos autovalores de $Q$, que, no caso, são todos não negativos.

Observação 8. Os algoritmos Algoritmo 1, Algoritmo 2 e Algoritmo 3 a seguir consideram indexação a partir de 1 e não a partir de zero. Elementos de dimensões iguais estão alocados multiplicando por zero um deles que já está na memória.

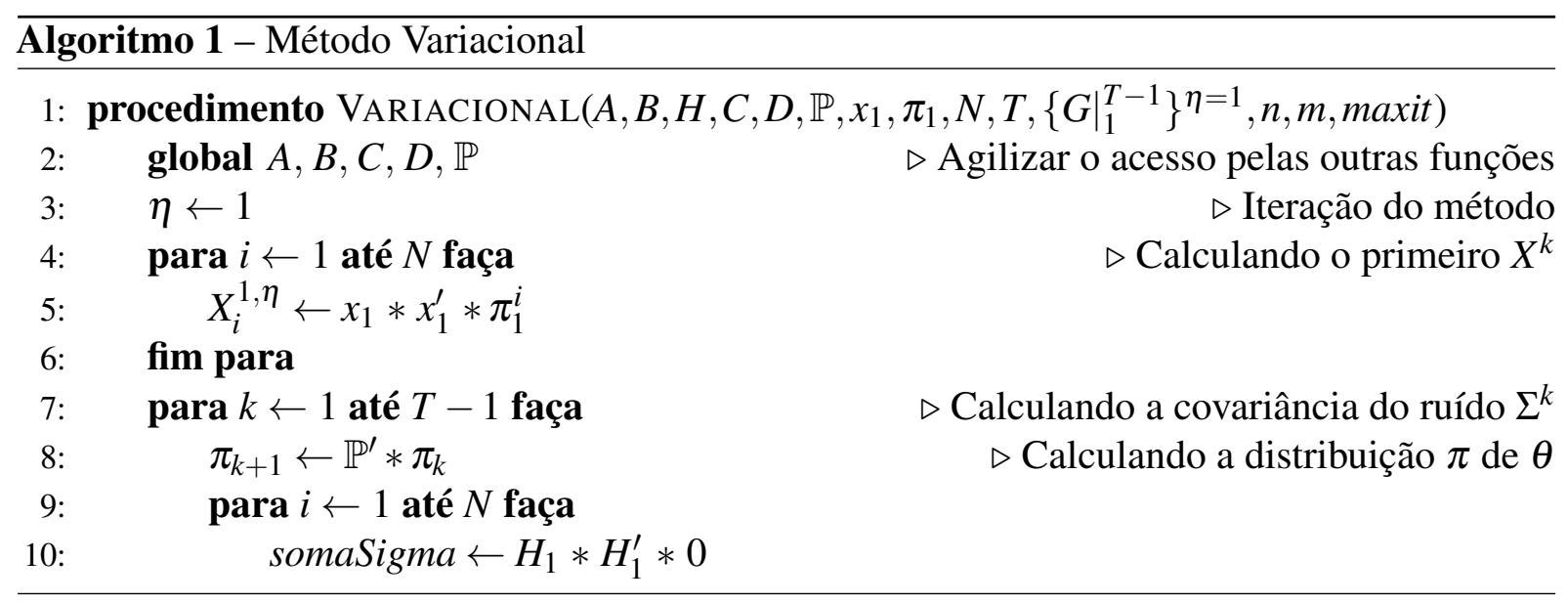




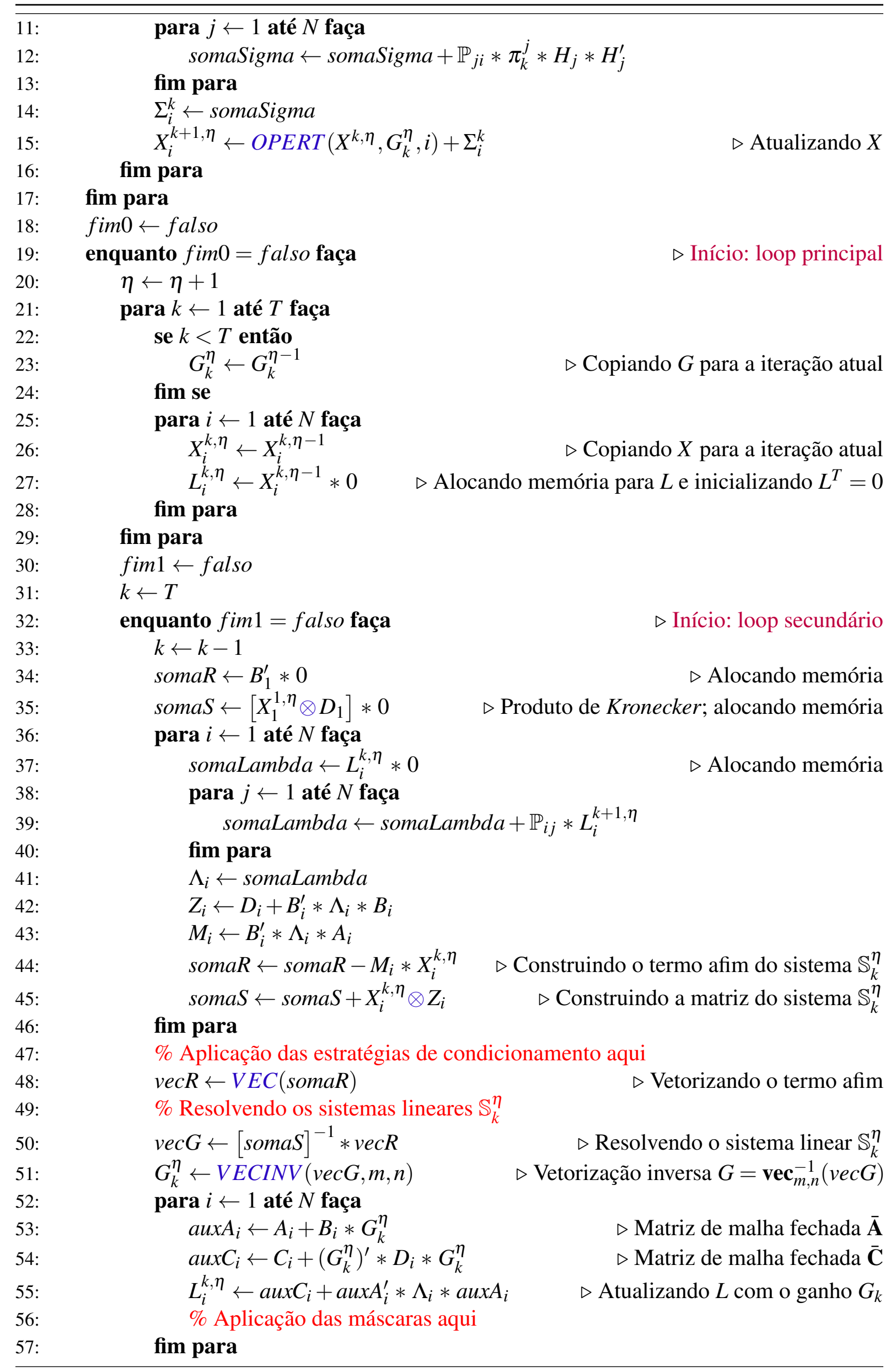



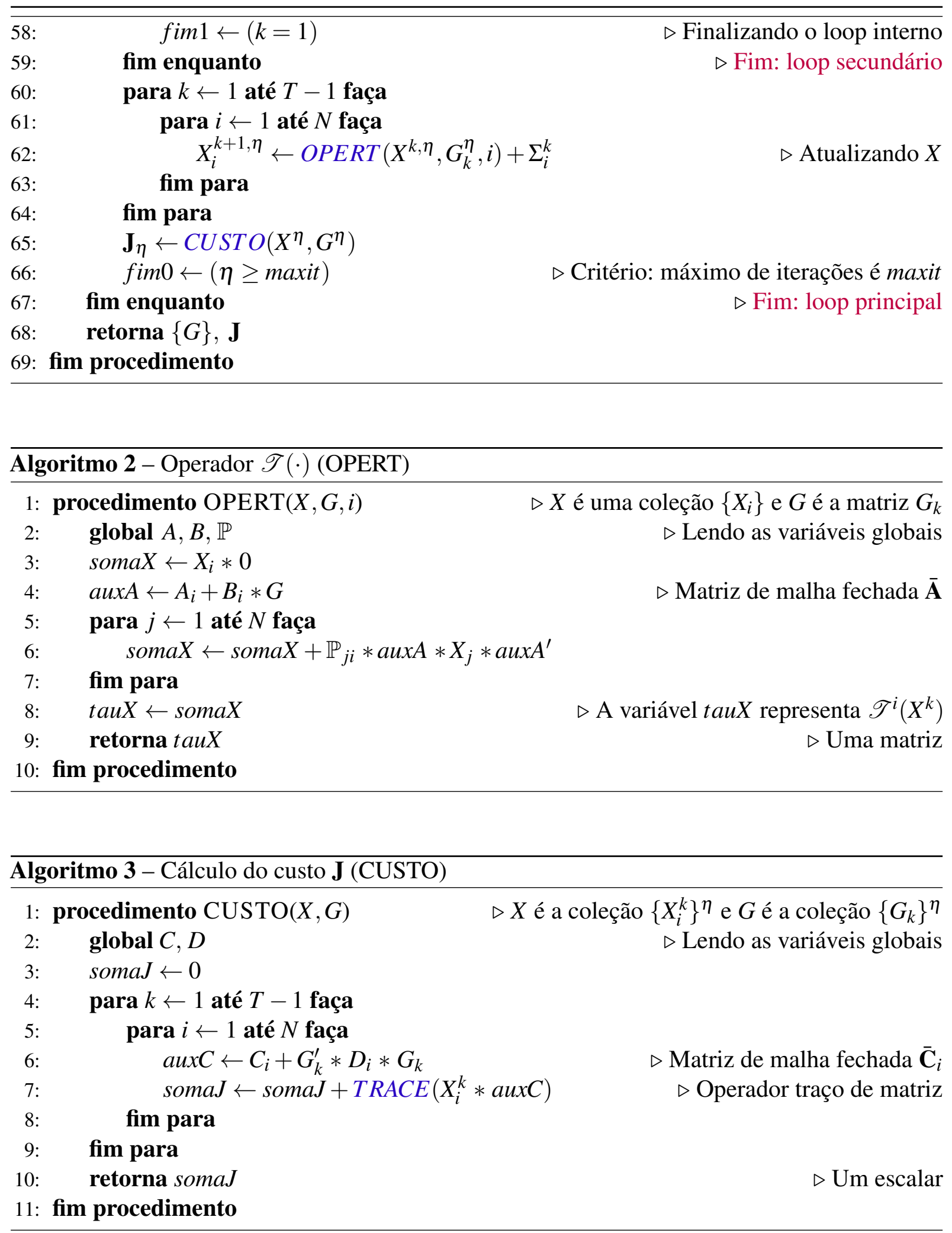

O Método Variacional funciona conforme o seguinte esquema, com $k \in \mathscr{H}=\{0,1,2,3,4\}$, (horizonte de tamanho $T=4$ ). Dada uma política de controle $\left\{G_{0}^{T-1}\right\}=\left\{G_{0}, G_{1}, G_{2}, G_{3}\right\}$,

$$
\begin{aligned}
& X^{0} \stackrel{G_{0}, \Sigma^{0}}{\longrightarrow} X^{1} \stackrel{G_{1}, \Sigma^{1}}{\longrightarrow} X^{2} \stackrel{G_{2}, \Sigma^{2}}{\longrightarrow} X^{3} \stackrel{G_{3}, \Sigma^{3}}{\longrightarrow} \quad X^{4} \\
& L^{0} \stackrel{G_{0}}{\longleftarrow} L^{1} \stackrel{G_{1}}{\longleftarrow} L^{2} \quad \stackrel{G_{2}}{\longleftarrow} L^{3} \stackrel{G_{3}}{\longleftarrow} L^{4}=0 .
\end{aligned}
$$


O MV fixa todos os ganhos $G_{r}$ e atualiza apenas um deles $G_{k}$, resolvendo-se um sistema linear $\mathbb{S}_{k}$, e este ganho atualizado, por sua vez, é fixado na próxima etapa da iteração. O sistema linear $\mathbb{S}_{k}^{\eta}$, conforme descrito na Equação 3.11, está indicado:

$$
\begin{aligned}
& \left\{G_{0}^{\eta-1}, G_{1}^{\eta-1}, G_{2}^{\eta-1}, G_{3}^{\eta-1}\right\}, \text { fim da iteração } \eta-1 \\
& \left\{G_{0}^{\eta-1}, G_{1}^{\eta-1}, G_{2}^{\eta-1}, G_{3}^{\eta}\right\} \quad \mathbb{S}_{k}^{\eta}, k=3 \\
& \left\{G_{0}^{\eta-1}, G_{1}^{\eta-1}, \quad G_{2}^{\eta}, \quad G_{3}^{\eta}\right\} \quad \mathbb{S}_{k}^{\eta}, k=2 \\
& \left\{G_{0}^{\eta-1}, \quad G_{1}^{\eta}, \quad G_{2}^{\eta}, \quad G_{3}^{\eta}\right\} \quad \mathbb{S}_{k}^{\eta}, k=1 \\
& \left\{G_{0}^{\eta}, \quad G_{1}^{\eta}, \quad G_{2}^{\eta}, \quad G_{3}^{\eta}\right\} \quad \mathbb{S}_{k}^{\eta}, k=0 \\
& \left\{G_{0}^{\eta}, G_{1}^{\eta}, \quad G_{2}^{\eta}, G_{3}^{\eta}\right\}, \text { fim da iteração } \eta \\
& \left\{G_{0}^{\eta}, \quad G_{1}^{\eta}, \quad G_{2}^{\eta}, \quad G_{3}^{\eta+1}\right\} \quad \mathbb{S}_{k}^{\eta+1}, k=3 \\
& \left\{G_{0}^{\eta}, G_{1}^{\eta}, G_{2}^{\eta+1}, G_{3}^{\eta+1}\right\} \quad \mathbb{S}_{k}^{\eta+1}, k=2 \\
& \left\{G_{0}^{\eta}, G_{1}^{\eta+1}, G_{2}^{\eta+1}, G_{3}^{\eta+1}\right\} \quad \mathbb{S}_{k}^{\eta+1}, k=1 \\
& \left\{G_{0}^{\eta+1}, G_{1}^{\eta+1}, G_{2}^{\eta+1}, G_{3}^{\eta+1}\right\} \quad \mathbb{S}_{k}^{\eta+1}, k=0 \\
& \left\{G_{0}^{\eta+1}, G_{1}^{\eta+1}, G_{2}^{\eta+1}, G_{3}^{\eta+1}\right\}, \text { fim da iteração } \eta+1
\end{aligned}
$$



CAPÍTULO

4

IMPLEMENTAÇÕES

Como há muitos sistemas lineares $\mathbb{S}_{k}$ mal condicionados ao longo das iterações do MV, serão feitas mudanças de base para melhorar o condicionamento dos mesmos.

Quando o condicionamento é ruim, pequenas perturbações em $A$ ou em $b$ do sistema $A x=b$ provocam grandes perturbações na solução $x$, e a imagem de $A$ é uma deformação muito acentuada do espaço, como ilustrado na Figura 18.

Definição 6 (Condicionamento). O condicionamento numérico de uma matriz $A$ é definido como o produto das normas de $A$ e sua inversa, e, segundo Nocedal e Wright (2006, p. 43), pode ainda ser descrito como a razão cond $(A)$ entre o maior e o menor autovalor $\mu$ :

$$
\operatorname{cond}(A)=\|A\| \cdot\left\|A^{-1}\right\|=\frac{\left|\mu_{\max }(A)\right|}{\left|\mu_{\min }(A)\right|} .
$$

Segundo explicado por Franco (2013, Seção 4.8), se $A$ é conhecida exatamente e se faz uma perturbação no parâmetro $b$ da forma $b+\delta b$, a solução $x$ será perturbada para $x+\delta x$ de modo que, no novo sistema linear

$$
A(x+\delta x)=b+\delta b, \quad \operatorname{com} \quad \frac{\|\delta x\|}{\|x\|} \leq \operatorname{cond}(A) \frac{\|\delta b\|}{\|b\|} .
$$

Também, se $b$ é conhecido exatamente e $A$ for perturbada de $\delta A$, então

$$
(A+\delta A)(x+\delta x)=b, \quad \text { e } \quad \frac{\|\delta x\|}{\|x+\delta x\|} \leq \operatorname{cond}(A) \frac{\|\delta A\|}{\|A\|} .
$$

E por fim, se $A$ e $b$ forem perturbados, segundo Cunha (2000, p. 43),

$$
(A+\delta A)(x+\delta x)=b+\delta b, \quad \text { e } \quad \frac{\|\delta x\|}{\|x\|} \leq \frac{\operatorname{cond}(A)}{1-\operatorname{cond}(A) \frac{\|\delta A\|}{\|A\|}}\left(\frac{\|\delta A\|}{\|A\|}+\frac{\|\delta b\|}{\|b\|}\right) .
$$

Nos três casos se percebe que, se o número de condição de $A$ é grande, o limitante do erro relativo da solução $x$ também é grande. Cunha (2000, p. 43) explica que, dada uma calculadora 
que usa $p$ dígitos, se cond $(A)$ é da ordem de $10^{q}$, então, baseando-se nos limitantes dos erros, a solução pode ter apenas $p-q$ dígitos significativos confiáveis.

Figura 18 - A transformação linear deforma o espaço quase em uma reta (exemplo em $\mathbb{R}^{2}$ ).

Algumas bolas $B_{r}$

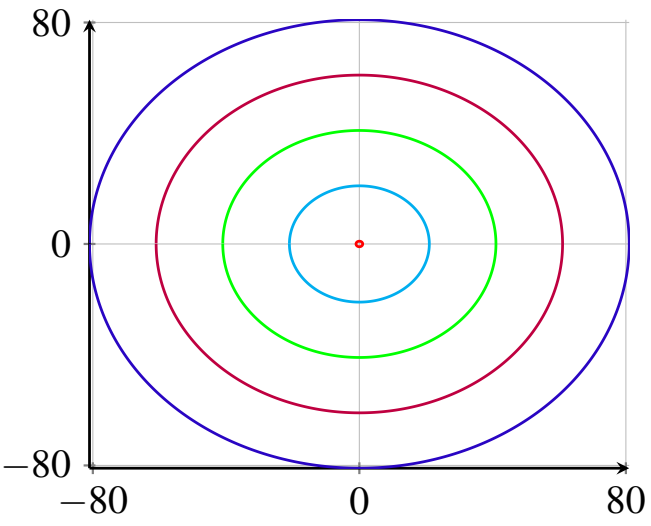

Imagens das bolas $B_{r}$ por $S$

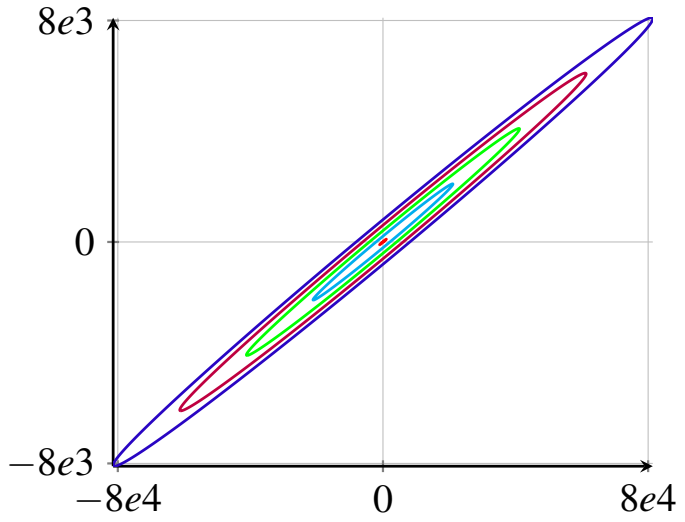

Uma das técnicas clássicas da literatura que tratam dessa deformação do espaço é a decomposição espectral (autovalores e autovetores). Nesse sentido, inspirando-se na formulação do método de Newton Modificado em Nocedal e Wright (2006, p. 52), objetiva-se fazer uma mudança de base na qual os autovalores estejam razoavelmente próximos entre si. Com isso, $\left|\mu_{\max }\right|$ estará próximo de $\left|\mu_{\min }\right|$ e cond $(A)$ será pequeno, possibilitando uma solução mais confiável.

No entanto, a decomposição espectral pode se tornar inviável quando a matriz não é perfeitamente simétrica, por ter autovalores complexos, ou singular, podendo ter pequenos pseudo autovalores negativos. Isso acabaria por contaminar os cálculos introduzindo componentes complexas desnecessárias. Por isso, foi escolhida uma decomposição que tem propriedades semelhantes no tocante ao condicionamento, a chamada Decomposição em Valores Singulares, mais conhecida pela sigla inglesa SVD (Singular Value Decomposition).

Em matrizes simétricas, que é o caso dos sistemas $\mathbb{S}_{k}$ do $\mathrm{MV}$, os valores singulares obtidos na SVD são exatamente o valor absoluto dos autovalores da matriz, conforme afirmado por Trefethen e Bau III (1997, Theorem 5.5).

A Decomposição em Valores Singulares para uma matriz A quadrada é tal que

$$
A=U \Sigma V^{\prime}
$$

em que $U$ e $V$ são matrizes ortonormais, ou seja, $U U^{\prime}=U^{\prime} U=I=V V^{\prime}=V^{\prime} V$ e $\Sigma$ é uma matriz diagonal contendo os valores singulares $\sigma_{n} \geq \ldots \geq \sigma_{1} \geq 0$. Se, além disso, a matriz $A$ for simétrica, então os autovalores $\mu_{i}$ são reais e existe uma decomposição de autovalores em uma base $Q$ ortonormal $A=Q \Lambda Q^{\prime} \operatorname{com}|\Lambda|=P \Sigma P^{\prime}$ (para alguma matriz de permutação $P$ ), segundo Trefethen e Bau III (1997, Theorem 24.7). 
Com essas hipóteses, definindo a matriz $\operatorname{sign}(\Lambda)=[\operatorname{sign}(\Lambda)]_{i j}=\operatorname{sign}\left(\Lambda_{i j}\right)$, tem-se que

$$
A=Q \Lambda Q^{\prime}=Q|\Lambda| \operatorname{sign}(\Lambda) Q^{\prime}=Q P \Sigma P^{\prime} \operatorname{sign}(\Lambda) Q^{\prime}=(Q P) \Sigma(Q \operatorname{sign}(\Lambda) P)^{\prime} \stackrel{(4.1)}{=} U \Sigma V^{\prime},
$$

Por comparação, e pela unicidade da SVD, tem-se $U=Q P$ e $V=Q \operatorname{sign}(\Lambda) P$, já que $P, Q, U$ e $V$ são matrizes ortonormais, além do que

$$
V^{\prime} U=P^{\prime} \operatorname{sign}(\Lambda) Q^{\prime} Q P=P^{\prime} \operatorname{sign}(\Lambda) P
$$

A mudança de base para o problema variacional descrita na Equação 4.7 é tal que

$$
\mathbf{V}^{\prime} A \mathbf{V} \vec{a}=\mathbf{V}^{\prime} b, \operatorname{com} x=\mathbf{V} \vec{a}, \mathbf{V} \in \mathbb{R}^{n \times n}, \vec{a} \in \mathbb{R}^{n}
$$

Sendo a matriz $A$ do sistema $\mathbb{S}_{k}$ simétrica, portanto $A=U \Sigma V^{\prime}$, escolhendo $\mathbf{V}=U[\Sigma]^{-\frac{1}{\alpha}}$ como base, em que $[\Sigma]^{-\frac{1}{\alpha}}$ representa a inversa da raiz $\alpha$-ésima da matriz $[\Sigma]$ :

$$
\begin{aligned}
\mathbf{V}^{\prime} A \mathbf{V} & =[\Sigma]^{-\frac{1}{\alpha}} U^{\prime}\left(U \Sigma V^{\prime}\right) U[\Sigma]^{-\frac{1}{\alpha}} \\
& =[\Sigma]^{-\frac{1}{\alpha}}\left(U^{\prime} U\right) \Sigma\left(P^{\prime} \operatorname{sign}(\Lambda) P\right)[\Sigma]^{-\frac{1}{\alpha}} \\
& =[\Sigma]^{-\frac{1}{\alpha}} \Sigma\left(P^{\prime} \operatorname{sign}(\Lambda) P\right)[\Sigma]^{-\frac{1}{\alpha}} \\
& =P^{\prime} \operatorname{sign}(\Lambda) P[\Sigma]^{1-\frac{2}{\alpha}}
\end{aligned}
$$

de modo que, se $\alpha=2$, então $\mathbf{V}^{\prime} A \mathbf{V}$ é a identidade com alguns sinais negativos $\operatorname{sign}(\Lambda)$ (se $A$ tiver autovalores negativos) e seu condicionamento é cond $\left(\mathbf{V}^{\prime} A \mathbf{V}\right)=1$. Na última igualdade, todas as as matrizes são diagonais e comutam. Outra observação é que, se $A$ for singular, então $[\Sigma]^{-\frac{1}{\alpha}}$ não está bem definida para qualquer $\alpha$. Nesse caso, dado $\varepsilon>0$ pequeno, pode-se usar uma aproximação do tipo $\left[\Sigma_{+}\right]^{-\frac{1}{\alpha}}$, definido como:

$$
\left[\Sigma_{+}\right]^{-\frac{1}{\alpha}}=\operatorname{diag}\left(\sigma_{i}^{+}\right), \quad \operatorname{com} \quad \sigma_{i}^{+}= \begin{cases}\Sigma_{i i}^{-1 / \alpha}, & \text { se } \Sigma_{i i}^{-1 / \alpha}>\varepsilon \\ \varepsilon, & \text { caso contrário }\end{cases}
$$

Observação 9. A utilização de SVD se justifica porque a dimensão do sistema $\mathbb{S}_{k}$ de todas as instâncias testadas é pequena, em torno de $n=15$. Com isso, seu uso quase não altera o tempo total da execução de uma instância, não acrescentando muito mais do que alguns segundos. Por esse motivo, não será feita uma comparação dos tempos de solução nos resultados computacionais do próximo capítulo. Em aplicações reais deve-se levar em conta a dimensão das matrizes dos sistemas $\mathbb{S}_{k}$ (que é uma matriz de dimensão $m n \times m n$ ) e considerar o tempo da fatoração SVD para essa dimensão.

Outro precondicionador clássico é a diagonal inversa, ou seja, tomar $\mathbf{V}=\operatorname{diag}(|A|)^{-\frac{1}{\alpha}}$, conhecido como Escalonamento Diagonal ou de Jacobi (vide por exemplo Trefethen e Bau III (1997, Lecture 40)). Neste contexto a matriz será usada em valor absoluto por causa do expoente $-1 / \alpha$. 
A escolha $\alpha=2$ talvez seja numericamente ruim, ainda que faça $\operatorname{cond}\left(\mathbf{V}^{\prime} A \mathbf{V}\right)=1$. Com essa formulação, pode-se realizar uma varredura nos valores de $\alpha$ para determinar se outros valores são melhores para essa mudança de base, se 2 ou não. Alguns testes serão feitos para esclarecer essa dúvida.

\subsection{Mudança de base para os ganhos $G_{k}$}

Suponha que se tenha $x \in \mathbb{R}^{n}$ e o controle $u \in \mathbb{R}^{m}$ e portando a matriz de ganho é $G \in \mathbb{R}^{m \times n}$. Do problema variacional, o custo total dado por $\mathbf{J}$ pode ser escrito em função de um ganho $G_{k}$ específico, conforme as equações (A.6) e (A.7). Para simplificar a notação, o índice $k$ será suprimido e o conjunto $\mathscr{C}=\{1, \ldots, m n\}$ será usado.

O custo total $\mathbf{J}$ em função de $G$, dado por $f(G)$, é escrito como:

$$
\mathbf{J}=f(G)=\operatorname{vec}(G)^{\prime}\left[\sum_{i \in \mathscr{N}} X_{i} \otimes Z_{i}\right] \operatorname{vec}(G)+2 \operatorname{vec}\left(\sum_{i \in \mathscr{N}} M_{i}^{\prime} X_{i}\right)^{\prime} \operatorname{vec}(G)+c,
$$

em que $c$ é o somatório dos custos que dependem apenas dos demais ganhos $\left\{G_{j}\right\}_{j \neq k}$, e não deste $G_{k}$ especificamente.

Dada uma base de matrizes $\mathbf{K}=\left\{K_{i} \mid K_{i} \in \mathbb{R}^{m \times n} \forall i \in \mathscr{C}\right\}$ que possa gerar a matriz $G$ (as matrizes $K_{i}$ são linearmente independentes), pode-se reescrever $G$ nessa nova base, conforme abaixo:

$$
G=a_{1} K_{1}+a_{2} K_{2}+\ldots+a_{m n} K_{m n}=\sum_{k \in \mathscr{C}} a_{k} K_{k}, \quad a_{k} \in \mathbb{R},
$$

ou matricialmente, com $I_{n}$ e $I_{m}$ sendo a matriz identidade de ordem $n$ e $m$ respectivamente:

$$
G \stackrel{(4.4)}{=} \underbrace{\left[\begin{array}{llll}
K_{1} & K_{2} & \ldots & K_{m n}
\end{array}\right]}_{\mathbf{K}}\left[\begin{array}{c}
a_{1} I_{n} \\
a_{2} I_{n} \\
\vdots \\
a_{m n} I_{n}
\end{array}\right]=\left[\begin{array}{llll}
a_{1} I_{m} & a_{2} I_{m} & \ldots & a_{m n} I_{m}
\end{array}\right] \underbrace{\left[\begin{array}{c}
K_{1} \\
K_{2} \\
\vdots \\
K_{m n}
\end{array}\right]}_{\mathbf{K}},
$$

e então a forma vetorizada desse ganho $G$ é:

$$
\operatorname{vec}(G) \stackrel{(4.5)}{=} \underbrace{\left[\begin{array}{lll}
\operatorname{vec}\left(K_{1}\right) & \ldots & \operatorname{vec}\left(K_{m n}\right)
\end{array}\right]}_{\mathbf{V}} \vec{a}=\mathbf{V} \vec{a},
$$

em que $\mathbf{V}$ é a base de matrizes $\mathbf{K}$ com seus elementos vetorizados, e o vetor $\vec{a} \in \mathbb{R}^{m n \times 1}$ é dado por $\vec{a}=\left(a_{1}, \ldots, a_{m n}\right)^{\prime}$.

Com essa mudança de base, pode-se reescrever a Equação 4.3 em termos de $\vec{a}$ usando a nova base em (4.6):

Exemplo: se $m=3, n=2$, então $\mathscr{C}=\{1,2,3,4,5,6\}$.

A base $\mathbf{K}$ é uma base para a matriz $G$, enquanto que a base $\mathbf{V}$ é uma base para o vetor $\mathbf{v e c}(G)$. 


$$
f(G)=f(\vec{a})=\vec{a}^{\prime} \mathbf{V}^{\prime}\left[\sum_{i \in \mathscr{N}} X_{i} \otimes Z_{i}\right] \mathbf{V} \vec{a}+\vec{a}^{\prime} \mathbf{V}^{\prime} \mathbf{v e c}\left(\sum_{i \in \mathscr{N}} 2 M_{i}^{\prime} X_{i}\right)+c .
$$

Dessa forma, derivando a expressão acima em relação a $\vec{a}$ e igualando a zero, tem-se o seguinte sistema linear:

$$
\mathbf{V}^{\prime}\left[\sum_{i \in \mathscr{N}} X_{i} \otimes Z_{i}\right] \mathbf{V} \vec{a}=\mathbf{V}^{\prime} \mathbf{v e c}\left(-\sum_{i \in \mathscr{N}} M_{i}^{\prime} X_{i}\right),
$$

onde a solução desejada para o ganho é

$$
\operatorname{vec}(G) \stackrel{(4.6)}{=} \mathbf{V} \vec{a} .
$$

\subsection{Mudança de base para os ganhos $G_{k}$ : segunda formu- lação}

Sendo $x \in \mathbb{R}^{n}$ e $u \in \mathbb{R}^{m}$, e portanto $G \in \mathbb{R}^{m \times n}$, da Equação A.6, tem-se

$$
\mathbf{J}=f(G)=c+\underbrace{\sum_{i \in \mathscr{N}} \operatorname{tr}\left(X_{i} G^{\prime} Z_{i} G\right)}_{\phi}+\underbrace{\sum_{i \in \mathscr{N}} 2 \operatorname{tr}\left(X_{i} M_{i} G\right)}_{\psi} .
$$

Para simplificar notação, define-se o conjunto $\mathscr{C}=\{1, \ldots, m n\}$. Da Equação 4.4, escrevendo $G$ em uma nova base $\mathbf{K}=\left\{K_{i} \mid K_{i} \in \mathbb{R}^{m \times n} \forall i \in \mathscr{C}\right\}$, em que as matrizes $K_{i}$ são linearmente independentes, vem

$$
G=a_{1} K_{1}+a_{2} K_{2}+\ldots+a_{m n} K_{m n}=\sum_{k \in \mathscr{C}} a_{k} K_{k}, \quad a_{k} \in \mathbb{R}
$$

Com essa nova representação do ganho $G$ serão feitas contas com $m=1$ e $n=3$, mas o processo será generalizado para quaisquer $m$ e $n$ nas linhas seguintes.

Inicialmente, analisa-se a parcela $\phi$ :

$$
\begin{aligned}
\phi=\sum_{i \in \mathscr{N}} \operatorname{tr} & {\left[X_{i}\left(\sum_{k=1}^{3} a_{k} K_{k}\right)^{\prime} Z_{i}\left(\sum_{k=1}^{3} a_{k} K_{k}\right)\right] } \\
=\sum_{i \in \mathscr{N}} \operatorname{tr} & {\left[X_{i}\left(a_{1} K_{1}+a_{2} K_{2}+a_{3} K_{3}\right)^{\prime} Z_{i}\left(a_{1} K_{1}+a_{2} K_{2}+a_{3} K_{3}\right)\right] } \\
=\sum_{i \in \mathscr{N}} \operatorname{tr}[ & X_{i}\left(a_{1} a_{1} K_{1}^{\prime} Z_{i} K_{1}+a_{1} a_{2} K_{1}^{\prime} Z_{i} K_{2}+a_{1} a_{3} K_{1}^{\prime} Z_{i} K_{3}+\right. \\
& +a_{2} a_{1} K_{2}^{\prime} Z_{i} K_{1}+a_{2} a_{2} K_{2}^{\prime} Z_{i} K_{2}+a_{2} a_{3} K_{2}^{\prime} Z_{i} K_{3}+ \\
& \left.\left.+a_{3} a_{1} K_{3}^{\prime} Z_{i} K_{1}+a_{3} a_{2} K_{3}^{\prime} Z_{i} K_{2}+a_{3} a_{3} K_{3}^{\prime} Z_{i} K_{3}\right)\right]
\end{aligned}
$$

Exemplo: se $m=3, n=2$, então $\mathscr{C}=\{1,2,3,4,5,6\}$. 
Escolhendo um índice $p \in \mathscr{C}$ (por exemplo $p=1$, como ilustrado em vermelho em (??)), destacando a parte de $\phi$ que depende de $a_{p}$, deixando as demais parcelas como constante $c_{i p}$ :

$$
\phi=\phi\left(a_{p}\right)=\sum_{i \in \mathscr{N}}\left[c_{i p}+a_{p}^{2} \operatorname{tr}\left(X_{i} K_{p}^{\prime} Z_{i} K_{p}\right)+2 a_{p} \sum_{\substack{q \in \mathscr{C} \\ q \neq p}} a_{q} \operatorname{tr}\left(X_{i} K_{p}^{\prime} Z_{i} K_{q}\right)\right]
$$

de modo que sua derivada em relação a $a_{p}$ é

$$
\begin{aligned}
\frac{d \phi\left(a_{p}\right)}{d a_{p}} & =\sum_{i \in \mathscr{N}}\left[2 a_{p} \operatorname{tr}\left(X_{i} K_{p}^{\prime} Z_{i} K_{p}\right)+2 \sum_{\substack{q \in \mathscr{C} \\
q \neq p}} a_{q} \operatorname{tr}\left(X_{i} K_{p}^{\prime} Z_{i} K_{q}\right)\right] \\
& =\sum_{i \in \mathscr{N}} \sum_{q \in \mathscr{C}} 2 a_{q} \operatorname{tr}\left(X_{i} K_{p}^{\prime} Z_{i} K_{q}\right) .
\end{aligned}
$$

Agora fazendo as contas para a parcela $\psi$, vem:

$$
\psi=\psi\left(a_{p}\right)=\sum_{i \in \mathscr{N}} 2 \operatorname{tr}\left[X_{i} M_{i}\left(a_{p} K_{p}+\sum_{\substack{q \in \mathscr{C} \\ q \neq p}} a_{q} K_{q}\right)\right]=\sum_{i \in \mathscr{N}} 2 a_{p} \operatorname{tr}\left(X_{i} M_{i} K_{p}\right)+\sum_{\substack{q \in \mathscr{C} \\ q \neq p}} 2 a_{q} \operatorname{tr}\left(X_{i} M_{i} K_{q}\right) .
$$

Escolhido um índice $p \in \mathscr{C}$, derivando $\psi$ em relação a $a_{p}$, tem-se

$$
\frac{d \psi\left(a_{p}\right)}{d a_{p}}=\sum_{i \in \mathscr{N}} 2 \operatorname{tr}\left(X_{i} M_{i} K_{p}\right)
$$

Voltando à expressão de $f(G)$,

$$
\frac{d f}{d \vec{a}}=\frac{d \phi}{d \vec{a}}+\frac{d \psi}{d \vec{a}}
$$

em que $\vec{a}=\left(a_{1}, \ldots, a_{m n}\right)^{\prime}$, de forma que, para cada $p \in \mathscr{C}$ se tem

$$
\frac{\partial f}{\partial a_{p}}=\frac{\partial \phi}{\partial a_{p}}+\frac{\partial \psi}{\partial a_{p}}
$$

Fazendo o gradiente de $f$ igual a zero, fica

$$
\begin{gathered}
\frac{\partial \phi}{\partial a_{p}}=-\frac{\partial \psi}{\partial a_{p}}, \quad \forall p \in \mathscr{C}, \\
(4.9)(4.10) \Longrightarrow 2 \sum_{i \in \mathscr{N}} \sum_{q \in \mathscr{C}} a_{q} \operatorname{tr}\left(X_{i} K_{p}^{\prime} Z_{i} K_{q}\right)=-\sum_{i \in \mathscr{N}} 2 \operatorname{tr}\left(X_{i} M_{i} K_{p}\right), \quad \forall p \in \mathscr{C},
\end{gathered}
$$

e isso dá o seguinte sistema linear:

$$
\sum_{i \in \mathscr{N}}\left[\begin{array}{cccc}
\operatorname{tr}\left(X_{i} K_{1}^{\prime} Z_{i} K_{1}\right) & \operatorname{tr}\left(X_{i} K_{1}^{\prime} Z_{i} K_{2}\right) & \ldots & \operatorname{tr}\left(X_{i} K_{1}^{\prime} Z_{i} K_{m n}\right) \\
\operatorname{tr}\left(X_{i} K_{2}^{\prime} Z_{i} K_{1}\right) & \operatorname{tr}\left(X_{i} K_{2}^{\prime} Z_{i} K_{2}\right) & \ldots & \operatorname{tr}\left(X_{i} K_{2}^{\prime} Z_{i} K_{m n}\right) \\
: & : & \ldots & : \\
\operatorname{tr}\left(X_{i} K_{m n}^{\prime} Z_{i} K_{1}\right) & \operatorname{tr}\left(X_{i} K_{m n}^{\prime} Z_{i} K_{2}\right) & \ldots & \operatorname{tr}\left(X_{i} K_{m n}^{\prime} Z_{i} K_{m n}\right)
\end{array}\right]\left[\begin{array}{c}
a_{1} \\
a_{2} \\
: \\
a_{m n}
\end{array}\right]=-\sum_{i \in \mathscr{N}}\left[\begin{array}{c}
\operatorname{tr}\left(X_{i} M_{i} K_{1}\right) \\
\operatorname{tr}\left(X_{i} M_{i} K_{2}\right) \\
: \\
\operatorname{tr}\left(X_{i} M_{i} K_{m n}\right)
\end{array}\right]
$$


Se for definido $W_{i}=X_{i} \otimes Z_{i}$ e $\vec{K}_{p}=\operatorname{vec}\left(K_{p}\right)$, o sistema acima pode ser reescrito como

$$
\sum_{i \in \mathscr{N}}\left[\begin{array}{cccc}
\vec{K}_{1}^{\prime} W_{i} \vec{K}_{1} & \vec{K}_{1}^{\prime} W_{i} \vec{K}_{2} & \ldots & \vec{K}_{1}^{\prime} W_{i} \vec{K}_{m n} \\
\vec{K}_{2}^{\prime} W_{i} \vec{K}_{1} & \vec{K}_{2}^{\prime} W_{i} \vec{K}_{2} & \ldots & \vec{K}_{2}^{\prime} W_{i} \vec{K}_{m n} \\
: & : & \ldots & : \\
\vec{K}_{m n}^{\prime} W_{i} \vec{K}_{1} & \vec{K}_{m n}^{\prime} W_{i} \vec{K}_{2} & \ldots & \vec{K}_{m n}^{\prime} W_{i} \vec{K}_{m n}
\end{array}\right]\left[\begin{array}{c}
a_{1} \\
a_{2} \\
: \\
a_{m n}
\end{array}\right]=-\sum_{i \in \mathscr{N}}\left[\begin{array}{c}
\operatorname{tr}\left(X_{i} M_{i} K_{1}\right) \\
\operatorname{tr}\left(X_{i} M_{i} K_{2}\right) \\
: \\
\operatorname{tr}\left(X_{i} M_{i} K_{m n}\right)
\end{array}\right]
$$

Ou ainda, vetorizando o segundo membro ao se definir $Y_{i}=M_{i}^{\prime} X_{i}$ e $\vec{Y}_{i}=\operatorname{vec}\left(Y_{i}\right)$, o sistema finalmente chega a

$$
\sum_{i \in \mathscr{N}}\left[\begin{array}{cccc}
\vec{K}_{1}^{\prime} W_{i} \vec{K}_{1} & \vec{K}_{1}^{\prime} W_{i} \vec{K}_{2} & \ldots & \vec{K}_{1}^{\prime} W_{i} \vec{K}_{m n} \\
\vec{K}_{2}^{\prime} W_{i} \vec{K}_{1} & \vec{K}_{2}^{\prime} W_{i} \vec{K}_{2} & \ldots & \vec{K}_{2}^{\prime} W_{i} \vec{K}_{m n} \\
: & : & \ldots & : \\
\vec{K}_{m n}^{\prime} W_{i} \vec{K}_{1} & \vec{K}_{m n}^{\prime} W_{i} \vec{K}_{2} & \ldots & \vec{K}_{m n}^{\prime} W_{i} \vec{K}_{m n}
\end{array}\right]\left[\begin{array}{c}
a_{1} \\
a_{2} \\
: \\
a_{m n}
\end{array}\right]=-\sum_{i \in \mathscr{N}}\left[\begin{array}{c}
\vec{Y}_{i}^{\prime} \vec{K}_{1} \\
\vec{Y}_{i}^{\prime} \vec{K}_{2} \\
\vdots \\
\vec{Y}_{i}^{\prime} \vec{K}_{m n}
\end{array}\right]
$$

Conclui-se assim essa segunda formulação da mudança de base

$$
G=\sum_{k \in \mathscr{C}} a_{k} K_{k}
$$

Um ponto importante, que justifica as estratégias de precondicionamento utilizadas neste trabalho, é que a ordem de precedência entre os operandos é numericamente importante, ainda que matematicamente sejam equivalentes. Trata-se do somatório na Equação 4.7. Se o somatório for executado antes de se fazer a mudança de base, a solução é numericamente pior em relação àquela obtida fazendo-se primeiro a mudança de base. Esse fenômeno incentivou a busca por formulações alternativas para a mudança de base, como por exemplo na Equação 4.12 e na Equação 4.13. Com essas premissas, fazem-se as seguintes definições das técnicas de precondicionamento.

Definição 7 (Auxiliares). No intuito de não repetir definições, além de esclarecer a notação

Veja como foi feito para que $\operatorname{tr}\left(X_{i} G^{\prime} Z_{i} G\right)=\operatorname{vec}(G)^{\prime}\left[X_{i} \otimes Z_{i}\right] \operatorname{vec}(G)$ na Equação A.6 e Equação A.7, bem como $\operatorname{tr}\left(X_{i} M_{i} G\right)=\operatorname{vec}\left(M_{i}^{\prime} X_{i}\right)^{\prime} \operatorname{vec}(G)$. 
utilizada, defina

$$
\left\{\begin{array}{l}
W_{i}=X_{i} \otimes Z_{i} \in \mathbb{R}^{m n \times m n}, \quad \forall i \in \mathscr{N} \\
\sum_{i \in \mathscr{N}} W_{i} \stackrel{S V D}{=} U \Sigma V^{\prime} \\
\vec{K}_{k}=\mathbf{v e c}\left(K_{k}\right), \quad K_{k} \in \mathbb{R}^{m \times n} \forall k=1, \ldots, m n \\
\mathbf{V}(\alpha) \stackrel{(4.2)}{=} U\left[\Sigma_{+}\right]^{-\frac{1}{\alpha}}=\left[\vec{K}_{1}\left|\vec{K}_{2}\right| \ldots \mid \vec{K}_{m n}\right]=(\text { matriz) } \\
\mathbf{v e c}(G)=\mathbf{V}(\alpha) \vec{a}=(\text { solução) } \\
G=\sum_{k=1}^{m n} K_{k} a_{k}=(\text { solução) } \\
\vec{a}=\left(a_{1}, \ldots, a_{m n}\right)^{\prime} \\
\mathbf{K}(\alpha)=\left\{K_{k}=\mathbf{v e c}_{m, n}^{-1}\left(\mathbf{V}(\alpha)_{\text {coluna }=k}\right)\right\}=\text { (coleção de matrizes) } \\
\overrightarrow{\mathbf{K}}(\alpha)=\left\{\vec{K}_{k}=\mathbf{V}(\alpha)_{\text {coluna }=k}\right\}=(\text { coleção de vetores) }
\end{array}\right.
$$

O sistema linear $\mathbb{S}_{k}$ dado na Equação 3.11 será reescrito aplicando-se precondicionadores. O novo sistema linear $\widetilde{\mathbb{S}}_{k}$ possibilitará obter as coordenadas $\vec{a}$ da solução $G$ na nova base $\mathbf{V}, \mathbf{K}$ ou $\overrightarrow{\mathbf{K}}$. Para recuperar a solução $G$, basta utilizar a Equação 4 .14e e recuperar a matriz por meio de $G=\operatorname{vec}_{m, n}^{-1}(\operatorname{vec}(G))$ ou então diretamente somando as matrizes pela Equação 4.14f. Assim, o objetivo agora é obter as coordenadas de $G$ na nova base, e não mais $G$.

Definição 8 (Precondicionador 1). A mudança de base em $\mathbf{V}(\alpha)$ da Equação 4.7 tem como preferência o operador somatório, usando a Equação 4.14d:

$$
\left[\mathbf{V}(\alpha)^{\prime}\left[\sum_{i \in \mathscr{N}} W_{i}\right] \mathbf{V}(\alpha)\right] \vec{a}=\mathbf{V}(\alpha)^{\prime} \operatorname{vec}\left(-\sum_{i \in \mathscr{N}} M_{i}^{\prime} X_{i}\right)
$$

Definição 9 (Precondicionador 2). A mudança de base em $\mathbf{V}(\alpha)$ (base de vetores) da Equação 4.7 tem a preferência diante do operador somatório, com a Equação 4.14d.

$$
\left[\sum_{i \in \mathscr{N}} \mathbf{V}(\alpha)^{\prime} W_{i} \mathbf{V}(\alpha)\right] \vec{a}=\mathbf{V}(\alpha)^{\prime} \mathbf{v e c}\left(-\sum_{i \in \mathscr{N}} M_{i}^{\prime} X_{i}\right)
$$

Definição 10 (Precondicionador 3). A mudança de base em $\mathbf{K}(\alpha)$ (base de matrizes) usando o operador traço explicitamente, conforme a Equação 4.11, usando a Equação 4.14h.

$$
\sum_{i \in \mathscr{N}}\left[\begin{array}{ccc}
\operatorname{tr}\left(X_{i} K_{1}^{\prime} Z_{i} K_{1}\right) & \ldots & \operatorname{tr}\left(X_{i} K_{1}^{\prime} Z_{i} K_{m n}\right) \\
\vdots & \ldots & : \\
\operatorname{tr}\left(X_{i} K_{m n}^{\prime} Z_{i} K_{1}\right) & \ldots & \operatorname{tr}\left(X_{i} K_{m n}^{\prime} Z_{i} K_{m n}\right)
\end{array}\right]\left[\begin{array}{c}
a_{1} \\
: \\
a_{m n}
\end{array}\right]=-\sum_{i \in \mathscr{N}}\left[\begin{array}{c}
\operatorname{tr}\left(X_{i} M_{i} K_{1}\right) \\
: \\
\operatorname{tr}\left(X_{i} M_{i} K_{m n}\right)
\end{array}\right]
$$

Definição 11 (Precondicionador 4). A mudança de base em $\overrightarrow{\mathbf{K}}(\alpha)$ e $\mathbf{K}(\alpha)$ usando o operador traço implicitamente, conforme a Equação 4.12, com as equações Equação 4.14h e Equação 4.14i. 


$$
\sum_{i \in \mathscr{N}}\left[\begin{array}{ccc}
\vec{K}_{1}^{\prime} W_{i} \vec{K}_{1} & \ldots & \vec{K}_{1}^{\prime} W_{i} \vec{K}_{m n} \\
: & \ldots & : \\
\vec{K}_{m n}^{\prime} W_{i} \vec{K}_{1} & \ldots & \vec{K}_{m n}^{\prime} W_{i} \vec{K}_{m n}
\end{array}\right]\left[\begin{array}{c}
a_{1} \\
: \\
a_{m n}
\end{array}\right]=-\sum_{i \in \mathscr{N}}\left[\begin{array}{c}
\operatorname{tr}\left(X_{i} M_{i} K_{1}\right) \\
: \\
\operatorname{tr}\left(X_{i} M_{i} K_{m n}\right)
\end{array}\right]
$$

Definição 12 (Precondicionador 5). A mudança de base não é fundamentada na SVD, mas sim no Escalonamento de Jacobi, usando em seguida a ideia da Definição 9.

$$
\begin{gathered}
\mathbf{V}(\alpha)=\left|\operatorname{diag}\left(\sum_{i \in \mathscr{N}} W_{i}\right)\right|^{-\frac{1}{\alpha}} . \\
{\left[\sum_{i \in \mathscr{N}} \mathbf{V}(\alpha)^{\prime} W_{i} \mathbf{V}(\alpha)\right] \vec{a}=\mathbf{V}(\alpha)^{\prime} \operatorname{vec}\left(-\sum_{i \in \mathscr{N}} M_{i}^{\prime} X_{i}\right)}
\end{gathered}
$$

Esses precondicionadores serão chamados simplesmente de P1, P2, P3, P4 e P5 respectivamente. Em contraste, quando não houver precondicionador, será referido por P0.

\subsection{Máscaras}

A pseudoinversa $A^{+}$de uma dada matriz $A$, bastante conhecida na literatura de álgebra linear aplicada, como em Trefethen e Bau III (1997, Lecture 11) por exemplo, é dada pela seguinte expressão:

$$
A^{+}=\left(A^{\prime} A\right)^{-1} A^{\prime}
$$

Ela tem a propriedade de "resolver" o sistema linear $A x=b$ minimizando o resíduo $\|r\|=\| A x-$ $b \|$ (problema de mínimos quadrados). Se $A$ não é singular, então $A^{+}=A^{-1}$. Uma propriedade especial da pseudoinversa é que o espaço nulo (Petersen (2012, Proposition 4.9.2))

$$
\operatorname{núcleo}(A)=\operatorname{núcleo}\left(A^{+}\right)=\text {núcleo }\left(A^{+} A\right)
$$

Definição 13. Dadas duas matrizes arbitrárias de mesma dimensão, definem-se as máscaras

$$
\operatorname{mask}(P, Q)=\left(Q Q^{+}\right)^{\prime} P\left(Q Q^{+}\right)
$$

Definição 14 (Espaço Nulo). Define-se o espaço nulo de um operador linear $L \in \mathbb{V}_{n \times n}^{\mathscr{N}} \rightarrow \mathbb{V}_{n \times n}^{\mathcal{N}}$ com relação ao produto interno $\langle\cdot, \cdot\rangle$, denotado por núcleo* $(L)$, como sendo a reunião de todos os pontos $X \in \mathbb{V}_{n \times n}^{\mathscr{N}}$ tais que

$$
\langle X, L\rangle=0
$$

Como o produto interno é comutativo, isso significa que $L$ também está no espaço nulo de $X$.

Lema 1. Sejam $X, L \in \mathbb{V}_{n \times n}^{\mathscr{N}}$ simétricos e positivos semidefinidos. Assim, $\operatorname{tr}\left(X_{i} L_{i}\right)=0$ para algum $i \in \mathscr{N}$ se, e somente se $X_{i} L_{i}=0$. Dessa forma, $\langle X, L\rangle=0$ se, e somente se $X L=0$. 
Demonstração. $(\Rightarrow)$ Inicialmente, como $X_{i}, L_{i}$ são simétricos e positivos semidefinidos, existem suas decomposições espectrais (vide Nocedal e Wright (2006, p. 597))

$$
X_{i}=\sum_{r=1}^{p} \alpha_{r} x_{r} x_{r}^{\prime} \quad \text { e } \quad L_{i}=\sum_{s=1}^{p} \beta_{s} \ell_{s} \ell_{s}^{\prime}, \quad \operatorname{com} x_{r}, \ell_{s} \in \mathbb{R}^{n} \forall r, s \in\{1, \ldots, n\}, \alpha_{r}, \beta_{s} \in \mathbb{R}, i \in \mathscr{N},
$$

em que $\alpha_{r}, \beta_{s} \geq 0 \forall r, s \in\{1, \ldots, n\}$ são os autovalores de $X_{i}$ e $L_{i}$ respectivamente. Assim,

$$
\begin{aligned}
0=\operatorname{tr}\left(X_{i} L_{i}\right) & =\operatorname{tr}\left(\sum_{r=1}^{n} \alpha_{r} x_{r} x_{r}^{\prime} \sum_{s=1}^{n} \beta_{s} \ell_{s} \ell_{s}^{\prime}\right)=\sum_{r=1}^{n} \sum_{s=1}^{n} \alpha_{r} \beta_{s} \operatorname{tr}\left(x_{r} x_{r}^{\prime} \ell_{s} \ell_{s}^{\prime}\right) \\
& =\sum_{r=1}^{n} \sum_{s=1}^{n} \alpha_{r} \beta_{s} \operatorname{tr}\left(x_{r}^{\prime} \ell_{s} \ell_{s}^{\prime} x_{r}\right)=\sum_{r=1}^{n} \sum_{s=1}^{n} \alpha_{r} \beta_{s}\left(x_{r}^{\prime} \ell_{s}\right)^{2} .
\end{aligned}
$$

Isso mostra que, para cada par $(r, s)$ pelo menos uma das seguintes coisas acontece: ou $\alpha_{r}=0$, ou $\beta_{s}=0$ ou $x_{r}^{\prime} \ell_{s}=0$. Como isso acontece para todos os pares $(r, s)$, segue o resultado

$$
X_{i} L_{i}=\sum_{r=1}^{n} \sum_{s=1}^{n} \alpha_{r} \beta_{s} x_{r} x_{r}^{\prime} \ell_{s} \ell_{s}^{\prime}=0
$$

$(\Leftarrow)$ Imediatamente se observa que, se $X_{i} L_{i}=0$ então $\operatorname{tr}\left(X_{i} L_{i}\right)=0$. A segunda parte é imediata, pois se a soma de coisas não negativas é zero, então cada uma delas é zero.

Proposição 2. Sejam $X, L \in \mathbb{V}_{n \times n}^{\mathscr{N}}$ dados pelo Definição 2 e pelo Definição 3 no modelo sem ruído, isto é, $H=0$. Denote por $\mathbf{L}_{0}^{k}=\left.L^{k}\right|_{\left\{G_{r}=0, k \leq r<T\right\}}$. Assim, se para um dado $k \in \mathscr{H}$ acontecer $\left\langle X^{k}, L^{k}\right\rangle=0$, onde é utilizado um ganho $G_{k}$, então:

1. $G_{k} X^{k}=0 ; C X^{k}=0$;

2. $\left.X^{k+1}\right|_{G_{k} \neq 0}=\left.X^{k+1}\right|_{\left\{G_{k}=0\right\}}$;

3. $\left\langle X^{k+t}, L^{k+t}\right\rangle=0 ; 0<t<T$ se não houver ruído aditivo;

Demonstração. (1) Pelas hipóteses, tem-se que $L^{k}=C+G_{k}^{\prime} \bullet D \bullet G_{k}+\mathscr{L}_{\overline{\mathbf{A}}^{k}}\left(L^{k+1}\right)$, em que $C=C^{\prime} \geq 0$ e $D=D^{\prime}>0$. Assim,

$$
0=\left\langle X^{k}, L^{k}\right\rangle \geq\left\langle X^{k}, G_{k}^{\prime} \bullet D \bullet G_{k}\right\rangle+\left\langle X^{k}, C\right\rangle \geq 0 .
$$

Pelo Lema 1 , tem-se que $G_{k} X_{i}=0 \forall i \in \mathscr{N}$, pois $\left\langle X^{k}, G_{k}^{\prime} \bullet D \bullet G_{k}\right\rangle=\left\langle G_{k}^{\prime}, D \bullet G_{k} X^{k}\right\rangle=0$ e $D>0$. Também $C X^{k}=0$ pelo mesmo lema.

(2) Pela definição de $X$ no Definição 2,

$$
X_{i}^{k+1}=\sum_{j \in \mathscr{N}} \mathbb{P}_{j i}\left(A_{j}+B_{j} G_{k}\right) X_{j}^{k}\left(A_{j}+B_{j} G_{k}\right)^{\prime} .
$$

Por (1), isso se reduz à fórmula sem o ganho $G_{k}$

$$
X_{i}^{k+1}=\sum_{j \in \mathscr{N}} \mathbb{P}_{j i}\left(A_{j}\right) X_{j}^{k}\left(A_{j}\right)^{\prime} .
$$


(3) Pela definição dos custos de continuação (caso sem ruído):

$$
0=\left.J\right|_{k} ^{T-1}=\left\langle X^{k}, L^{k}\right\rangle \geq\left\langle X^{k+t}, L^{k+t}\right\rangle=\left.J\right|_{k+t} ^{T-1} \geq 0 .
$$

Lema 2. Denote por $\mathbf{L}_{0}^{k}=\left.L^{k}\right|_{\left\{G_{r}=0, k \leq r<T\right\}}$, conforme o Definição 3. Então, para qualquer $L^{k}$ dado pelas mesmas equações,

$$
\text { núcleo*}^{*}\left(L^{k}\right) \subseteq \text { núcleo*}^{*}\left(\mathbf{L}_{0}^{k}\right)
$$

Demonstração. A prova será por indução. Inicialmente, note que ambos $L$ e $\mathbf{L}_{0}$ iniciam no mesmo ponto $L^{T}=0$. Agora, suponha que, para qualquer ganho $G_{k}$ dado seja válido

$$
\begin{aligned}
L_{i}^{k} & =C_{i}+G_{k}^{\prime} D_{i} G_{k}+\mathscr{L}_{\left(A+B G_{k}\right)}^{i}\left(L^{k+1}\right)=C_{i}+G_{k}^{\prime} D_{i} G_{k}+\sum_{j \in \mathscr{N}} \mathbb{P}_{i j}\left(A_{i}+B_{i} G_{k}\right)^{\prime} L_{j}^{k+1}\left(A_{i}+B_{i} G_{k}\right) \\
& =C_{i}+G_{k}^{\prime} D_{i} G_{k}+\mathscr{L}_{A}^{i}\left(\mathbf{L}_{0}^{k+1}\right)+\sum_{j \in \mathscr{N}} \mathbb{P}_{i j}\left(G_{k}^{\prime} B_{i}^{\prime} L_{j}^{k+1} A_{i}+A_{i}^{\prime} L_{j}^{k+1} B_{i} G_{k}+G_{k}^{\prime} B_{i}^{\prime} L_{j}^{k+1} B_{i} G_{k}\right) \\
& =\mathbf{L}_{0 i}^{k}+\underbrace{G_{k}^{\prime} D_{i} G_{k}+\sum_{j \in \mathscr{N}} \mathbb{P}_{i j}\left(G_{k}^{\prime} B_{i}^{\prime} L_{j}^{k+1} A_{i}+A_{i}^{\prime} L_{j}^{k+1} B_{i} G_{k}+G_{k}^{\prime} B_{i}^{\prime} L_{j}^{k+1} B_{i} G_{k}\right)}_{F_{i}^{k}} . \\
& =\mathbf{L}_{0 i}^{k}+F_{i}^{k},
\end{aligned}
$$

em que $F_{i}^{k}$ é simétrica positiva semidefinida. Então

$$
\begin{aligned}
& L_{i}^{k-1}=C_{i}+G_{k-1}^{\prime} D_{i} G_{k-1}+\mathscr{L}_{\left(A+B G_{k-1}\right)}^{i}\left(L^{k}\right) \\
& =C_{i}+G_{k-1}^{\prime} D_{i} G_{k-1}+\sum_{j \in \mathscr{N}} \mathbb{P}_{i j}\left(A_{i}+B_{i} G_{k-1}\right)^{\prime} L_{j}^{k}\left(A_{i}+B_{i} G_{k-1}\right) \\
& =C_{i}+G_{k-1}^{\prime} D_{i} G_{k-1}+\sum_{j \in \mathscr{N}} \mathbb{P}_{i j}\left(A_{i}+B_{i} G_{k-1}\right)^{\prime}\left[\mathbf{L}_{0 j}^{k}+F_{j}^{k}\right]\left(A_{i}+B_{i} G_{k-1}\right) \\
& =C_{i}+\mathscr{L}_{A}^{i}\left(\mathbf{L}_{0}^{k}\right)+\underbrace{G_{k-1}^{\prime} D_{i} G_{k-1}+\mathscr{L}_{\left(A+B G_{k-1}\right)}^{i}\left(F^{k}\right)+\sum_{j \in \mathscr{N}} \mathbb{P}_{i j}\left(G_{k-1}^{\prime} B_{i}^{\prime} \mathbf{L}_{0 j}^{k} A_{i}+A_{i}^{\prime} \mathbf{L}_{0 j}^{k} B_{i} G_{k-1}+\ldots\right.}_{F_{i}^{k-1} \ldots} \\
& \quad \ldots+\underbrace{\left.G_{k-1}^{\prime} B_{i}^{\prime} \mathbf{L}_{0 j}^{k} B_{i} G_{k-1}\right)}_{\ldots F_{i}^{k-1}} \\
& =\mathbf{L}_{0 i}^{k-1}+F_{i}^{k-1} .
\end{aligned}
$$

Ao se fazer mais um passo indutivo análogo, consegue-se a demonstração. Dessa forma, para qualquer $X^{k} \in \mathbb{V}_{n \times n}^{\mathscr{N}}$ simétrico e positivo semidefinido, se tem

$$
0=\left\langle X^{k}, L^{k}\right\rangle \geq\left\langle X^{k}, \mathbf{L}_{0}^{k}\right\rangle \geq 0
$$

e portanto núcleo* $\left(L^{k}\right) \subseteq$ núcleo*$^{*}\left(\mathbf{L}_{0}^{k}\right)$ para todo $k \in \mathscr{H}$. 
A propriedade do Lema 2 às vezes pode ser perdida por erros numéricos ao longo das iterações do MV. Uma forma de melhorar isso é aplicando as máscaras definidas na Equação 4.22, conforme o lema a seguir.

Lema 3 (Espaço nulo). Suponha que erros numéricos acabem por contaminar $L$, dada pelo Definição 3 para algum $G$, passando a ser $\widehat{L}=L+\delta L$, com $\delta L>0$ simétrico positivo definido. Considere $\mathbf{L}_{0}=\left.L^{k}\right|_{\left\{G_{r}=0, k \leq r<T\right\}} \in \mathbb{V}_{n \times n}^{\mathscr{N}}$. Assim,

$$
\operatorname{núcleo}^{*}\left(\operatorname{mask}\left(\widehat{L}, \mathbf{L}_{0}\right)\right)=\operatorname{núcleo}^{*}\left(\mathbf{L}_{0}\right) .
$$

Demonstração. Considere $L+\delta L$ agora simétrica e positiva definida. Então

$$
\begin{aligned}
0 & =\left\langle X,\left(\mathbf{L}_{0}^{+} \mathbf{L}_{0}\right)^{\prime}(L+\delta L)\left(\mathbf{L}_{0}^{+} \mathbf{L}_{0}\right)\right\rangle \\
& =\left\langle\left(\mathbf{L}_{0}^{+} \mathbf{L}_{0}\right) X\left(\mathbf{L}_{0}^{+} \mathbf{L}_{0}\right)^{\prime}, L\right\rangle+\left\langle\left(\mathbf{L}_{0}^{+} \mathbf{L}_{0}\right) X\left(\mathbf{L}_{0}^{+} \mathbf{L}_{0}\right)^{\prime}, \delta L\right\rangle .
\end{aligned}
$$

Como $\delta L>0$, então, pelo Lema 1 , núcleo* $(\delta L)=0$ e então $X \in$ núcleo* $^{*}\left(\mathbf{L}_{0}^{+} \mathbf{L}_{0}\right)$. Mas pela Equação $4.21, X \in$ núcleo* $^{*}\left(\mathbf{L}_{0}\right)$. Assim, núcleo* $\left(\operatorname{mask}\left(\widehat{L}, L_{0}\right)\right) \subseteq$ núcleo*$^{*}\left(\mathbf{L}_{0}\right)$. Por outro lado, se $X \in$ núcleo* $^{*}\left(\mathbf{L}_{0}\right)$, então imediatamente se tem pelo Lema 1 que

$$
\left\langle X,\left(\mathbf{L}_{0}^{+} \mathbf{L}_{0}\right)^{\prime}(L+\delta L)\left(\mathbf{L}_{0}^{+} \mathbf{L}_{0}\right)\right\rangle=\left\langle\left(\mathbf{L}_{0}^{+} \mathbf{L}_{0}\right)^{\prime},(L+\delta L)\left(\mathbf{L}_{0}^{+} \mathbf{L}_{0}\right) X\right\rangle=0 .
$$

Com isso, $\operatorname{núcleo*}^{*}\left(\mathbf{L}_{0}\right) \subseteq \operatorname{núcleo}^{*}\left(\operatorname{mask}\left(\widehat{L}, L_{0}\right)\right)$.

Dessa forma, corrige-se a cada $k \in \mathscr{H}$ e cada iteração $\eta$ do MV a estrutura do espaço $\mathbb{V}_{n \times n}^{\mathcal{N}}$ fazendo

$$
L^{k} \leftarrow \operatorname{mask}\left(L^{k}, \mathbf{L}_{0}^{k}\right)
$$

\subsection{Aproximação quadrática}

Para um certo instante de tempo $k \in \mathscr{H}$, dado um conjunto de ganhos $G_{p}$ em torno de uma estimativa $\widehat{G}$ e avaliados os custos de continuação a partir de $k$, isto é, $\left.J\right|_{k} ^{T-1}$ em função de $G_{p}$ para cada um deles, conforme dado na equação Equação 3.6, pode-se fazer um ajuste quadrático via mínimos quadrados para determinar as matrizes $A, b$ e $c$ de modo que

$$
f(G)=\operatorname{vec}(G)^{\prime} A \operatorname{vec}(G)+b^{\prime} \operatorname{vec}(G)+c,
$$

conforme escrito no Apêndice B.

Com isso, estima-se o sistema linear (matriz e termo independente) baseado nas observações dos pontos $G_{p}$ que é dada pela formulação do custo de continuação. Essa ideia foi cogitada porque algumas vezes as curvas de nível do custo (curvas de nível para uma quadrática) não eram muito suaves, possivelmente devido a erros de arredondamento. A ideia seria suavizar as curvas para achar seu mínimo. Ainda que válida, essa ideia não rendeu frutos, conforme será visto na seção de resultados mais adiante. 


\subsection{Metodologia de avaliação}

Os perfis de desempenho (ou em inglês Performance Profile, vide Gould e Scott (2016)) são uma ferramenta para comparar o desempenho de métodos ao se minimizar alguma estatística, como por exemplo, menor tempo de execução, menor número de iterações, menor valor de função objetivo, etc.

Trata-se de um gráfico que apresenta o percentual de "vitórias" de um determinado método sobre os demais. Um parâmetro de relaxamento/subotimalidade $f$ é também adicionado, de forma que: se $f=1$, então o perfil de desempenho é calculado em relação ao "valor ótimo"; se $f=2$, então o perfil contabiliza vitórias para todos os casos em que a estatística é menor ou igual ao dobro do ótimo; e assim por diante. De uma forma geral, quanto maior o valor do perfil de desempenho, melhor o método. Veja o exemplo abaixo.

Tabela 1 - Exemplo de Perfil de Desempenho.

\begin{tabular}{c|c|c|c|c|c||c||c}
\hline instâncias » & $\mathrm{a}$ & $\mathrm{b}$ & $\mathrm{c}$ & $\mathrm{d}$ & $\mathrm{e}$ & Perfil com $f=1$ & Perfil com $f=1.5$ \\
\hline Método A & 120 & 53 & 57 & 35 & 17 & $(1+0+0+1+0) / 5=40 \%$ & $(1+0+0+1+1) / 5=60 \%$ \\
Método B & 123 & 20 & 30 & 35 & 12 & $(0+1+1+1+1) / 5=80 \%$ & $(1+1+1+1+1) / 5=100 \%$ \\
\hline $1 \times$ "Ótimo" & 120 & 20 & 30 & 35 & 12 & - & - \\
\hline $1.5 \times$ "Ótimo" & 180 & 30 & 45 & 52.5 & 18 & - & - \\
\hline
\end{tabular}

Como pode-se ver na Tabela 1, os melhores valores obtidos em cada uma das instâncias foram 120, 20, 30, 35 e 12. Esses números são os "ótimos". Com isso, o perfil do método A para $f=1$ é o percentual de vezes que ele teve desempenho melhor (valor menor) ou igual a esses dados, sendo 2 vitórias e 3 derrotas, resultando em um perfil de $40 \%$ na "otimalidade" $(f=1)$. Por outro lado, se fizer um relaxamento, buscando por "subotimalidade", em que é aceito um valor até no máximo de 1.5 vezes o valor “ótimo", então o perfil do método A tem 3 vitórias e portanto, $60 \%$.

Como não há bancos de testes para este tipo de problema, serão feitos experimentos com instâncias geradas aleatoriamente, como também fizeram Bortolin (2012), Oliveira (2014) e Silva (2012), usando o gerador de SLSMs por eles implementado.

O problema abordado é o Regulador Linear Quadrático, com horizonte finito, sem observação do estado markoviano. As implementações são feitas em Matlab2017a, executadas em uma máquina Windows 8 com 64GB de memória RAM e processador Intel Core i7-4930K base x64 e CPU 3.4GHz.

O ótimo neste contexto se refere ao menor valor obtido, não o menor valor possível. 



\section{5}

\section{RESULTADOS E DISCUSSÕES}

Com as estratégias das máscaras e a aproximação quadrática, de precondicionamento e varredura abordados no Capítulo 4, foram realizados testes computacionais em 1000 instâncias geradas aleatoriamente, sendo resolvidas e comparados os seus custos "ótimos". Uma observação bastante importante é que o termo "ótimo" a ser empregado daqui em diante se refere ao menor valor obtido na execução da instância, dados os seus critérios de parada, e não ao menor valor possível. Dessa forma o termo indicará uma solução ótima ou subótima. O tamanho do horizonte foi sorteado $T \sim U[10,100]$, bem como o número máximo de iterações maxit $\sim$ $U[10,100]$. Os critérios de parada do algoritmo foram o número de iterações e o tempo máximo de cinco minutos.

As cadeias de Markov geradas têm de 2 a 5 estados, podendo ou não serem periódicas. Não foi dado atenção à periodicidade das cMs, fugindo do enfoque numérico. A dimensão de $x$ e $u$ para as instâncias geradas também se compreenderam entre 2 e 5 . Aplicações realísticas podem ter mais estados e dimensões maiores.

Primeiro serão discutidas as máscaras, a aproximação quadrática, os precondicionadores com e sem a varredura, todos com um método sorteado aleatoriamente dentre os oito propostos. Por fim, depois de discutidas as estatégias, compara-se os métodos utilizando as melhores delas, bem como os resultados obtidos. Outros gráficos tidos como menos importantes serão deixados no Apêndice D.

\subsection{Máscaras}

Nesta seção é apresentado o gráfico comparando os custos utilizando as máscaras versus sem elas.

Como se pode ver claramente no gráfico da Figura 19, o uso das máscaras na variável $L$ não melhorou os custos/convergência do MV significativamente. Isso se deve talvez ao fato de 
Figura 19 - Comparação dos custos com as máscaras e sem elas, para um total de 1000 instâncias.
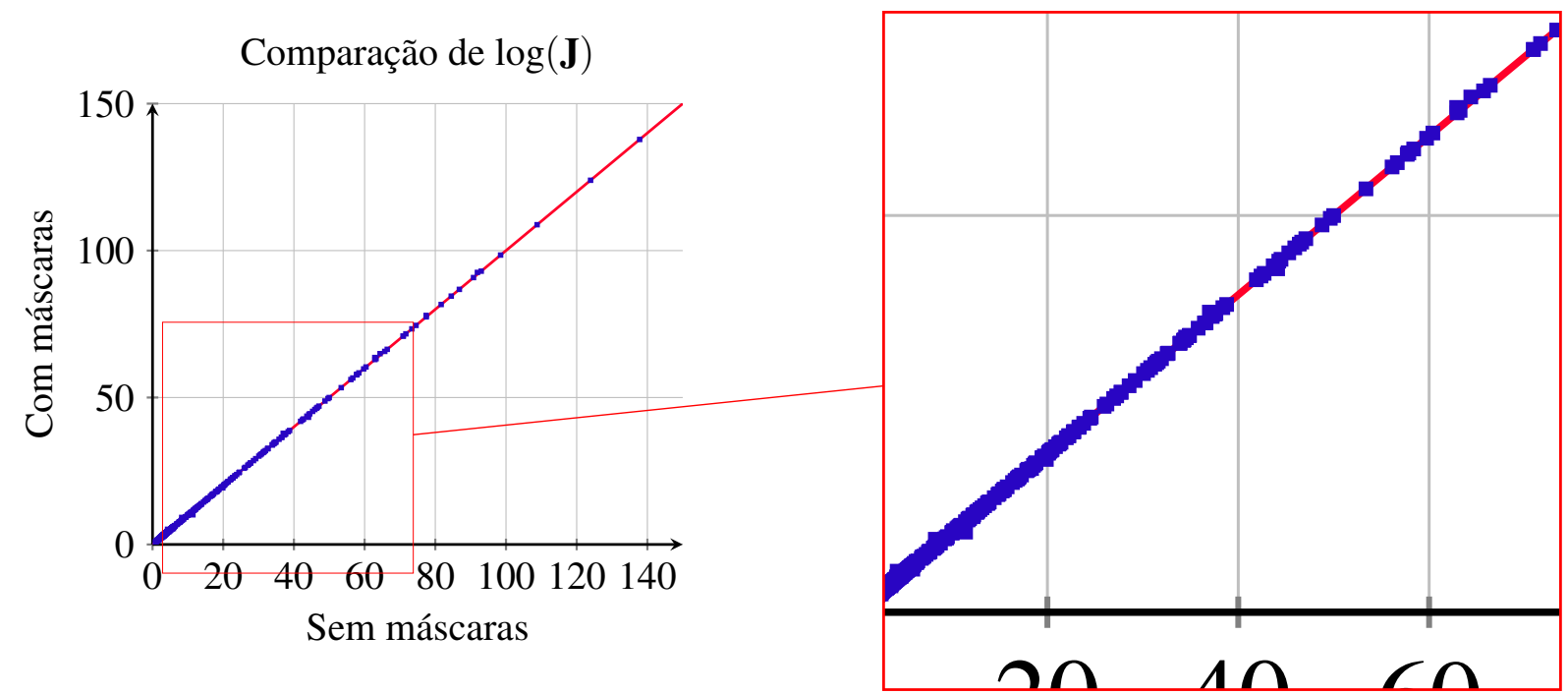

o horizonte escolhido ser pequeno, em comparação com aplicações mais realísticas, contexto em que elas podem ter um efeito mais pronunciado. Outro motivo talvez seja porque a grande maioria das matrizes $L$ tem posto completo nas instâncias testadas, como foi detectado em simulações, de forma que as máscaras são nada mais do que matrizes identidade e assim não têm nenhum efeito. Neste último caso, se $L$ tem posto baixo, então naturalmente as máscaras terão algum impacto, pois serão diferentes da matriz identidade. Outros testes utilizando as máscaras com os precondicionadores propostos não obtiveram nenhuma melhora.

\subsection{Aproximação quadrática multivariada}

Nesta seção é apresentado o gráfico comparando os custos utilizando a estratégia de aproximação quadrática para o cálculo dos ganhos.

Figura 20 - Comparação dos custos com aproximação quadrática e sem ela.

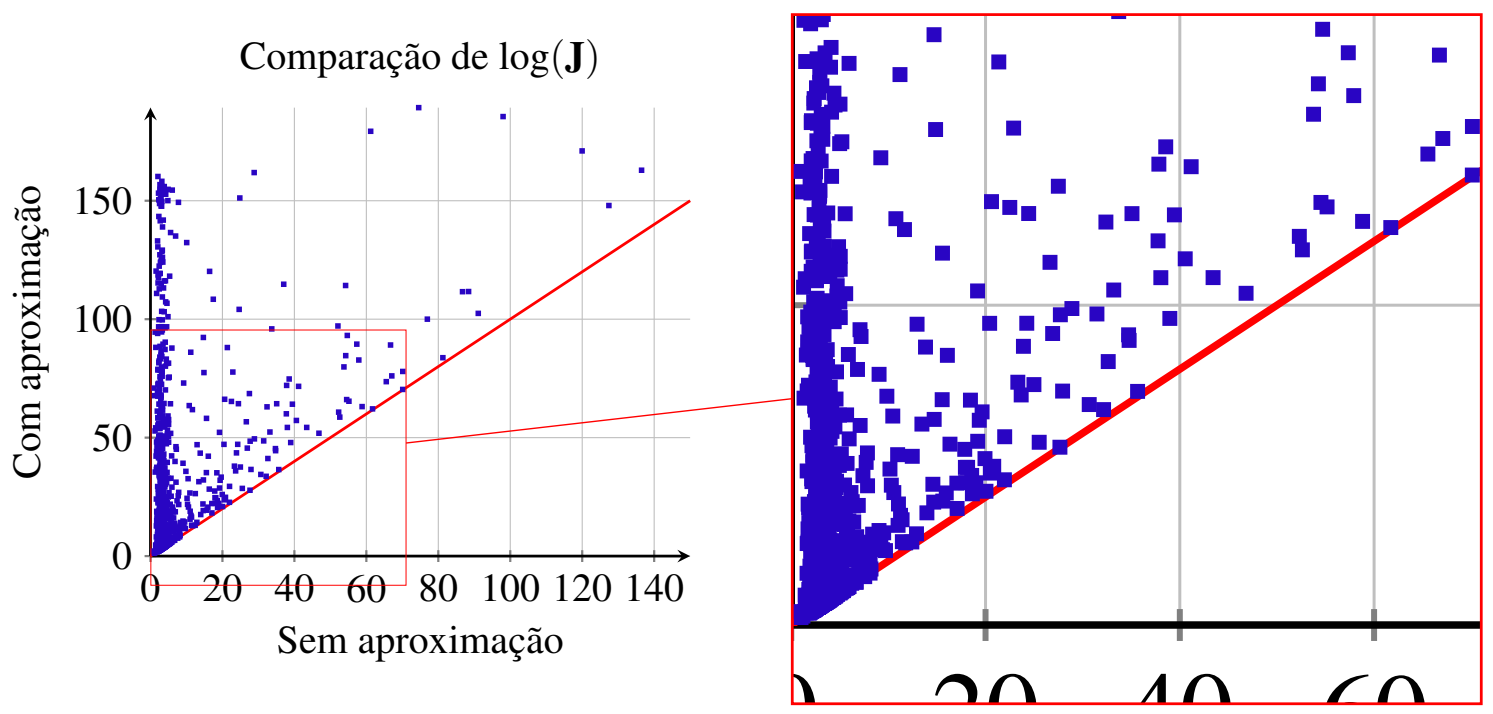


Como se pode ver claramente no gráfico da Figura 20, o uso da aproximação quadrática na busca do ganho ótimo $G$ piorou muito os custos. Talvez seja porque o domínio no qual os ganhos $G_{p}$ foram sorteados não foi adequado. Ainda assim, parece que essa abordagem não é muito frutífera.

\subsection{Precondicionadores}

O desempenho dos Precondicionadores 1,2,3,4,5, desde a Definição 8 até a Definição 12 serão comparados nesta seção. Para facilitar a leitura serão dadas etiquetas para os precondicionadores, conforme a Tabela 2.

\begin{tabular}{c|l} 
Etiqueta & Descrição \\
\hline P0 & Nenhum precondicionador adicionado; \\
P1 & Precondicionador da Definição 8; \\
P2 & Precondicionador da Definição 9; \\
P3 & Precondicionador da Definição 10; \\
P4 & Precondicionador da Definição 11; \\
P5 & Precondicionador da Definição 12; \\
\hline
\end{tabular}

Tabela 2 - Descrição das etiquetas dos precondicionadores utilizadas no texto.

Figura 21 - Comparação dos custos com uso do P1 e sem ele.

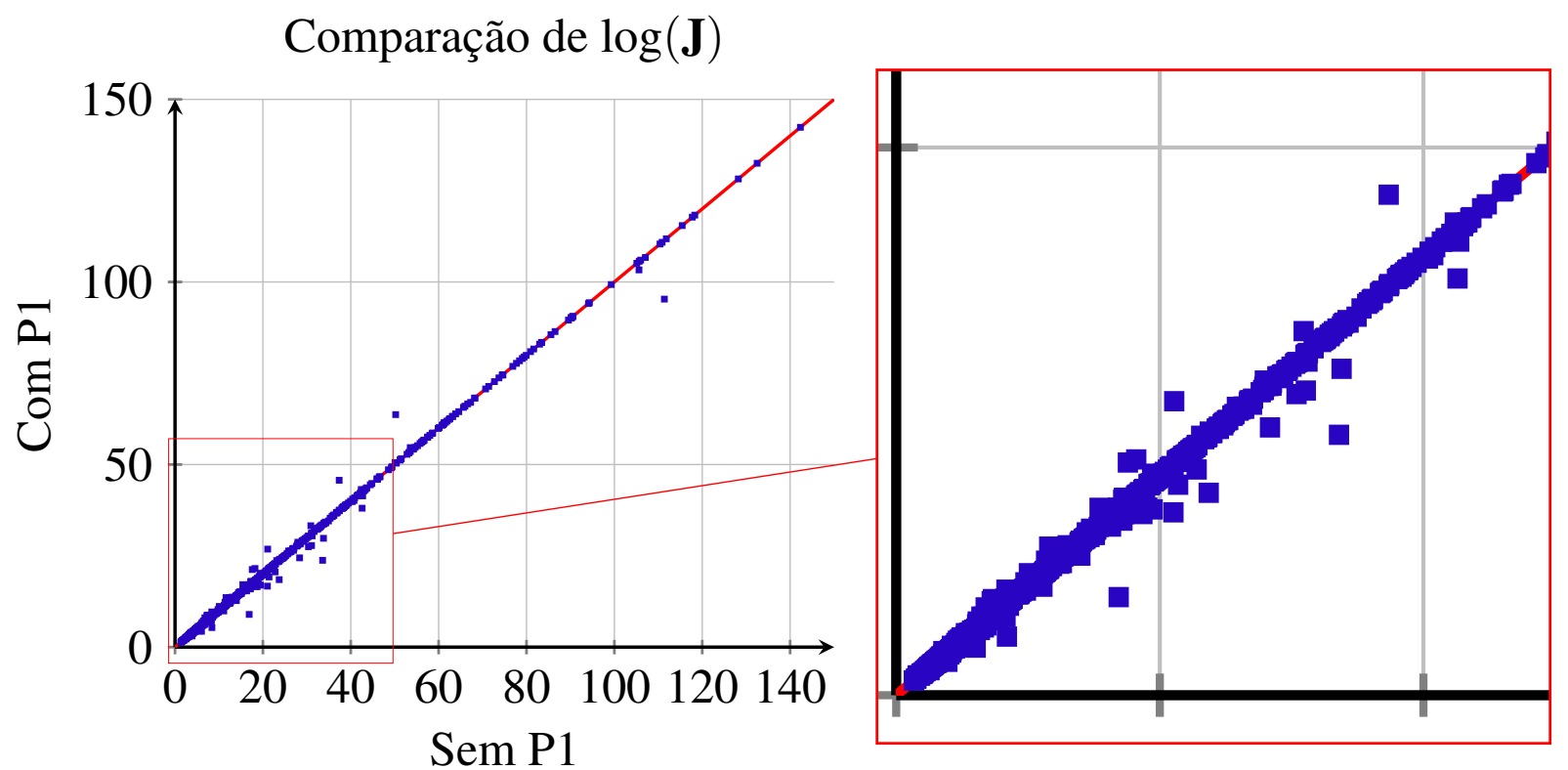

Como se pode ver claramente no gráfico da Figura 21, o uso do P1 no sistema linear $\mathbb{S}_{k}$ melhorou os custos de várias instâncias, mas também piorou o custo em várias outras, de forma que, no geral, não obteve melhora significativa. 
Figura 22 - Comparação dos custos com uso do P2 e sem ele.

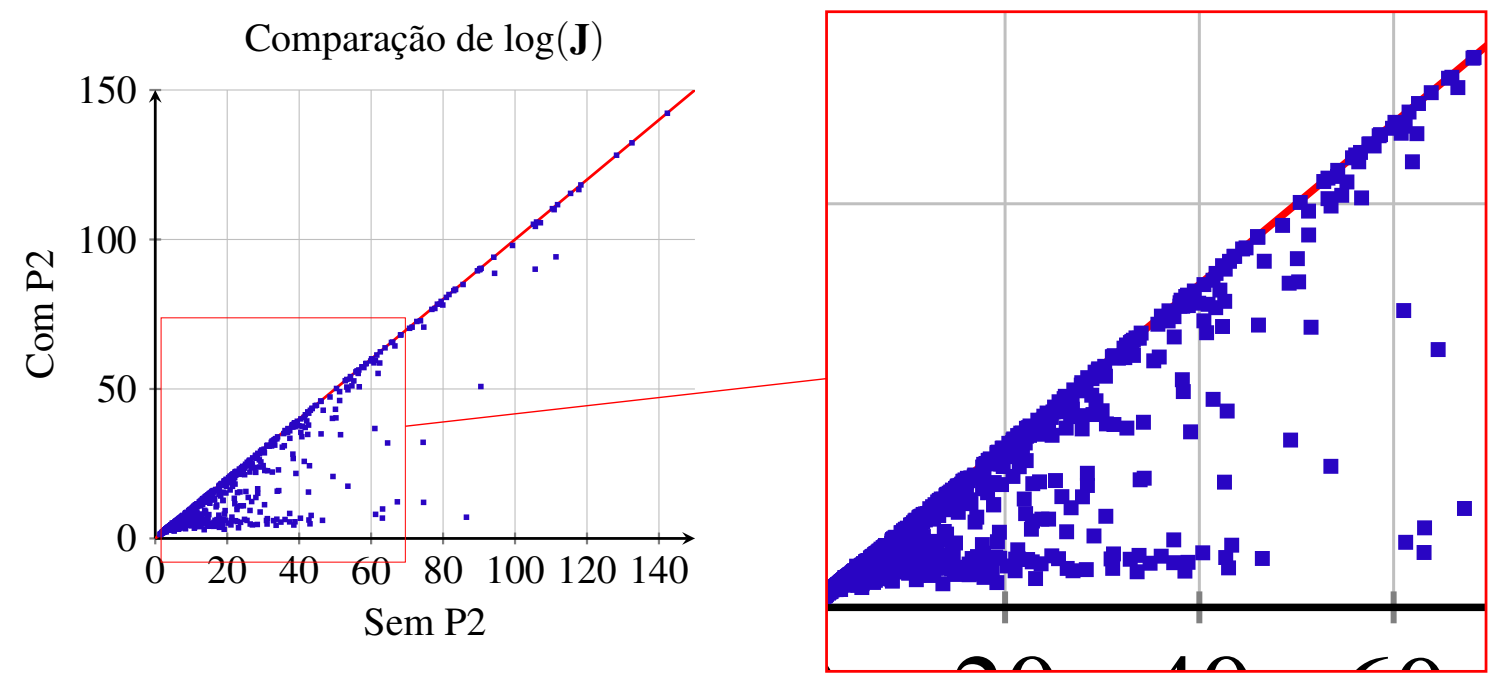

Como se pode ver claramente no gráfico da Figura 22, o uso do P2 no sistema linear $\mathbb{S}_{k}$ melhorou bastante os custos de várias instâncias, o que justifica os diferentes modelos para mudança de base criados: comparando-se P1 e P2, a única diferença entre eles é a ordem de precedência entre a soma e a multiplicação, que é sabido ser indiferente, mas que numericamente teve um efeito muito mais positivo quando se deu prioridade à multiplicação das matrizes para depois somá-las, em vez de somá-las e depois multiplicá-las. Detectado inicialmente este fenômeno, buscou-se outras formulações, no intuito de encontrar outras que fossem ainda melhores, o que resultou em P3 e P4. Para diversificar as abordagens, foi testada outra ideia, resultando no P5.

Os gráficos relativos aos $\mathrm{P} 3$ e P4 são semelhantes ao do P2, e por isso foram dispostos no Apêndice D.

Figura 23 - Comparação dos custos com uso do P5 e sem ele.

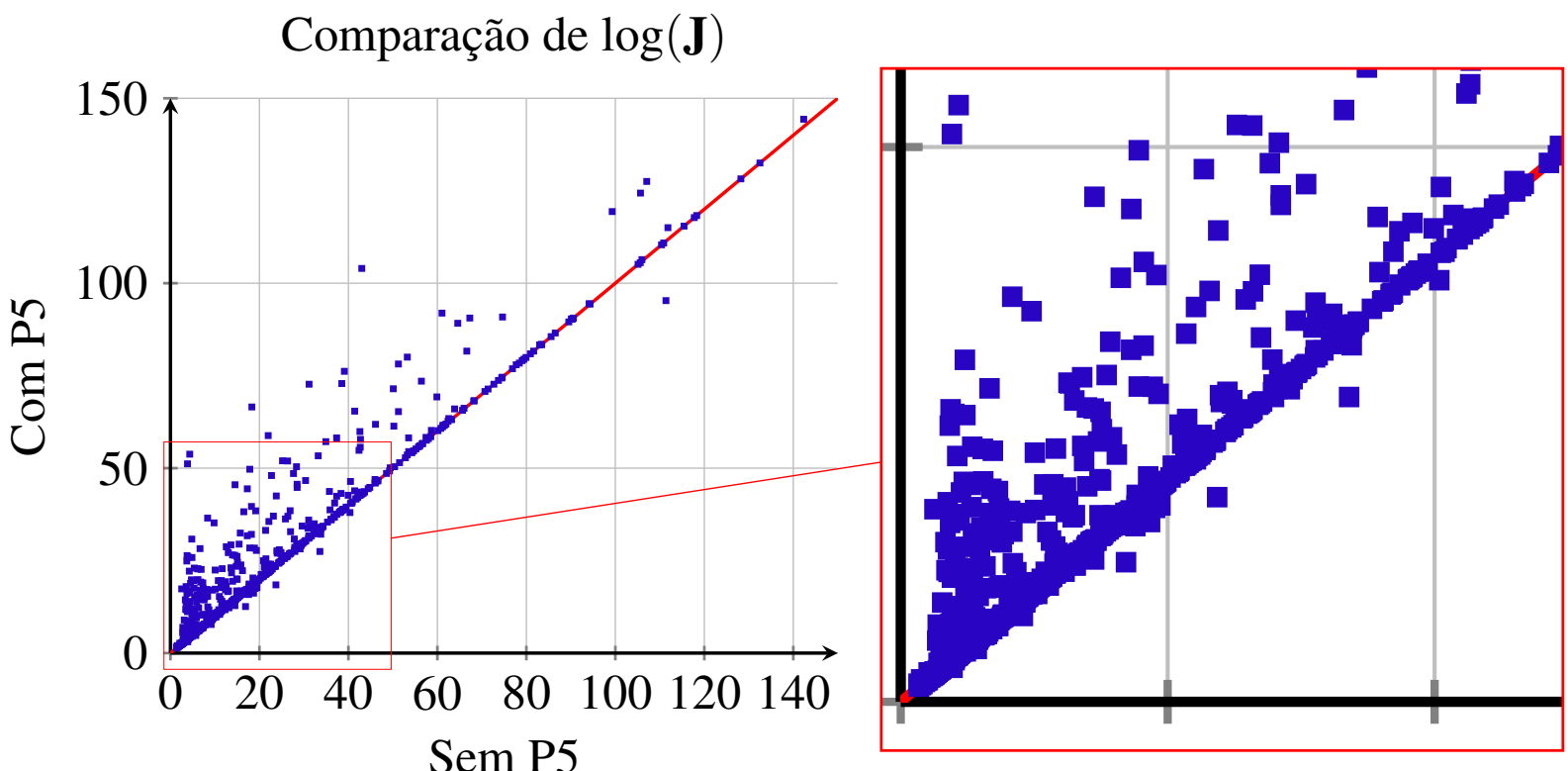


Claramente se observa que o P5 não é um bom candidato, pois piorou grandemente o desempenho do MV.

Figura 24 - Perfis de desempenho dos Precondicionadores.
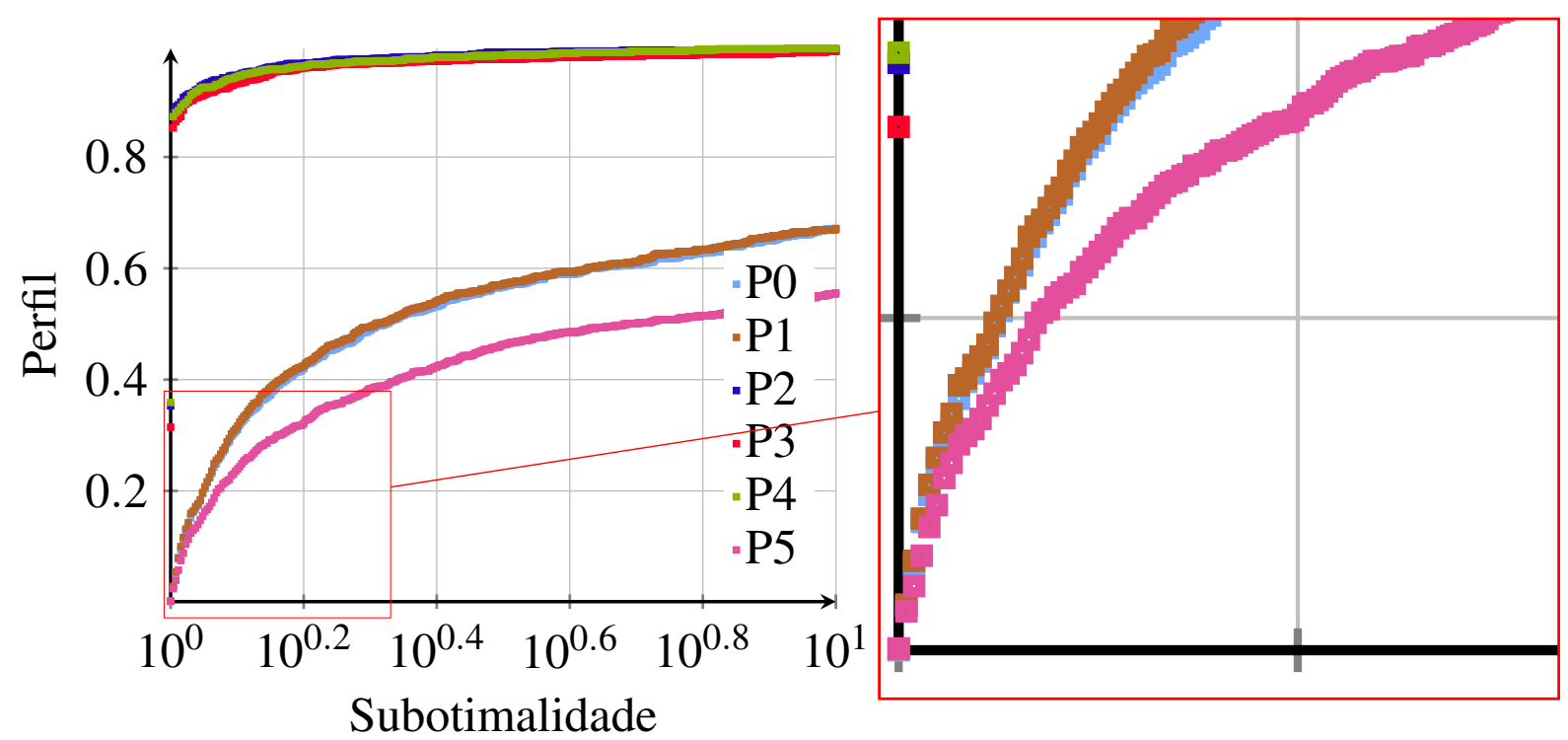

Pode-se observar no gráfico da Figura 24 que P2, P3 e P4 (gráficos em azul, vermelho e verde respectivamente) obtiveram os melhores desempenhos na otimalidade (representado por $10^{0}$ ) e na subotimalidade, até $10^{1}$ vezes o custo ótimo. O desempenho do P1 (em marrom) é ligeiramente melhor que aquele em que não foi usado nenhum precondicionador (em ciano), indicado por P0. O P5 (em magenta) obteve o pior desempenho.

\subsection{Varredura}

Buscando saber se o parâmetro $\alpha$ indicado nas definições dos precondicionadores melhora ou não o desempenho dos mesmos, foram realizados alguns testes. Como visto, $\alpha=2$ é o parâmetro que faz com que o número de condição do sistema linear seja 1. Aqui foram testados valores no conjunto $\{1+0.2 k, k \in\{0, \ldots, 10\}\}$ e escolhido aquele que deixou o menor resíduo.

Como visto na Figura 25, a estratégia de variar o parâmetro $\alpha$ com esses valores dados acima não melhoraram muito os resultados, tendo algum impacto positivo, mas não muito. Talvez outros valores para $\alpha$ deem melhores resultados. 
Figura 25 - Comparação do uso da estratégia de varredura dos valores de $\alpha$.

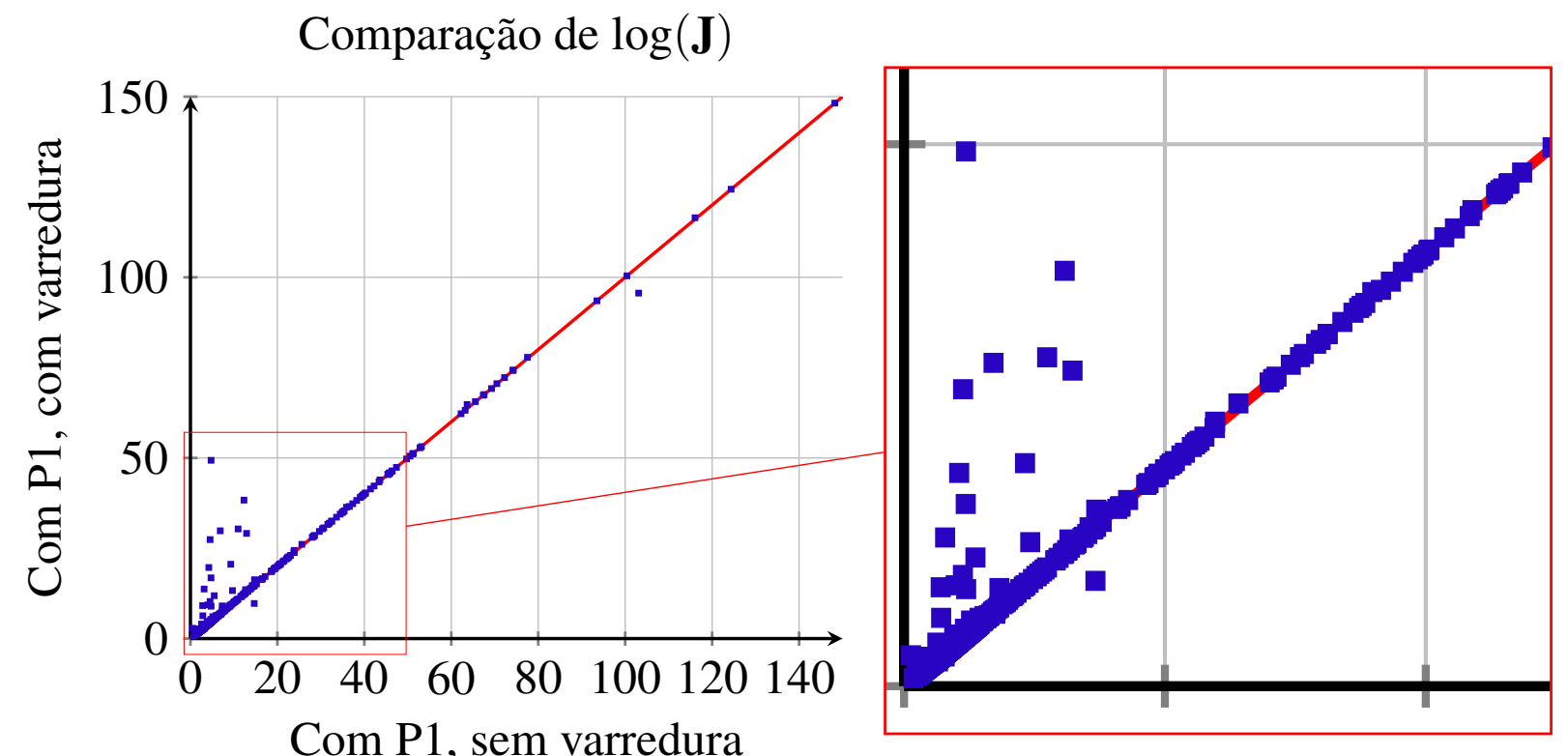

\subsection{Métodos}

Em se tratando dos métodos de resolução de sistemas lineares, foram testados oito, sendo cinco deles nativos do pacote Matlab e outros três implementados. Eles serão referenciados pelas etiquetas M1 até M8, conforme a descrição a seguir.

\begin{tabular}{c|l} 
Etiqueta & Descrição \\
\hline M1 & Fatoração do tipo LDL (resolução de sistemas triangulares) - Matlab; \\
M2 & Pseudoinversa - Matlab; \\
M3 & Gradientes conjugados - implementado; \\
M4 & Fatoração do tipo LU (resolução de sistemas triangulares) - Matlab; \\
M5 & Gradientes biconjugados - Matlab; \\
M6 & Direções conjugadas - implementado; \\
M7 & Jacobi-Richardson com rotações de Givens - implementado; \\
M8 & Inversa - Matlab; \\
\hline
\end{tabular}

Tabela 3 - Descrição das etiquetas dos métodos utilizadas no texto.

Nota - O M7 usa rotações de Givens apenas para que sua convergência seja garantida. 
Figura 26 - Perfis de desempenho dos métodos de solução de sistemas lineares com P2.

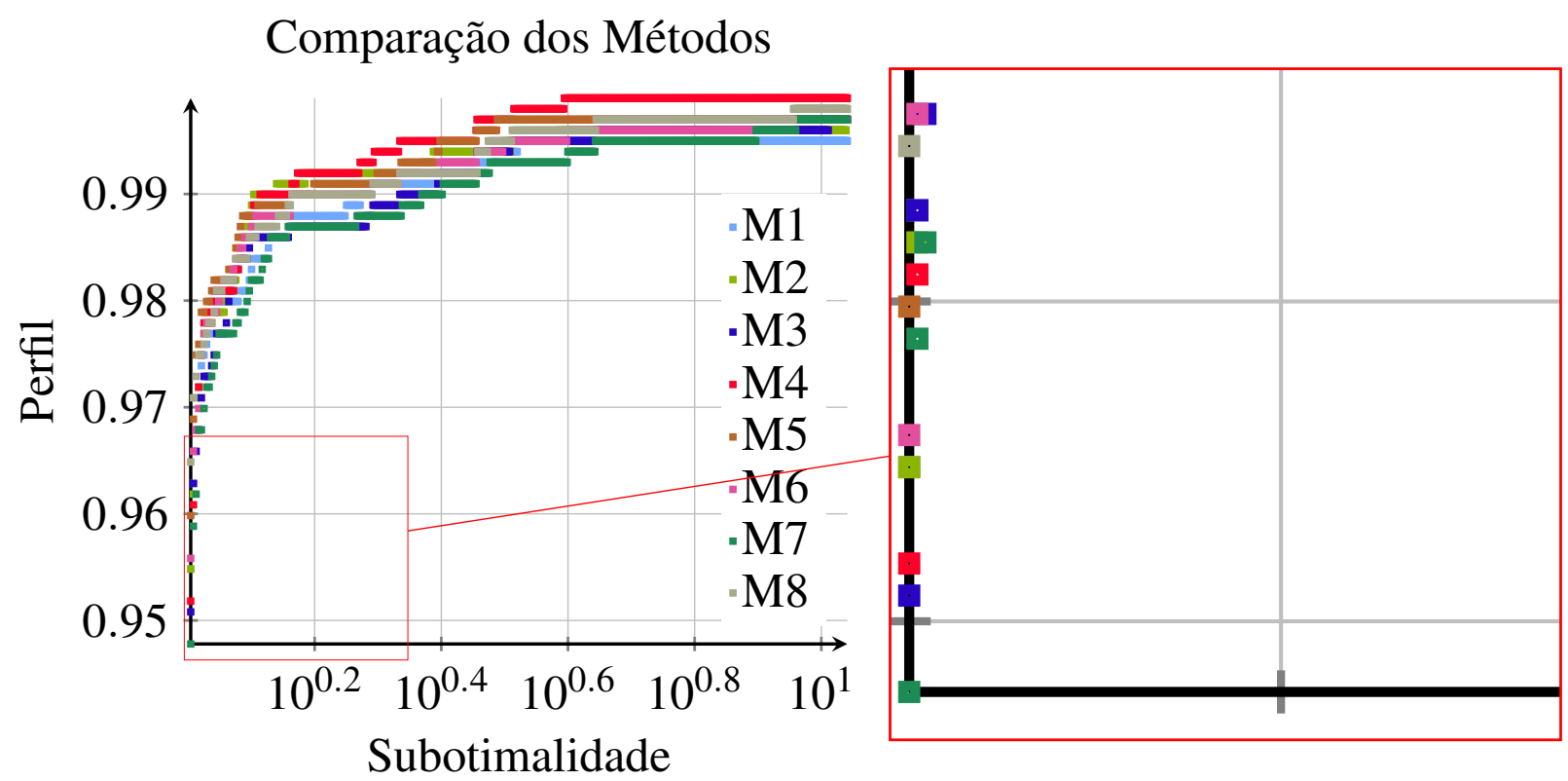

\begin{tabular}{rl|c|c|c|c|c|c|c} 
& M1 & M2 & M3 & M4 & M5 & M6 & M7 & M8 \\
\hline Desempenho & $15.96 \%$ & $14.55 \%$ & $16.56 \%$ & $13.15 \%$ & $17.46 \%$ & $15.16 \%$ & $11.34 \%$ & $17.47 \%$ \\
Tabela 4 - Comparação dos métodos na otimalidade (em $10^{\circ}$ ), com P2.
\end{tabular}

Observa-se nesses resultados que, de uma forma geral, todos os métodos obtiveram um desempenho semelhante, em torno de $11 \%$ a $17 \%$ na otimalidade. Particularmente, os métodos M4 e M7 não tiveram resultados tão bons quanto os demais na otimalidade, com o P2, ao passo que M3, M5 e M8 tiveram os melhores resultados, segundo a Tabela 4. Os M4 (vermelho), M5 (marrom) e M8 (cinza) foram os mais robustos na subotimalidade, como indicado na Figura 26. 
Figura 27 - Perfis de desempenho dos métodos de solução de sistemas lineares com P3.

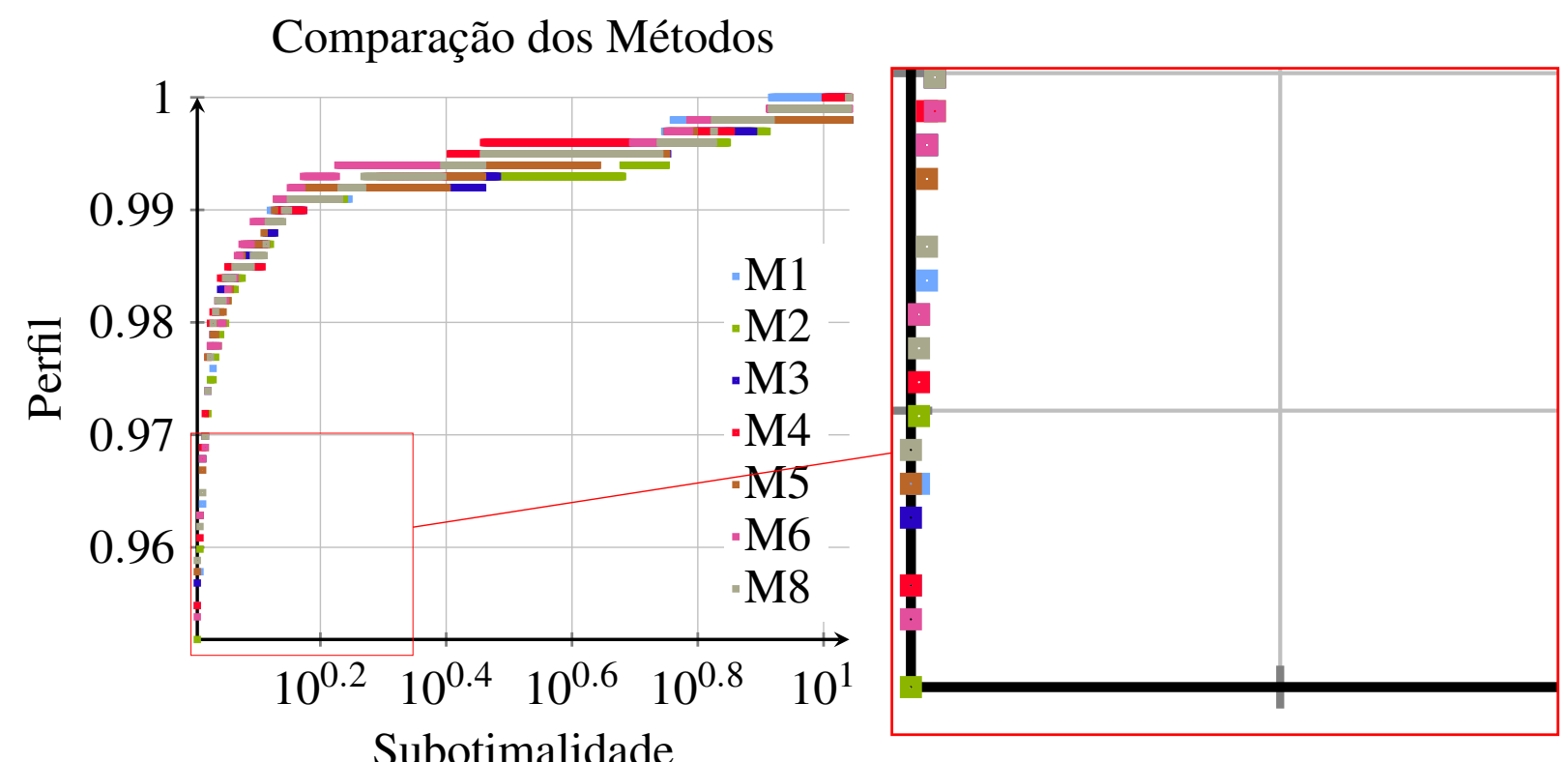

\begin{tabular}{c|c|c|c|c|c|c|c|c} 
& M1 & M2 & M3 & M4 & M5 & M6 & M7 & M8 \\
\hline Desempenho & $18.17 \%$ & $16.96 \%$ & $17.67 \%$ & $15.06 \%$ & $16.66 \%$ & $14.65 \%$ & $02.51 \%$ & $18.47 \%$
\end{tabular}

Tabela 5 - Comparação dos métodos na otimalidade $\left(\mathrm{em} 10^{\circ}\right)$, com P3.

Com o P3 o M7 teve um desempenho muito ruim, de forma que foi excluído do gráfico por dificultar a visualização dos demais. Observa-se nesses resultados que, de uma forma geral, todos os métodos obtiveram um desempenho semelhante, em torno de $14 \%$ a $18 \%$ na otimalidade. Particularmente, os métodos M4, M6 e M7 não tiveram resultados tão bons quanto os demais na otimalidade, com o P3, enquanto que M1, M3 e M8 tiveram os melhores resultados, segundo a Tabela 5. Os M4 (vermelho), M6 (magenta) e M8 (cinza) foram os mais robustos na subotimalidade, conforme visto na Figura 27. 
Figura 28 - Perfis de desempenho dos métodos de solução de sistemas lineares com P4.

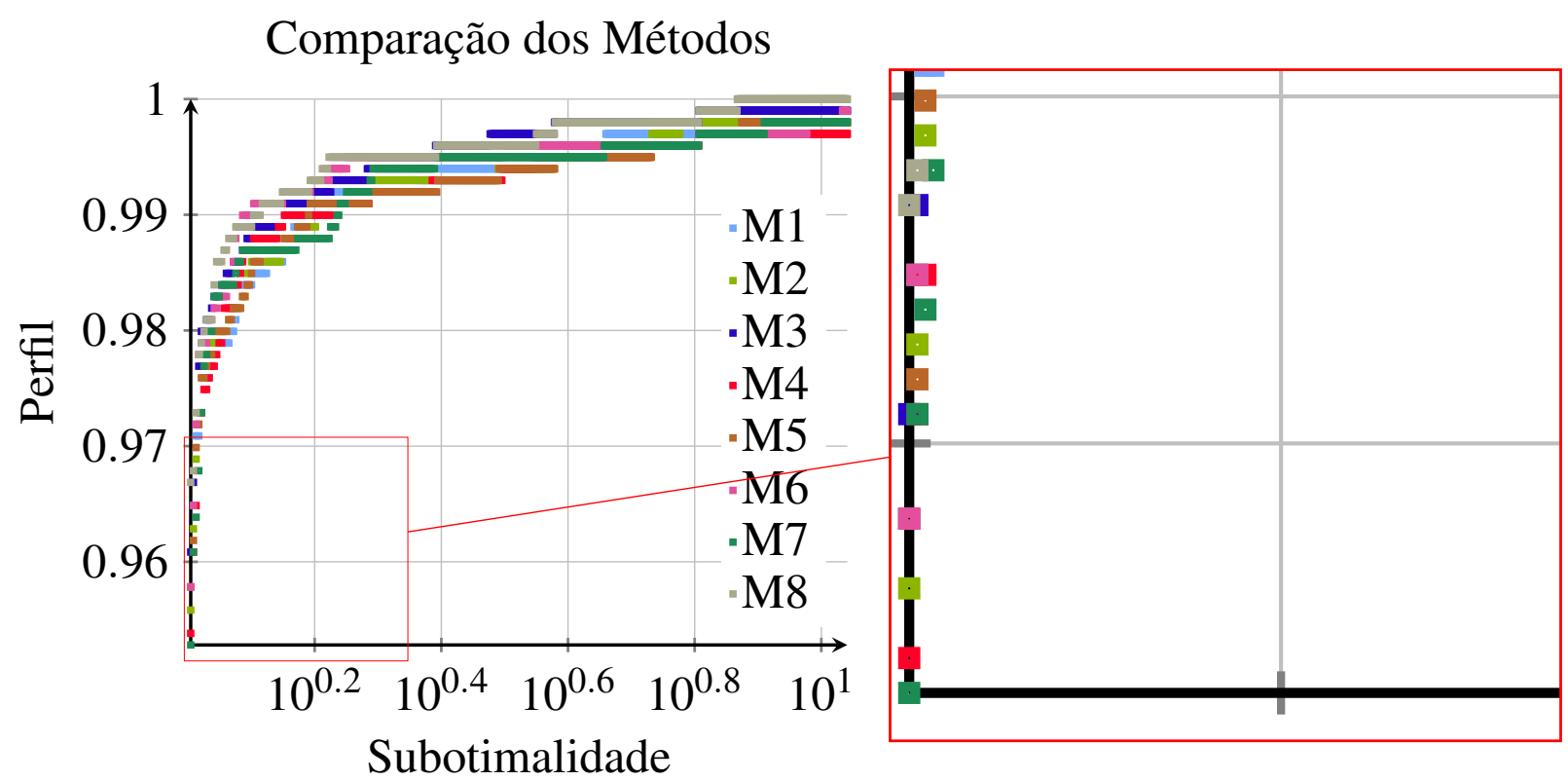

\begin{tabular}{rl|c|c|c|c|c|c|c|c} 
& M1 & M2 & M3 & M4 & M5 & M6 & M7 & M8 \\
\hline Desempenho & $14.65 \%$ & $13.75 \%$ & $17.77 \%$ & $14.65 \%$ & $15.56 \%$ & $15.06 \%$ & $13.15 \%$ & $16.76 \%$ \\
Tabela 6 - Comparação dos métodos na otimalidade (em $10^{\circ}$ ), com P4.
\end{tabular}

Observa-se nesses resultados que, de uma forma geral, todos os métodos obtiveram um desempenho semelhante, em torno de $13 \%$ a $17 \%$ na otimalidade. Particularmente, os métodos M2 e M7 não tiveram resultados tão bons quanto os demais na otimalidade, com o P4, enquanto que M3 e M8 foram os melhores, segundo a Tabela 6. Os M3 (azul), M6 (magenta) e M8 (cinza) foram os mais robustos na subotimalidade, conforme visto na Figura 28. 



\section{CONSIDERAÇÕES FINAIS}

O problema de controle do Regulador Linear Quadrático foi estudado com um horizonte finito em um sistema dinâmico discreto linear sujeito a saltos markovianos não observados em seus parâmetros, com um ruído aditivo.

O Método Variacional que vinha tendo bons índices de acordo com a literatura foi implementado e procuradas suas fragilidades. Percebeu-se que o método tinha uma convergência débil em alguns casos, provocada por erros numéricos em subproblemas mal condicionados. Detectada essa fraqueza, buscou-se formular técnicas de precondicionamento para seus subproblemas, resultando em quatro diferentes formulações baseadas na Decomposição em Valores Singulares (SVD) e outra baseada no Escalonamento de Jacobi.

Apesar de os quatro precondicionadores serem equivalentes matematicamente, foi percebida uma enorme diferença de performance quando se inverteu a ordem entre as operações de soma e multiplicação, sendo que os rotulados P2, P3 e P4 se saíram muito melhores diante dos P0 (sem precondicionador) e P1. O P5 (Escalonamento de Jacobi) foi o que obteve o pior desempenho, de modo que foi descartado dos testes finais, bem como P0 e P1.

Como um fenômeno tão curioso foi observado com relação aos erros numéricos, uma abordagem que consistiu de variar um certo expoente para cálculo da base para os precondicionadores foi testada, com pouco sucesso, de forma que o parâmetro $\alpha=2$ foi de fato o melhor entre eles, como indicado matematicamente.

Outra abordagem consistiu em tomar os parâmetros do modelo e aproximá-los por um modelo quadrático em mínimos quadrados, na busca de encontrar soluções melhores. No entanto, essa abordagem também não rendeu frutos.

Buscando evitar a perda da estrutura dos espaços nulos do parâmetro $L$ foram utilizadas máscaras de forma a reduzir os erros numéricos, conforme discutido na seção de implementação das mesmas. Essa abordagem não trouxe resultados significantes, talvez por causa das instâncias 
testadas.

Oito métodos de resolução de sistemas lineares do tipo $A x=b$ foram testados utilizando P2, P3 e P4, sendo que, de uma forma geral os métodos tiveram desempenho semelhante. Particularmente na otimalidade os métodos que tiveram melhor desempenho foram M3, M5 e M8 com P2; M1, M3 e M8 com P3; e M3 e M8 com P4. Na subotimalidade, os métodos que tiveram melhor desempenho foram M4, M5 e M8 com P2; M4, M6 e M8 com P3; e M3, M6 e M8 com P4.

\section{Sugestões de trabalhos futuros}

- Testar outros precondicionadores além do SVD e do Jacobi;

- Testar valores diferentes para $\alpha$ constante das Definição 8-Definição 12, buscando determinar o melhor parâmetro, se $\alpha=2$ ou se outro;

- Testar uma forma melhor de sortear os pontos $G_{p}$ na aproximação quadrática;

- Analisar outros modelos sem observação do estado contínuo, com e sem ruído;

- Ampliar o estudo para o problema de rastreamento;

- Testar outras amostras com propriedades variadas da teoria de controle, como observabilidade, detetabilidade, estabilizabilidade, dentre outras, utilizando-se das abordagens desenvolvidas. 
ABDOLlaHI, F. An H-infinity Dynamic Routing Control of Networked Multi-agent Systems. Tese (Doutorado), Montreal, P.Q., Canada, 2008. AAINR45644. Citado na página 26.

ABDOLLAHI, F.; KHORASANI, K. A decentralized markovian jump $h_{\infty}$ control routing strategy for mobile multi-agent networked systems. IEEE Transactions on Control Systems Technology, v. 19, n. 2, p. 269-283, 2011. Disponível em: <https://doi.org/10.1109/TCST.2010. 2046418>. Citado na página 26.

ABOU-KANDIL, H.; FREILING, G.; IONESCU, V.; JANK, G. Matrix Riccati Equations in Control and Systems Theory. Birkhauser Basel, 2003. Disponível em: <https://doi.org/10. 1007/978-3-0348-8081-7>. Citado na página 26.

ABOU-KANDIL, H.; FREILING, G.; JANK, G. On the solution of discrete-time markovian jump linear quadratic control problems. Automatica, v. 31, n. 5, p. $765-768$, 1995. ISSN 00051098. Disponível em: <http://www.sciencedirect.com/science/article/pii/000510989400164E>. Citado na página 26.

BORTOLIN, D. C. Métodos numéricos para o controle linear quadrático com saltos e observação parcial de estado. Dissertação (Mestrado) - Universidade de São Paulo, São Carlos, 2012. Disponível em: <https://doi.org/10.11606/D.55.2012.tde-28032012-151127>. Citado nas páginas 26, 35, 44 e 63.

CAJUEIRO, D. O. Stochastic optimal control of jumping Markov parameter processes with applications to finance. Tese (Doutorado) - Instituto Tecnológico de Aeronáutica-ITA, Brazil, 2002. Disponível em: <http://www.bd.bibl.ita.br/tesesdigitais/000490363.pdf>. Citado na página 26.

CUNHA, M. C. C. Método Numéricos. Campinas, SP: Editora Unicamp, 2000. Citado na página 51.

DYNKIN, E. B. Markov processes. Academic Press, v. 150, n. 3696, p. 1099-1131, 1965. Disponível em: <https://doi.org/10.1126/science.150.3696.598>. Citado nas páginas 25 e 34.

EUROPE, S. R. NAO Robot. 2017. Licensa (C) CC BY-SA 4.0. Disponível em: <https: //commons.wikimedia.org/wiki/File:NAO_Robot_.jpg>. Acesso em: 01/11/2018. Citado na página 27.

FRANCO, N. M. B. Cálculo Numérico. São Paulo, SP: Pearson Prentice Hall, 2013. Citado na página 51.

GONZÁLEZ, B. N. Palacio de la Bolsa de Madrid. 2017. Licensa (C) CC BY-SA 4.0. Disponível em: <https://commons.wikimedia.org/wiki/File:Valores,_Palacio_de_la_Bolsa,_Madrid, _Espa\%C3\%B1a,_2017.gif>. Acesso em: 01/11/2018. Citado na página 27. 
GOULD, N.; SCOTT, J. A note on performance profiles for benchmarking software. ACM Transactions on Mathematical Software, v. 42, n. 2, p. 15:1-15:5, 2016. Disponível em: $<$ http://dx.doi.org/10.1145/2950048>. Citado na página 62.

NASA. NASA Goddard Space Flight Center. 2016. Licensa (c) CC BY 2.0. Disponível em: <https://commons.wikimedia.org/wiki/File:Antares_Test_Flight_Scheduled_for_April_17_ (8632520692).jpg>. Acesso em: 01/11/2018. Citado na página 27.

NOCEDAL, J.; WRIGHT, S. J. Numerical optimization. 2. ed. New York, NY: Springer, 2006. Citado nas páginas 25, 51, 52 e 59 .

OLIVEIRA, L. T. de. Método variacional com atualização múltipla de ganhos para controle de sistemas lineares com parâmetros sujeitos a saltos Markovianos não observados. Dissertação (Mestrado) - Universidade de São Paulo, São Carlos, 2014. Disponível em: $<$ https://doi.org/10.11606/D.55.2014.tde-24092014-145046>. Citado nas páginas 26, 35, 44 e 63.

PETERSEN, P. Linear Algebra. New York: Springer, 2012. Disponível em: <https://doi.org/10. 1007/978-1-4614-3612-6>. Citado na página 59.

RIBEIRO, A. A.; KARAS, E. W. Otimização contínua: aspectos teóricos e computacionais. Curitiba, PR: Cengage Learning, 2014. Citado na página 25.

SILVA, C. A. Algoritmos para o custo médio a longo prazo de sistemas com saltos markovianos parcialmente observados. Tese (Doutorado) - Universidade de São Paulo, São Carlos, 2012. Disponível em: <https://doi.org/10.11606/T.55.2012.tde-25012013-095943>. Citado nas páginas 26, 35 e 63.

STOICA, A.; YAESH, I. Jump markovian-based control of wing deployment for an uncrewed air vehicle. Journal of Guidance, Control, and Dynamics, v. 25, n. 2, p. 407-411, 2002. Disponível em: <https://doi.org/10.2514/2.4896>. Citado na página 26.

TREFETHEN, L. N.; Bau III, D. Numerical Linear Algebra. Philadelphia, PA: SIAM, 1997. Citado nas páginas 52,53 e 58.

VAL, J. B. R. do; BAŞAR, T. Receding horizon control of jump linear systems and a macroeconomic policy problem. Journal of Economics Dynamics and Control, v. 23, n. 8, p. 1099-1131, 1999. Disponível em: <https://doi.org/10.1016/S0165-1889(98)00058-X>. Citado nas páginas 26,27 e 35.

VARGAS, A. do N. Controle por horizonte retrocedente de sistemas lineares com saltos markovianos e ruído aditivo. Dissertação (Mestrado) - Universidade Estadual de Campinas, Campinas, 2004. Disponível em: <http://repositorio.unicamp.br/jspui/handle/REPOSIP/260118>. Citado nas páginas 26 e 35.

VARGAS, A. N.; COSTA, E. F.; VAL, J. B. do. On the control of markov jump linear systems with no mode observation: application to a dc motor device. International Journal of Robust and Nonlinear Control, v. 23, n. 10, p. 1136-1150, 2013. Disponível em: <https://doi.org/10. 1002/rnc.2911>. Citado na página 26.

ZHOU, K.; DOYLE, J. C.; GLOVER, K. Robust and optimal control. Upper Saddle River, NJ: Prentice Hall, 1996. Citado nas páginas 46, 79 e 85. 
APÊNDICE

\section{A}

\section{CUSTO COMO UMA FUNÇÃO QUADRÁTICA EM $G_{k}$}

Primeiramente, apresentam-se as propriedades básicas dos operadores traço $\operatorname{tr}(\cdot)$ e vetorização vec $(\cdot)$, que podem ser vistas em Zhou, Doyle e Glover (1996).

$$
\begin{cases}\operatorname{tr}(A B C)=\operatorname{tr}(B C A)=\operatorname{tr}(C A B) & \text { (produto é cíclico) } \\ \|v\|^{2}=v^{\prime} v=\operatorname{tr}\left(v^{\prime} v\right)=\operatorname{tr}\left(v v^{\prime}\right) & \text { (linearidade) } \\ \operatorname{tr}(a B+C)=a \operatorname{tr}(B)+\operatorname{tr}(C) & \text { (vetorizado do traço de um produto) } \\ \operatorname{tr}(A B)=\operatorname{vec}\left(A^{\prime}\right)^{\prime} \operatorname{vec}(B)=\sum_{i} \sum_{j} a_{j i} b_{i j} & \text { (vetorizado do produto de 3 matrizes) } \\ \operatorname{vec}(A B C)=\left[C^{\prime} \otimes A\right] \operatorname{vec}(B) & \text { ind }\end{cases}
$$

em que $\otimes$ representa o produto de Kronecker.

A linearidade dos operadores traço $\operatorname{tr}(\cdot)$ e somatório $\sum(\cdot)$ serão amplamente utilizadas neste e nos demais apêndices, bem como a simetria de matrizes. Portanto o leitor deve estar atento a essas propriedades.

Relembrando alguns aspectos do problema, cujas definições serão copiadas aqui, vem:

$$
\left\{\begin{array} { l } 
{ \overline { \mathbf { A } } _ { i } ^ { k } = A _ { i } + B _ { i } G _ { k } } \\
{ \overline { \mathbf { C } } _ { i } ^ { k } = C _ { i } + G _ { k } ^ { \prime } D _ { i } G _ { k } } \\
{ \mathscr { E } ( U ) = \sum _ { j \in \mathscr { N } } \mathbb { P } _ { i j } U _ { j } } \\
{ X _ { i } ^ { k + 1 } = \mathscr { T } _ { \overline { \mathbf { A } } ^ { k } } ^ { i } ( X ^ { k } ) + \sum _ { i } ^ { k } } \\
{ \mathscr { T } _ { \overline { \mathbf { A } } } ^ { i } ( X ) = \sum _ { j \in \mathscr { N } } \mathbb { P } _ { j i } \overline { \mathbf { A } } _ { i } X _ { j } \overline { \mathbf { A } } _ { i } ^ { \prime } } \\
{ \sum _ { i } ^ { k } = \sum _ { j \in \mathscr { N } } \mathbb { P } _ { j i } \pi _ { k } ^ { j } H _ { j } H _ { j } ^ { \prime } }
\end{array} \quad \left\{\begin{array}{l}
\mathscr{L}_{\overline{\mathbf{A}}}^{i}(L)=\sum_{j \in \mathscr{N}} \mathbb{P}_{i j} \overline{\mathbf{A}}_{i}^{\prime} L_{j} \overline{\mathbf{A}}_{i} \\
L_{i}^{T}=0_{n \times n}, \quad \sigma_{i}^{T}=0 \quad \forall i \in \mathscr{N} \\
L_{i}^{k}=\overline{\mathbf{C}}_{i}^{k}+\mathscr{L}_{\overline{\mathbf{A}}^{k}}^{i}\left(L^{k+1}\right) \\
\sigma_{i}^{k}=\operatorname{tr}\left(\mathscr{E}^{i}\left(L^{k+1}\right) H_{i} H_{i}^{\prime}\right)+\mathscr{E}\left(\sigma^{k+1}\right) \\
\langle U, V\rangle=\sum_{i \in \mathscr{N}} \operatorname{tr}\left(U_{i}^{\prime} V_{i}\right) \\
i \in \mathscr{N}=\{1, \ldots, N\}, k \in \mathscr{H}=\{0, \ldots, T\}
\end{array}\right.\right.
$$

em que $X_{i}^{k}=\left(X_{i}^{k}\right)^{\prime}, C_{i}=C_{i}^{\prime}, \overline{\mathbf{C}}_{i}^{k}=\left(\overline{\mathbf{C}}_{i}^{k}\right)^{\prime}, \Sigma_{i}^{k}=\left(\Sigma_{i}^{k}\right)^{\prime}, D_{i}=D_{i}^{\prime}$ e $L_{i}^{k}=\left(L_{i}^{k}\right)^{\prime}$ são matrizes simétricas para todo $k \in \mathscr{H}$ e todo $i \in \mathscr{N}$. Também, o produto interno $\langle\cdot, \cdot\rangle$ definido em (A.2) é linear 
(verifique), propriedade que será utilizada a seguir. $\mathrm{O}$ custo total $\mathbf{J}$ é dado pelo somatório dos custos parciais $J_{k}$ :

$$
\begin{aligned}
\mathbf{J}=J_{0}+J_{1}+\ldots+J_{T-1} & =\left\langle X^{0}, C+G_{0}^{\prime} D G_{0}\right\rangle+\left\langle X^{1}, C+G_{1}^{\prime} D G_{1}\right\rangle+\ldots \\
\ldots+\left\langle X^{T-1}, C+G_{T-1}^{\prime} D G_{T-1}\right\rangle & =\sum_{k \in \mathscr{H} \backslash\{T\}}\left\langle X^{k}, \overline{\mathbf{C}}^{k}\right\rangle .
\end{aligned}
$$

Dados dois ganhos arbitrários $G_{T-2}$ e $G_{T-1}$ (com isso se tem $\overline{\mathbf{C}}^{T-1}$ e $\overline{\mathbf{C}}^{T-1}$ ) e somando os custos $J_{T-2}$ e $J_{T-1}$ do final do horizonte, tem-se:

$$
\begin{aligned}
J_{T-2}+J_{T-1} & =\left\langle X^{T-2}, \overline{\mathbf{C}}^{T-2}\right\rangle+\left\langle X^{T-1}, \overline{\mathbf{C}}^{T-1}\right\rangle \\
& =\left\langle X^{T-2}, \overline{\mathbf{C}}^{T-2}\right\rangle+\left\langle\mathscr{T}_{\overline{\mathbf{A}}^{T-2}}\left(X^{T-2}\right)+\Sigma^{T-2}, \overline{\mathbf{C}}^{T-1}\right\rangle \\
& =\left\langle X^{T-2}, \overline{\mathbf{C}}^{T-2}\right\rangle+\left\langle\mathscr{T}_{\overline{\mathbf{A}}^{T-2}}\left(X^{T-2}\right), \overline{\mathbf{C}}^{T-1}\right\rangle+\left\langle\Sigma^{T-2}, \overline{\mathbf{C}}^{T-1}\right\rangle \\
& =\left\langle X^{T-2}, \overline{\mathbf{C}}^{T-2}\right\rangle+\left\langle X^{T-2}, \mathscr{L}_{\overline{\mathbf{A}}^{T-2}}\left(\overline{\mathbf{C}}^{T-1}\right)\right\rangle+\left\langle\Sigma^{T-2}, \overline{\mathbf{C}}^{T-1}\right\rangle \\
& =\left\langle X^{T-2}, \overline{\mathbf{C}}^{T-2}+\mathscr{L}_{\overline{\mathbf{A}}^{T-2}}\left(\overline{\mathbf{C}}^{T-1}\right)\right\rangle+\left\langle\Sigma^{T-2}, \overline{\mathbf{C}}^{T-1}\right\rangle \\
& =\left\langle X^{T-2}, L^{T-2}\right\rangle+\underbrace{\left\langle\Sigma^{T-2}, \overline{\mathbf{C}}^{T-1}\right\rangle}_{\pi_{T-2}^{\prime} \sigma^{T-2}} \\
& =\left\langle X^{T-2}, L^{T-2}\right\rangle+\pi_{T-2}^{\prime} \sigma^{T-2} .
\end{aligned}
$$

$\mathrm{Na}$ terceira e quinta igualdades, a linearidade de $\langle\cdot, \cdot\rangle$ permite separar (agrupar) o termo em duas (uma) parcelas. Na quarta igualdade, o operador $\mathscr{L}_{(\cdot)}(\cdot)$ é o autoadjunto de $\mathscr{T}_{(\cdot)}(\cdot)$. Para provar isso, basta usar as definições de $\mathscr{L}, \mathscr{T}$ e $\langle\cdot, \cdot\rangle$, trocando-se a ordem dos somatórios e usando a propriedade 1 de (A.1), além das definições (A.2):

$$
\begin{aligned}
\left\langle\mathscr{T}_{\overline{\mathbf{A}}}(X), \overline{\mathbf{C}}\right\rangle & =\sum_{i \in \mathscr{N}} \operatorname{tr}\left(\mathscr{T}_{\overline{\mathbf{A}}}^{i}(X) \overline{\mathbf{C}}_{i}\right) \stackrel{(\mathrm{A} .2)}{=} \sum_{i \in \mathscr{N}} \operatorname{tr}\left(\left(\sum_{j \in \mathscr{N}} \mathbb{P}_{j i} \overline{\mathbf{A}}_{i} X_{j} \overline{\mathbf{A}}_{i}^{\prime}\right) \overline{\mathbf{C}}_{i}\right) \\
& \stackrel{(\mathrm{A} .1)}{=} \sum_{j \in \mathscr{N}} \sum_{i \in \mathscr{N}} \mathbb{P}_{j i} \mathbf{t r}\left(X_{j} \overline{\mathbf{A}}_{i}^{\prime} \overline{\mathbf{C}}_{i} \overline{\mathbf{A}}_{i}\right)=\sum_{j \in \mathscr{N}} \operatorname{tr}\left(X_{j} \sum_{i \in \mathscr{N}} \mathbb{P}_{j i} \overline{\mathbf{A}}_{i}^{\prime} \overline{\mathbf{C}}_{i} \overline{\mathbf{A}}_{i}\right) \\
& \stackrel{(\mathrm{A} .2)}{=} \sum_{j \in \mathscr{N}} \operatorname{tr}\left(X_{j} \mathscr{L}_{\overline{\mathbf{A}}}^{j}(\overline{\mathbf{C}})\right)=\left\langle X, \mathscr{L}_{\overline{\mathbf{A}}}(\overline{\mathbf{C}})\right\rangle .
\end{aligned}
$$

Para provar que $\left\langle\Sigma^{T-2}, \overline{\mathbf{C}}^{T-1}\right\rangle=\pi_{T-2}^{\prime} \sigma^{T-2}$, em que $\pi_{T-2}$ representa a distribuição de probabilidade de $\theta_{T-2}$, iniciam-se os parâmetros $L_{i}^{T}=0_{n \times n}, \sigma_{i}^{T}=0$ para todo $i \in \mathscr{N}$.

Por (A.2), tem-se que

$$
L_{i}^{T-1}=\overline{\mathbf{C}}_{i}^{T-1}, \quad \sigma_{i}^{T-1}=0, \quad \forall i \in \mathscr{N} .
$$

Como

$$
\Sigma_{i}^{T-2}=\sum_{j \in \mathscr{N}} \mathbb{P}_{j i} \pi_{T-2}^{j} H_{j} H_{j}^{\prime}, \quad \mathscr{E}^{j}\left(L^{T-1}\right)=\sum_{i \in \mathscr{N}} \mathbb{P}_{j i} \overline{\mathbf{C}}_{i}^{T-1}
$$


então

$$
\begin{aligned}
\left\langle\Sigma^{T-2}, L^{T-1}\right\rangle & =\left\langle\Sigma^{T-2}, \overline{\mathbf{C}}^{T-1}\right\rangle=\sum_{i \in \mathscr{N}} \operatorname{tr}\left(\Sigma_{i}^{T-2} \overline{\mathbf{C}}_{i}^{T-1}\right) \stackrel{(\mathrm{A} .1)}{=} \sum_{i \in \mathscr{N}} \operatorname{tr}\left(\overline{\mathbf{C}}_{i}^{T-1} \Sigma_{i}^{T-2}\right) \\
& =\sum_{i \in \mathscr{N}} \operatorname{tr}\left(\overline{\mathbf{C}}_{i}^{T-1} \sum_{j \in \mathscr{N}} \mathbb{P}_{j i} \pi_{T-2}^{j} H_{j} H_{j}^{\prime}\right)=\sum_{i \in \mathscr{N}} \sum_{j \in \mathscr{N}} \pi_{T-2}^{j} \operatorname{tr}\left(\mathbb{P}_{j i} \overline{\mathbf{C}}_{i}^{T-1} H_{j} H_{j}^{\prime}\right) \\
& =\sum_{j \in \mathscr{N}} \pi_{T-2}^{j} \operatorname{tr}\left(\sum_{i \in \mathscr{N}} \mathbb{P}_{j i} \overline{\mathbf{C}}_{i}^{T-1} H_{j} H_{j}^{\prime}\right)=\sum_{j \in \mathscr{N}} \pi_{T-2}^{j} \underbrace{\operatorname{tr}\left(\mathscr{E}^{j}\left(L^{T-1}\right) H_{j} H_{j}^{\prime}\right)}_{s_{j}^{T-2}} \\
& =\sum_{j \in \mathscr{N}} \pi_{T-2}^{j} s_{j}^{T-2}=\pi_{T-2}^{\prime} \sigma^{T-2},
\end{aligned}
$$

onde $\sigma^{T-2}=s^{T-2}$ nessa ocasião.

Fazendo algumas contas análogas, mas para $k \in\{T-3, T-2, T-1\}$, e dado um ganho arbitrário $G_{T-3}$, vem:

$$
\begin{aligned}
\sum_{k=T-3}^{T-1} J_{k} & =\left\langle X^{T-3}, \overline{\mathbf{C}}^{T-3}\right\rangle+\left\langle X^{T-2}, L^{T-2}\right\rangle+\pi_{T-2}^{\prime} \sigma^{T-2} \\
& =\left\langle X^{T-3}, \overline{\mathbf{C}}^{T-3}\right\rangle+\left\langle\mathscr{T}_{\overline{\mathbf{A}}^{T-3}}\left(X^{T-3}\right)+\Sigma^{T-3}, L^{T-2}\right\rangle+\pi_{T-2}^{\prime} \sigma^{T-2} \\
& =\left\langle X^{T-3}, \overline{\mathbf{C}}^{T-3}\right\rangle+\left\langle\mathscr{T}_{\overline{\mathbf{A}}^{T-3}}\left(X^{T-3}\right), L^{T-2}\right\rangle+\left\langle\Sigma^{T-3}, L^{T-2}\right\rangle+\pi_{T-2}^{\prime} \sigma^{T-2} \\
& =\left\langle X^{T-3}, \overline{\mathbf{C}}^{T-3}\right\rangle+\left\langle X^{T-3}, \mathscr{L}_{\overline{\mathbf{A}}^{T-3}}\left(L^{T-2}\right)\right\rangle+\left\langle\Sigma^{T-3}, L^{T-2}\right\rangle+\pi_{T-2}^{\prime} \sigma^{T-2} \\
& =\left\langle X^{T-3}, \overline{\mathbf{C}}^{T-3}+\mathscr{L}_{\overline{\mathbf{A}}^{T-3}}\left(L^{T-2}\right)\right\rangle+\underbrace{\left\langle\Sigma^{T-3}, L^{T-2}\right\rangle+\pi_{T-2}^{\prime} \sigma^{T-2}}_{\pi_{T-3}^{\prime} \sigma^{T-3}} \\
& =\left\langle X^{T-3}, L^{T-3}\right\rangle+\pi_{T-3}^{\prime} \sigma^{T-3} .
\end{aligned}
$$

Para provar que $\left\langle\Sigma^{T-3}, L^{T-2}\right\rangle+\pi_{T-2}^{\prime} \sigma^{T-2}=\pi_{T-3}^{\prime} \sigma^{T-3}$, procede-se de forma análoga à anterior, mas com alguns ajustes. Primeiramente, verifique que

$$
\begin{gathered}
\pi_{T-2}^{i} \stackrel{(2.2)}{=} \sum_{j \in \mathscr{N}} \mathbb{P}_{j i} \pi_{T-3}^{j} . \\
\left\langle\Sigma^{T-3}, L^{T-2}\right\rangle= \\
\sum_{i \in \mathscr{N}} \operatorname{tr}\left(L_{i}^{T-2} \Sigma_{i}^{T-3}\right)=\sum_{i \in \mathscr{N}} \operatorname{tr}\left(L_{i}^{T-2} \sum_{j \in \mathscr{N}} \mathbb{P}_{j i} \pi_{T-3}^{j} H_{j} H_{j}^{\prime}\right) \\
=\sum_{i \in \mathscr{N}} \sum_{j \in \mathscr{N}} \pi_{T-3}^{j} \operatorname{tr}\left(\mathbb{P}_{j i} L_{i}^{T-2} H_{j} H_{j}^{\prime}\right)=\sum_{j \in \mathscr{N}} \pi_{T-3}^{j} \operatorname{tr}\left(\sum_{i \in \mathscr{N}} \mathbb{P}_{j i} L_{i}^{T-2} H_{j} H_{j}^{\prime}\right) \\
=\sum_{j \in \mathscr{N}} \pi_{T-3}^{j} \underbrace{\operatorname{tr}\left(\mathscr{E} j\left(L^{T-2}\right) H_{j} H_{j}^{\prime}\right)}_{s_{j}^{T-3}}=\sum_{j \in \mathscr{N}} \pi_{T-3}^{j} s_{j}^{T-3}
\end{gathered}
$$


Para terminar, vem:

$$
\begin{aligned}
\left\langle\Sigma^{T-3}, L^{T-2}\right\rangle & +\pi_{T-2}^{\prime} \sigma^{T-2}=\sum_{j \in \mathscr{N}} \pi_{T-3}^{j} s_{j}^{T-3}+\sum_{i \in \mathscr{N}} \pi_{T-2}^{i} \sigma_{i}^{T-2} \\
& \stackrel{(\text { A.4) }}{=} \sum_{j \in \mathscr{N}} \pi_{T-3}^{j} s_{j}^{T-3}+\sum_{i \in \mathscr{N}}\left(\sum_{j \in \mathscr{N}} \mathbb{P}_{j i} \pi_{T-3}^{j}\right) \sigma_{i}^{T-2} \\
& =\sum_{i \in \mathscr{N}} \sum_{j \in \mathscr{N}} \pi_{T-3}^{j} s_{j}^{T-3}+\mathbb{P}_{j i} \pi_{T-3}^{j} \sigma_{i}^{T-2} \\
& =\sum_{i \in \mathscr{N}} \sum_{j \in \mathscr{N}} \pi_{T-3}^{j}\left(s_{j}^{T-3}+\mathbb{P}_{j i} \sigma_{i}^{T-2}\right) \\
& =\sum_{j \in \mathscr{N}} \pi_{T-3}^{j}\left(s_{j}^{T-3}+\sum_{i \in \mathscr{N}} \mathbb{P}_{j i} \sigma_{i}^{T-2}\right) \\
& =\sum_{j \in \mathscr{N}} \pi_{T-3}^{j} \underbrace{\left(\operatorname{tr}\left(\mathscr{E} j\left(L^{T-2}\right) H_{j} H_{j}^{\prime}\right)+\mathscr{E}{ }^{j}\left(\sigma^{T-2}\right)\right)}_{\sigma_{j}^{T-3}} \\
& \stackrel{\text { (A.2) } \sum_{j \in \mathscr{N}} \pi_{T-3}^{j} \sigma_{j}^{T-3}=\pi_{T-3}^{\prime} \sigma^{T-3},}{ }
\end{aligned}
$$

o que conclui a demonstração.

$\mathrm{O}$ argumento indutivo utilizado acima demonstra para todo $\ell \in \mathscr{H} \backslash\{T\}$ a seguinte expressão:

$$
\left.J\right|_{\ell} ^{T-1}=\sum_{k=\ell}^{T-1} J_{k}=\left\langle X^{\ell}, L^{\ell}\right\rangle+\pi_{\ell}^{\prime} \sigma^{\ell},
$$

o que é chamado de custo de continuação a partir do estágio $\ell$, de forma que, se $\ell=0$, então

$$
\mathbf{J}=\left\langle X^{0}, L^{0}\right\rangle+\pi_{0}^{\prime} \sigma^{0}
$$

Para escrever o custo $\mathbf{J}$ em função de um determinado ganho $G_{k}$, mais algumas contas são necessárias. Tomando um índice $k=\ell \in \mathscr{H} \backslash\{T\}$, o custo total pode ser escrito como

$$
\mathbf{J}=\sum_{k=0}^{\ell-1}\left\langle X^{k}, \overline{\mathbf{C}}^{k}\right\rangle+\left.J\right|_{\ell} ^{T-1}=\sum_{k=0}^{\ell-1}\left\langle X^{k}, \overline{\mathbf{C}}^{k}\right\rangle+\left\langle X^{\ell}, L^{\ell}\right\rangle+\pi_{\ell}^{\prime} \sigma^{\ell}
$$

que, conforme a Equação A.2, fica

$$
\mathbf{J}=\underbrace{\sum_{k=0}^{\ell-1}\left\langle X^{k}, \overline{\mathbf{C}}^{k}\right\rangle}_{\phi}+\underbrace{\pi_{\ell}^{\prime} \sigma^{\ell}+\left\langle X^{\ell}, \overline{\mathbf{C}}^{\ell}+\mathscr{L}_{\overline{\mathbf{A}}^{\ell}}\left(L^{\ell+1}\right)\right\rangle}_{\psi} .
$$

Aqui, o custo indicado por $\phi$ contabiliza de $k=0$ até $k=\ell-1$. O custo indicado por $\psi$ contabiliza de $k=\ell$ até $k=T-1$ (custo de continuação a partir de $\ell$ ). E no entanto, apenas a segunda parcela de $\psi$ depende do ganho $G_{\ell}$, conforme (A.2): veja que $\overline{\mathbf{C}}^{k}$ e $\overline{\mathbf{A}}^{k}$ (e portanto $L^{k}$ ) dependem de $G_{k}, X^{k}$ depende de $G_{k-1}$ e $\sigma^{k}$ depende de $L^{k+1}$, que por sua vez depende de $G_{k+1}$. 
Dessa forma, os termos que não dependem de $G_{\ell}$ são escritos como uma constante $c_{1}$. Então, tem-se a seguinte expressão que será desenvolvida:

$$
\mathbf{J}=c_{1}+\left\langle X^{\ell}, \overline{\mathbf{C}}^{\ell}+\mathscr{L}_{\overline{\mathbf{A}}^{\ell}}\left(L^{\ell+1}\right)\right\rangle
$$

Para simplificar a notação, escreve-se $X^{\ell}=X, G_{\ell}=G$ e $L^{\ell+1}=L$. Agora, as contas serão abertas e alguns termos constantes em relação a $G$ serão separados. As equações em (A.2) serão utilizadas, bem como a linearidade de $\sum(\cdot)$ e de $\operatorname{tr}(\cdot)$.

$$
\begin{aligned}
\mathbf{J} & =c_{1}+\sum_{i \in \mathscr{N}} \operatorname{tr}\left[X_{i}\left(\left(C_{i}+G^{\prime} D_{i} G\right)+\sum_{j \in \mathscr{N}} \mathbb{P}_{i j}\left(A_{i}+B_{i} G\right)^{\prime} L_{j}\left(A_{i}+B_{i} G\right)\right)\right] \\
& =c_{1}+\sum_{i \in \mathscr{N}}\left[\operatorname{tr}\left(X_{i} C_{i}\right)+\operatorname{tr}\left(X_{i} G^{\prime} D_{i} G\right)+\sum_{j \in \mathscr{N}} \mathbb{P}_{i j} \operatorname{tr}\left(X_{i}\left(A_{i}+B_{i} G\right)^{\prime} L_{j}\left(A_{i}+B_{i} G\right)\right)\right] \\
& =c_{2}+\sum_{i \in \mathscr{N}}\left[\operatorname{tr}\left(X_{i} G^{\prime} D_{i} G\right)+\sum_{j \in \mathscr{N}} \mathbb{P}_{i j} \operatorname{tr}\left(X_{i}\left(A_{i}^{\prime} L_{j} A_{i}+A_{i}^{\prime} L_{j} B_{i} G+G^{\prime} B_{i}^{\prime} L_{j} A_{i}+G^{\prime} B_{i}^{\prime} L_{j} B_{i} G\right)\right)\right] \\
& =c+\sum_{i \in \mathscr{N}}\left[\operatorname{tr}\left(X_{i} G^{\prime} D_{i} G\right)+\sum_{j \in \mathscr{N}} \mathbb{P}_{i j} \operatorname{tr}\left(X_{i}\left(A_{i}^{\prime} L_{j} B_{i} G+G^{\prime} B_{i}^{\prime} L_{j} A_{i}+G^{\prime} B_{i}^{\prime} L_{j} B_{i} G\right)\right)\right] \\
& =c+\sum_{i \in \mathscr{N}}\left[\operatorname{tr}\left(X_{i}\left[G^{\prime} D_{i} G+\sum_{j \in \mathscr{N}} \mathbb{P}_{i j} G^{\prime} B_{i}^{\prime} L_{j} B_{i} G\right]\right)+\sum_{j \in \mathscr{N}} \mathbb{P}_{i j} \operatorname{tr}\left(X_{i}\left[A_{i}^{\prime} L_{j} B_{i} G+G^{\prime} B_{i}^{\prime} L_{j} A_{i}\right]\right)\right] \\
& =c+\sum_{i \in \mathscr{N}}\left[\operatorname{tr}\left(X_{i} G^{\prime}\left[D_{i}+\sum_{j \in \mathscr{N}} \mathbb{P}_{i j} B_{i}^{\prime} L_{j} B_{i}\right] G\right)+2 \operatorname{tr}\left(X_{i}\left[\sum_{j \in \mathscr{N}} \mathbb{P}_{i j} A_{i}^{\prime} L_{j} B_{i}\right] G\right)\right] \\
& =c+\sum_{i \in \mathscr{N}} \operatorname{tr}\left(X_{i} G^{\prime} Z_{i} G\right)+2 \operatorname{tr}\left(X_{i} M_{i} G\right),
\end{aligned}
$$

em que

$$
\begin{aligned}
& c_{2}=c_{1}+\sum_{i \in \mathscr{N}} \operatorname{tr}\left(X_{i} C_{i}\right), \quad c=c_{2}+\sum_{i \in \mathscr{N}} \sum_{j \in \mathscr{N}} \mathbb{P}_{i j} \operatorname{tr}\left(X_{i} A_{i}^{\prime} L_{j} A_{i}\right), \\
& Z_{i}=D_{i}+\sum_{j \in \mathscr{N}} \mathbb{P}_{i j} B_{i}^{\prime} L_{j} B_{i}, \quad M_{i}=\sum_{j \in \mathscr{N}} \mathbb{P}_{i j} A_{i}^{\prime} L_{j} B_{i}, \quad \forall i \in \mathscr{N}
\end{aligned}
$$

Com isso, obtém-se

$$
\mathbf{J}=f(G)=c+\sum_{i \in \mathscr{N}} \operatorname{tr}\left(X_{i} G^{\prime} Z_{i} G\right)+2 \operatorname{tr}\left(X_{i} M_{i} G\right) .
$$

Se for manipulado mais um pouco, usando as propriedades 4 e 5 em (A.1), considerando a simetria de $X$ e $Z$, vem:

$$
\begin{gathered}
\operatorname{tr}\left(X_{i} M_{i} G\right)=\operatorname{vec}\left(M_{i}^{\prime} X_{i}\right)^{\prime} \operatorname{vec}(G) \\
\operatorname{tr}\left(X_{i} G^{\prime} Z_{i} G\right)=\operatorname{vec}\left(Z_{i} G X_{i}\right)^{\prime} \operatorname{vec}(G)=\operatorname{vec}(G)^{\prime} \operatorname{vec}\left(Z_{i} G X_{i}\right)=\operatorname{vec}(G)^{\prime}\left[X_{i} \otimes Z_{i}\right] \operatorname{vec}(G) .
\end{gathered}
$$

Assim, pode-se ainda, escrever a Equação A.6 como

$$
\mathbf{J}=f(G)=c+\operatorname{vec}(G)^{\prime}\left[\sum_{i \in \mathscr{N}} X_{i} \otimes Z_{i}\right] \operatorname{vec}(G)+2 \operatorname{vec}\left(\sum_{i \in \mathscr{N}} M_{i}^{\prime} X_{i}\right)^{\prime} \operatorname{vec}(G)
$$


Dessa forma, pode-se ver que o custo $\mathbf{J}$ é quadrático em $\mathbf{v e c}(G)$.

A função custo $f(G)$ pode, portanto, ser calculada usando as matrizes de ganho $G$ como na Equação A.6 ou usando os vetorizados dessas matrizes, vec $(G)$, como na Equação A.7.

Derivando a Equação A.7 em relação a $\operatorname{vec}(G)$ e avaliando em zero, tem-se o seguinte sistema linear (condição necessária de otimalidade):

$$
\sum_{i \in \mathscr{N}}\left[X_{i} \otimes\left(D_{i}+B_{i}^{\prime} \Lambda_{i} B_{i}\right)\right] \operatorname{vec}(G)=-\sum_{i \in \mathscr{N}} \operatorname{vec}\left(B_{i}^{\prime} \Lambda_{i} A_{i} X_{i}\right)
$$

em que $\Lambda_{i}=\mathscr{E}^{i}(L)$ 
APÊNDICE

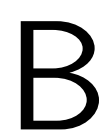

(2)

\section{APROXIMAÇÃO QUADRÁTICA MULTIVARIADA POR MÍNIMOS QUADRADOS}

Seja $x, b \in \mathbb{R}^{n}, A \in \mathbb{R}^{n \times n}$ e $c \in \mathbb{R}$ e considere a seguinte função quadrática $f: \mathbb{R}^{n} \rightarrow \mathbb{R}$ :

$$
f(x)=x^{\prime} A x+b^{\prime} x+c .
$$

Suponha que se tenha um conjunto de pontos $x_{p} \in \mathbb{R}^{n}$, com $p \in \mathscr{P}=\{1, \ldots, P\}$, e necessita-se calcular qual é a função quadrática que melhor se ajusta a eles. Para isso, precisa-se calibrar os parâmetros $A, b$ e $c$ de modo a representar os pontos o mais preciso possível. Esse problema pode ser escrito em termos de mínimos quadrados (minimizar o erro quadrático):

$$
\min _{A, b, c} M(A, b, c)=\sum_{p \in \mathscr{P}}\left[f\left(x_{p}\right)-\left(x_{p}^{\prime} A x_{p}+b^{\prime} x_{p}+c\right)\right]^{2} .
$$

De Zhou, Doyle e Glover (1996), a fórmula

$$
\frac{d \operatorname{tr}(P X Q)}{d X}=P^{\prime} Q^{\prime}
$$

pode ser empregada para calcular a seguinte expressão, que será usada para calcular a derivada de $M(A, b, c)$ em relação a $A$ na terceira equação do sistema abaixo (regra da cadeia):

$$
\frac{d\left(x^{\prime} A x\right)}{d A}=\frac{d \operatorname{tr}\left(x^{\prime} A x\right)}{d A}=x x^{\prime}
$$


Assim, calculando as derivadas e avaliando em zero, obtém-se o sistema:

$$
\left\{\begin{array}{l}
\left.\frac{\partial M(A, b, c)}{\partial c}\right|_{0}=2 \sum_{p \in \mathscr{P}}\left(f\left(x_{p}\right)-x_{p}^{\prime} A x_{p}-b^{\prime} x_{p}-c\right)(-1)=0 \\
\left.\frac{\partial M(A, b, c)}{\partial b}\right|_{0}=2 \sum_{p \in \mathscr{P}}\left(f\left(x_{p}\right)-x_{p}^{\prime} A x_{p}-x_{p}^{\prime} b-c\right)\left(-x_{p}\right)=0 \\
\left.\frac{\partial M(A, b, c)}{\partial A}\right|_{0}=2 \sum_{p \in \mathscr{P}}\left(f\left(x_{p}\right)-x_{p}^{\prime} A x_{p}-b^{\prime} x_{p}-c\right)\left(-x_{p} x_{p}^{\prime}\right)=0
\end{array}\right.
$$

Algumas das propriedades em (A.1) serão utilizadas:

$$
\begin{cases}\operatorname{tr}(A B C)=\operatorname{tr}(B C A)=\operatorname{tr}(C A B) & (i) \\ \operatorname{tr}(A B)=\operatorname{vec}\left(A^{\prime}\right)^{\prime} \operatorname{vec}(B) & (i i) \\ \operatorname{vec}(A B C)=\left[C^{\prime} \otimes A\right] \operatorname{vec}(B)\end{cases}
$$

O sistema passa a ser então:

$$
\left\{\begin{array}{l}
\sum_{p \in \mathscr{P}} x_{p}^{\prime} A x_{p}+b^{\prime} x_{p}+c=\sum_{p \in \mathscr{P}} f\left(x_{p}\right) \\
\sum_{p \in \mathscr{P}}\left(x_{p}^{\prime} A x_{p}\right) x_{p}+\left(b^{\prime} x_{p}\right) x_{p}+c x_{p}=\sum_{p \in \mathscr{P}} f\left(x_{p}\right) x_{p} \\
\sum_{p \in \mathscr{P}}\left(x_{p}^{\prime} A x_{p}\right) x_{p} x_{p}^{\prime}+\left(b^{\prime} x_{p}\right) x_{p} x_{p}^{\prime}+c x_{p} x_{p}^{\prime}=\sum_{p \in \mathscr{P}} f\left(x_{p}\right) x_{p} x_{p}^{\prime} .
\end{array}\right.
$$

Por $\left(x_{p}^{\prime} A x_{p}\right)$ ser escalar, na terceira equação, pode-se fazer $\left(x_{p}^{\prime} A x_{p}\right) x_{p} x_{p}^{\prime}=x_{p}\left(x_{p}^{\prime} A x_{p}\right) x_{p}^{\prime}$. $\mathrm{O}$ mesmo se aplica à parcela vizinha, $\left(b^{\prime} x_{p}\right) x_{p} x_{p}^{\prime}=x_{p}\left(b^{\prime} x_{p}\right) x_{p}^{\prime}$. E ainda, na segunda equação, pode-se fazer $\left(b^{\prime} x_{p}\right) x_{p}=x_{p}\left(b^{\prime} x_{p}\right)=x_{p}\left(x_{p}^{\prime} b\right)$ utilizando a mesma ideia, bem como inverter a ordem dos fatores nas parcelas vizinhas, o que dá:

$$
\left\{\begin{array}{l}
\sum_{p \in \mathscr{P}} c+b^{\prime} x_{p}+x_{p}^{\prime} A x_{p}=\sum_{p \in \mathscr{P}} f\left(x_{p}\right) \\
\sum_{p \in \mathscr{P}} x_{p} c+x_{p}\left(x_{p}^{\prime} b\right)+x_{p}\left(x_{p}^{\prime} A x_{p}\right)=\sum_{p \in \mathscr{P}} f\left(x_{p}\right) x_{p} \\
\sum_{p \in \mathscr{P}} x_{p} x_{p}^{\prime} c+x_{p}\left(b^{\prime} x_{p}\right) x_{p}^{\prime}+x_{p}\left(x_{p}^{\prime} A x_{p}\right) x_{p}^{\prime}=\sum_{p \in \mathscr{P}} f\left(x_{p}\right) x_{p} x_{p}^{\prime} .
\end{array}\right.
$$

Na primeira equação,

$$
x_{p}^{\prime} A x_{p}=\operatorname{tr}\left(x_{p}^{\prime} A x_{p}\right) \stackrel{(i)}{=} \operatorname{tr}\left(x_{p} x_{p}^{\prime} A\right) \stackrel{(i i)}{=} \operatorname{vec}\left(x_{p} x_{p}^{\prime}\right)^{\prime} \operatorname{vec}(A) .
$$

Na segunda equação, utilizando esta última expressão, fica

$$
x_{p}\left(x_{p}^{\prime} A x_{p}\right)=x_{p} \operatorname{vec}\left(x_{p} x_{p}^{\prime}\right)^{\prime} \operatorname{vec}(A) .
$$

Na terceira equação, vetorizando as duas últimas parcelas do primeiro membro, vem

$$
\operatorname{vec}\left(x_{p}\left(b^{\prime} x_{p}\right) x_{p}^{\prime}\right) \stackrel{(i i i)}{=}\left[x_{p} x_{p}^{\prime} \otimes x_{p}\right] \operatorname{vec}\left(b^{\prime}\right)=\left[x_{p} x_{p}^{\prime} \otimes x_{p}\right] b
$$




$$
\operatorname{vec}\left(x_{p}\left(x_{p}^{\prime} A x_{p}\right) x_{p}^{\prime}\right) \stackrel{(i i i)}{=}\left[x_{p} x_{p}^{\prime} \otimes x_{p} x_{p}^{\prime}\right] \operatorname{vec}(A) .
$$

Com essas últimas alterações, vetorizando a terceira equação, o sistema passa a ser

$$
\left\{\begin{array}{l}
\sum_{p \in \mathscr{P}} c+x_{p}^{\prime} b+\operatorname{vec}\left(x_{p} x_{p}^{\prime}\right)^{\prime} \operatorname{vec}(A)=\sum_{p \in \mathscr{P}} f\left(x_{p}\right) \\
\sum_{p \in \mathscr{P}} x_{p} c+\left(x_{p} x_{p}^{\prime}\right) b+x_{p} \operatorname{vec}\left(x_{p} x_{p}^{\prime}\right)^{\prime} \operatorname{vec}(A)=\sum_{p \in \mathscr{P}} f\left(x_{p}\right) x_{p} \\
\sum_{p \in \mathscr{P}} \operatorname{vec}\left(x_{p} x_{p}^{\prime}\right) c+\left[x_{p} x_{p}^{\prime} \otimes x_{p}\right] b+\left[x_{p} x_{p}^{\prime} \otimes x_{p} x_{p}^{\prime}\right] \operatorname{vec}(A)=\sum_{p \in \mathscr{P}} f\left(x_{p}\right) \operatorname{vec}\left(x_{p} x_{p}^{\prime}\right) .
\end{array}\right.
$$

Assim, finalmente, o sistema linear formado pode ser escrito como

$$
\sum_{p \in \mathscr{P}}\left[\begin{array}{ccc}
1 & x_{p}^{\prime} & \operatorname{vec}\left(x_{p} x_{p}^{\prime}\right)^{\prime} \\
x_{p} & x_{p} x_{p}^{\prime} & x_{p} \operatorname{vec}\left(x_{p} x_{p}^{\prime}\right)^{\prime} \\
\operatorname{vec}\left(x_{p} x_{p}^{\prime}\right) & {\left[x_{p} x_{p}^{\prime} \otimes x_{p}\right]} & {\left[x_{p} x_{p}^{\prime} \otimes x_{p} x_{p}^{\prime}\right]}
\end{array}\right]\left[\begin{array}{c}
c \\
b \\
\operatorname{vec}(A)
\end{array}\right]=\sum_{p \in \mathscr{P}}\left[\begin{array}{c}
f\left(x_{p}\right) \\
f\left(x_{p}\right) x_{p} \\
f\left(x_{p}\right) \operatorname{vec}\left(x_{p} x_{p}^{\prime}\right)
\end{array}\right] .
$$

Apenas uma observação final, como $A \in \mathbb{R}^{n \times n}, b \in \mathbb{R}^{n}$ e $c \in \mathbb{R}$, são necessários pelo menos $n^{2}+n+1$ pontos $x_{p}$ para que o problema fique bem definido (o menor valor de $P$ ). 

APÊNDICE

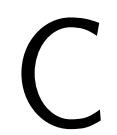

\section{MÉTODO DE DIREÇÕES CONJUGADAS}

Considere $f: \mathbb{R}^{n} \rightarrow \mathbb{R}$ uma função quadrática:

$$
\begin{gathered}
f(x)=\frac{1}{2} x^{\prime} A x+b^{\prime} x+c, \\
\nabla f(x)=A x+b .
\end{gathered}
$$

Sabe-se que, dependendo da caracterização de $A$, um ponto estacionário (minimizador ou maximizador) global $x^{*}$ é tal que

$$
A x^{*}=-b .
$$

Considerando que $A$ é positiva definida (portanto $A$ tem posto completo, é uma base que gera $\mathbb{R}^{n}$ e $x^{*}$ é o minimizador global da quadrática), pode-se conseguir uma base $\mathbf{D}=\left[d_{1}, \ldots, d_{n}\right] \not \supset$ 0 para $\mathbb{R}^{n}$ que seja $A$-conjugada/ortogonal, ou seja, $d_{i}^{\prime} A d_{j}=0$ para todo $i \neq j$. Obtida uma base D para $\mathbb{R}^{n}$ que seja $A$-ortogonal, qualquer ponto pode ser escrito como combinação linear dos elementos dessa base, e portanto,

$$
x^{*}=\alpha_{1} d_{1}+\alpha_{2} d_{2}+\cdots+\alpha_{n} d_{n}=\sum_{k=1}^{n} \alpha_{k} d_{k} .
$$

Pré-multiplicando a equação acima por $d_{i}^{\prime} A$, tem-se

$$
\begin{aligned}
d_{i}^{\prime} A x^{*} & =\alpha_{1} d_{i}^{\prime} A d_{1}+\alpha_{2} d_{i}^{\prime} A d_{2}+\cdots+\alpha_{n} d_{i}^{\prime} A d_{n} \\
& =\alpha_{i} d_{i}^{\prime} A d_{i},
\end{aligned}
$$

e então

$$
\alpha_{i}=\frac{d_{i}^{\prime} A x^{*}}{d_{i}^{\prime} A d_{i}} \stackrel{(\mathrm{C} .1)}{=}-\frac{d_{i}^{\prime} b}{d_{i}^{\prime} A d_{i}} .
$$

Assim, a solução ótima será

$$
x^{*} \stackrel{(\mathrm{C} .2),(\mathrm{C} .3)}{=} \sum_{k=1}^{n}-\left(\frac{d_{k}^{\prime} b}{d_{k}^{\prime} A d_{k}}\right) d_{k} .
$$




\section{C.1 Ortogonalização de Gram-Schmidt}

Dada uma matriz A simétrica positiva definida, objetivando obter uma base $\mathbf{B}$ que seja $A$-conjugada para o espaço $\mathbb{R}^{n}$ a partir de uma base $\mathbf{D}$ arbitrária (que pode ser a base canônica por exemplo), pode-se usar o processo de Gram-Schmidt.

Dada $\mathbf{D}=\left[d_{1}, \ldots, d_{n}\right]$ uma base de $\mathbb{R}^{n}$, ou seja, um conjunto 1.i., deseja-se obter uma base $\mathbf{B}=\left[b_{1}, \ldots, b_{n}\right]$ para $\mathbb{R}^{n}$ que seja $A$-conjugada, isto é, uma base em que $b_{i}^{\prime} A b_{j}=0$ para todo $i \neq j$. Assim, definem-se:

$$
\begin{aligned}
b_{1} & =d_{1} \\
b_{2} & =d_{2}-\frac{d_{2}^{\prime} A b_{1}}{b_{1}^{\prime} A b_{1}} b_{1} \\
b_{3} & =d_{3}-\frac{d_{3}^{\prime} A b_{1}}{b_{1}^{\prime} A b_{1}} b_{1}-\frac{d_{3}^{\prime} A b_{2}}{b_{2}^{\prime} A b_{2}} b_{2} \\
& \vdots \\
b_{n} & =d_{n}-\frac{d_{n}^{\prime} A b_{1}}{b_{1}^{\prime} A b_{1}} b_{1}-\frac{d_{n}^{\prime} A b_{2}}{b_{2}^{\prime} A b_{2}} b_{2}-\ldots-\frac{d_{n}^{\prime} A b_{n-1}}{b_{n-1}^{\prime} A b_{n-1}} b_{n-1} .
\end{aligned}
$$

Assim, B é uma base $A$-conjugada, e então pode-se aplicar o método de Direções Conjugadas, conforme descrito no Apêndice C.

Este processo pode ser feito por meio do Algoritmo 4.

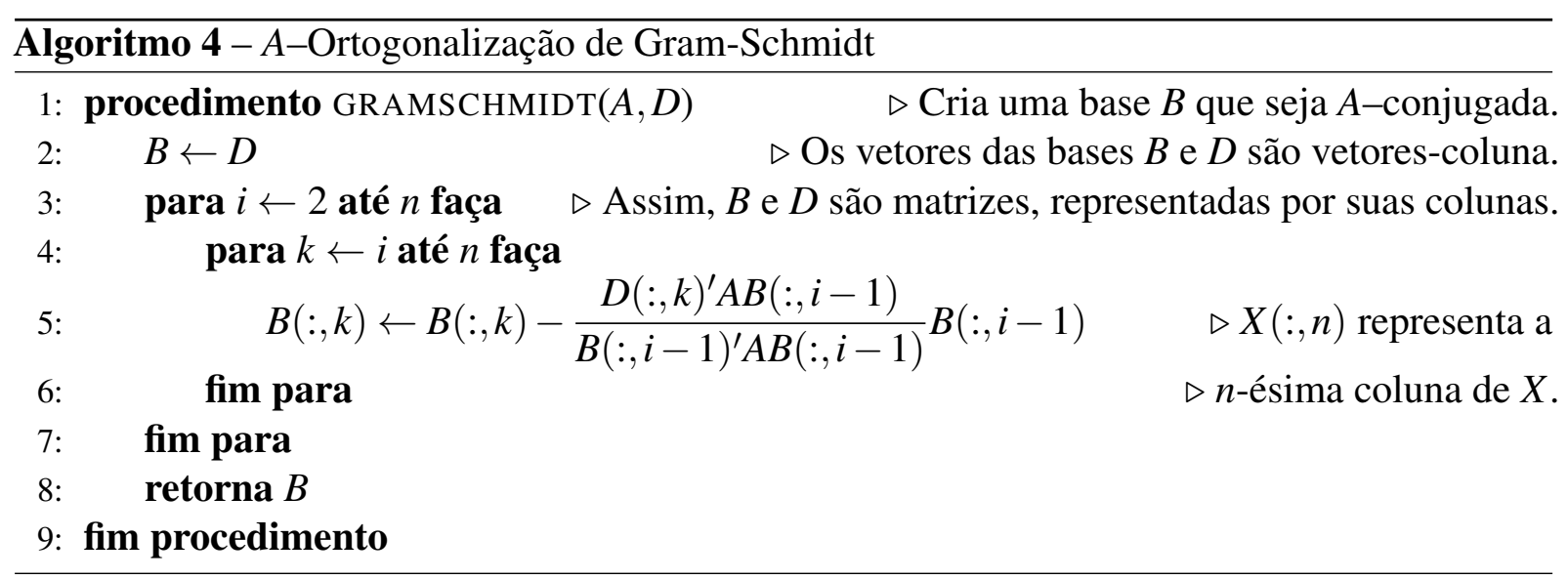

Linearmente independente. 
APÊNDICE

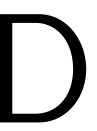

MAIS GRÁFICOS

Neste apêndice constam dados extras dos resultados, sob a forma de gráficos. Em todos os gráficos, o número de instâncias testadas é o mesmo, de 1000.

\section{D.1 Máscaras}

Figura 29 - Comparação dos custos com as máscaras e sem elas, com P1.

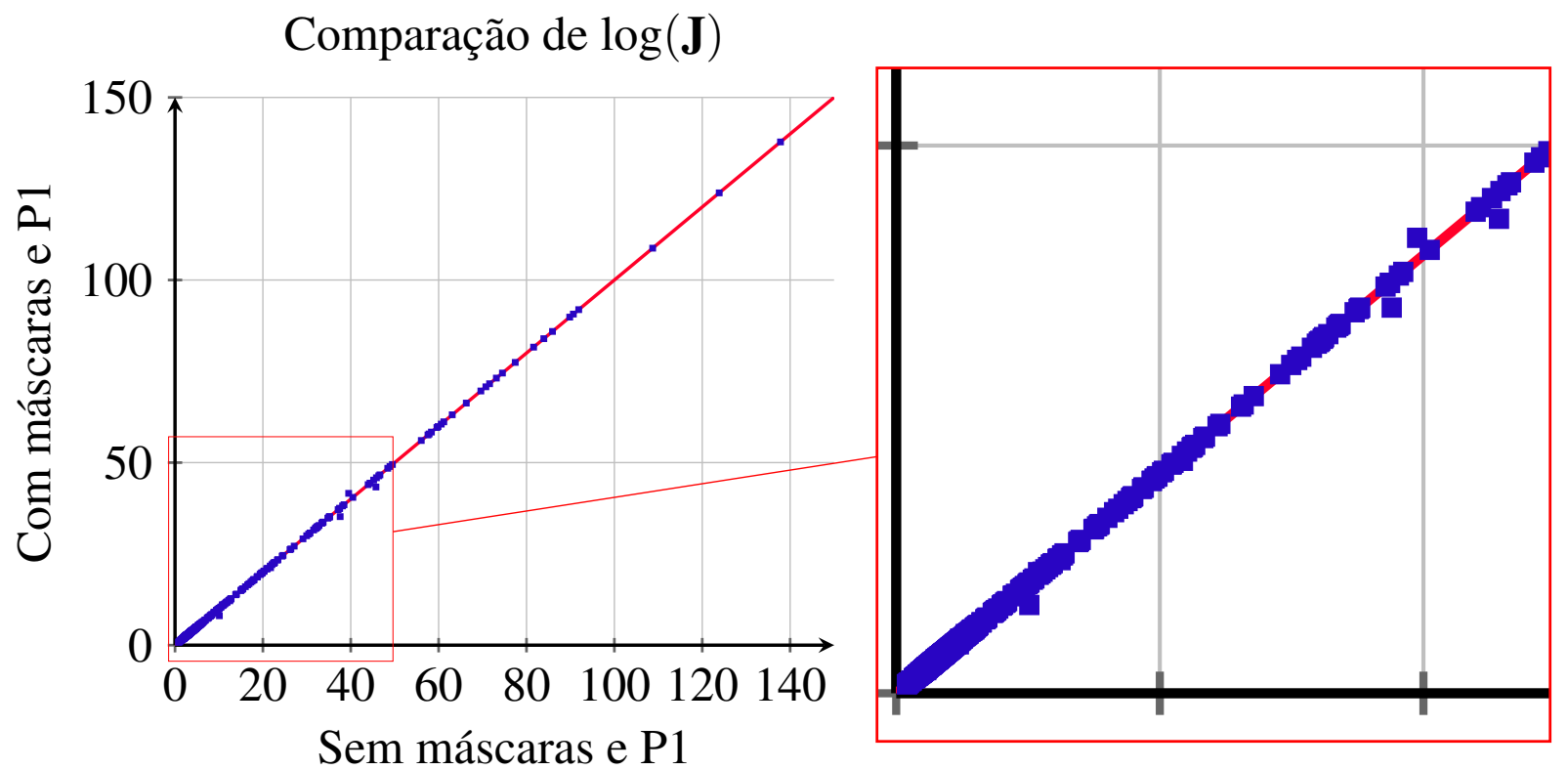


Figura 30 - Comparação dos custos com as máscaras e sem elas, com P2.

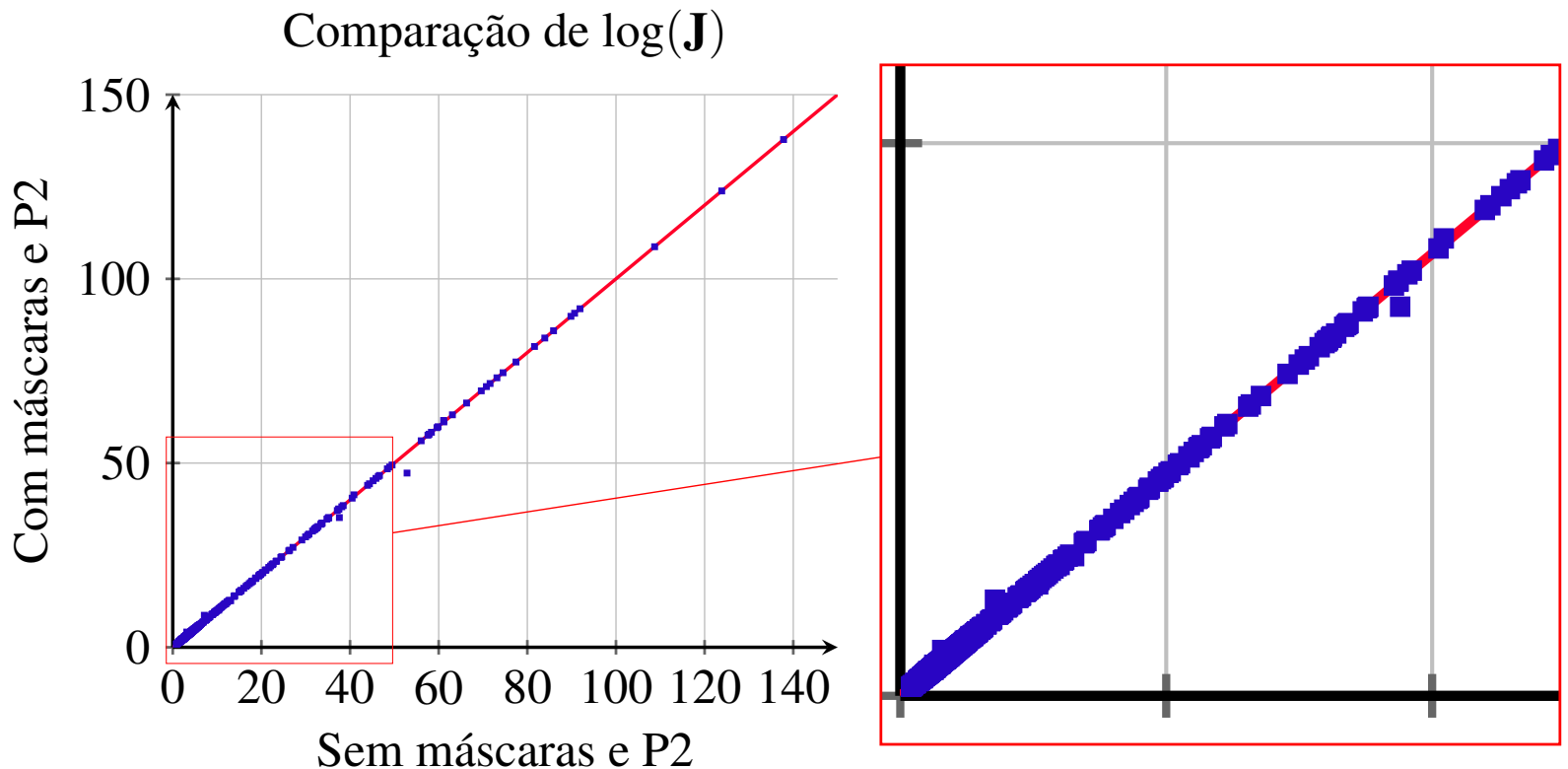

Figura 31 - Comparação dos custos com as máscaras e sem elas, com P3.

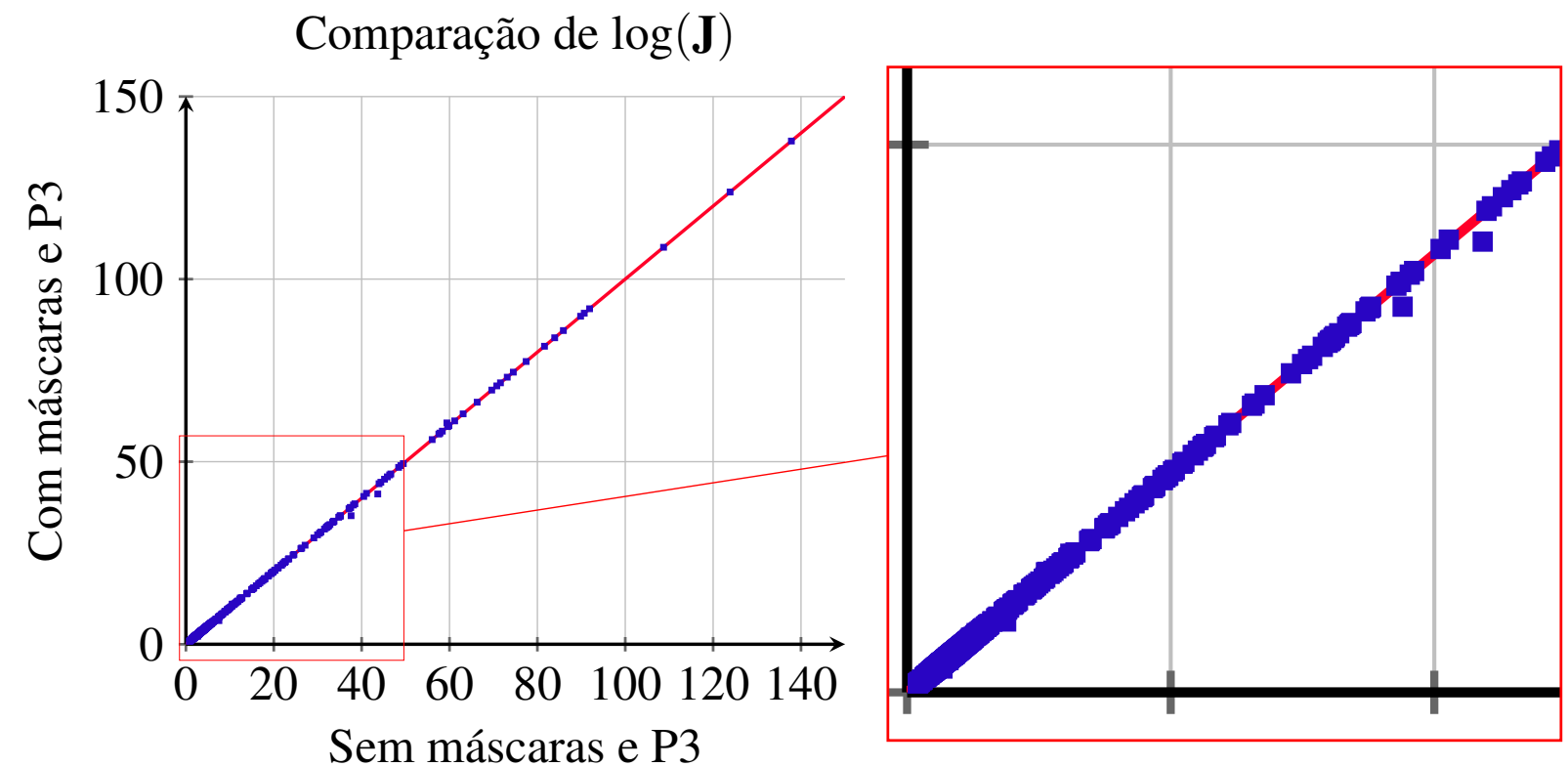


Figura 32 - Comparação dos custos com as máscaras e sem elas, com P4.
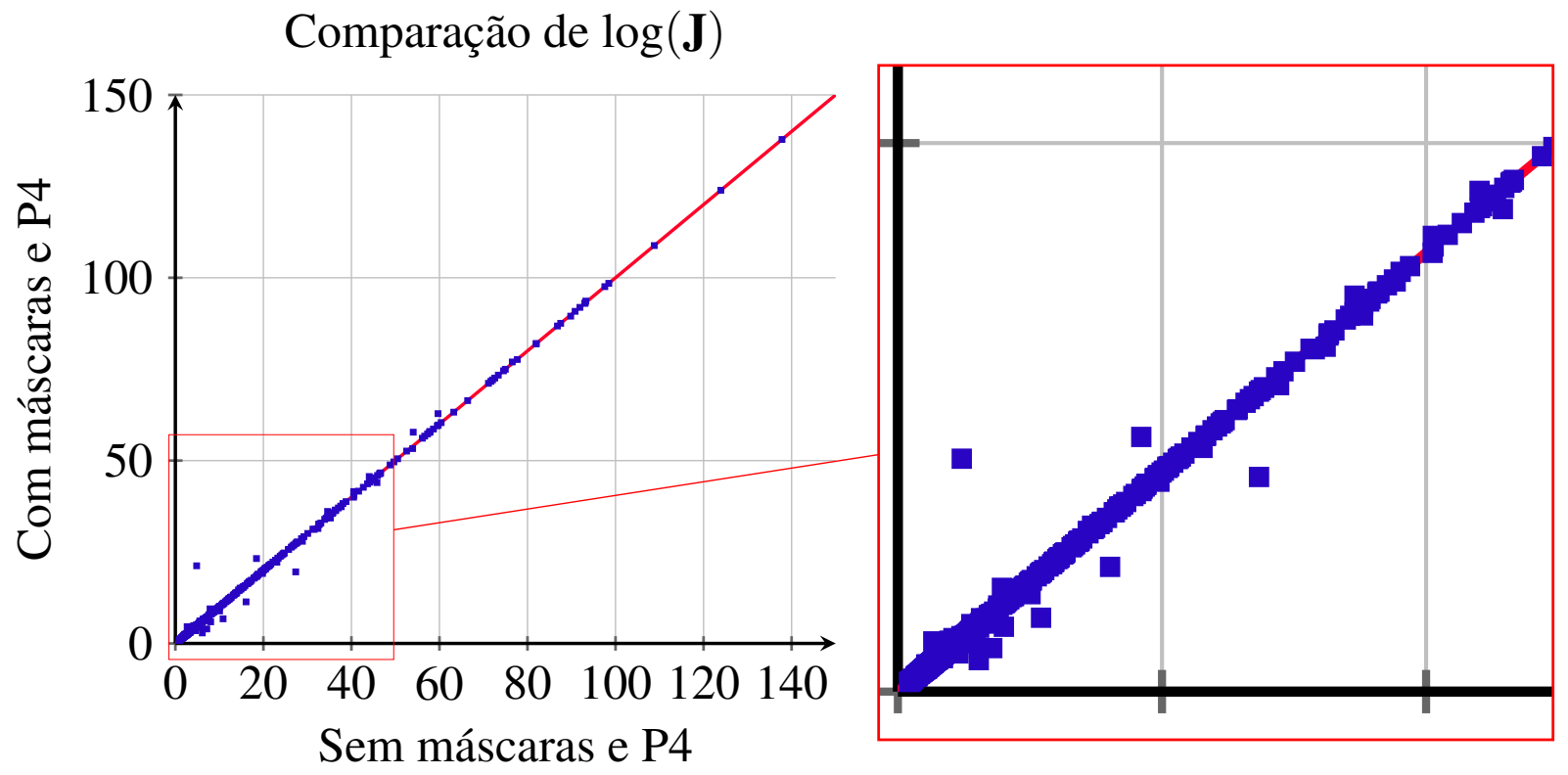

\section{D.2 Aproximação quadrática}

Figura 33 - Comparação dos custos com aproximação quadrática e sem ela, com P1.

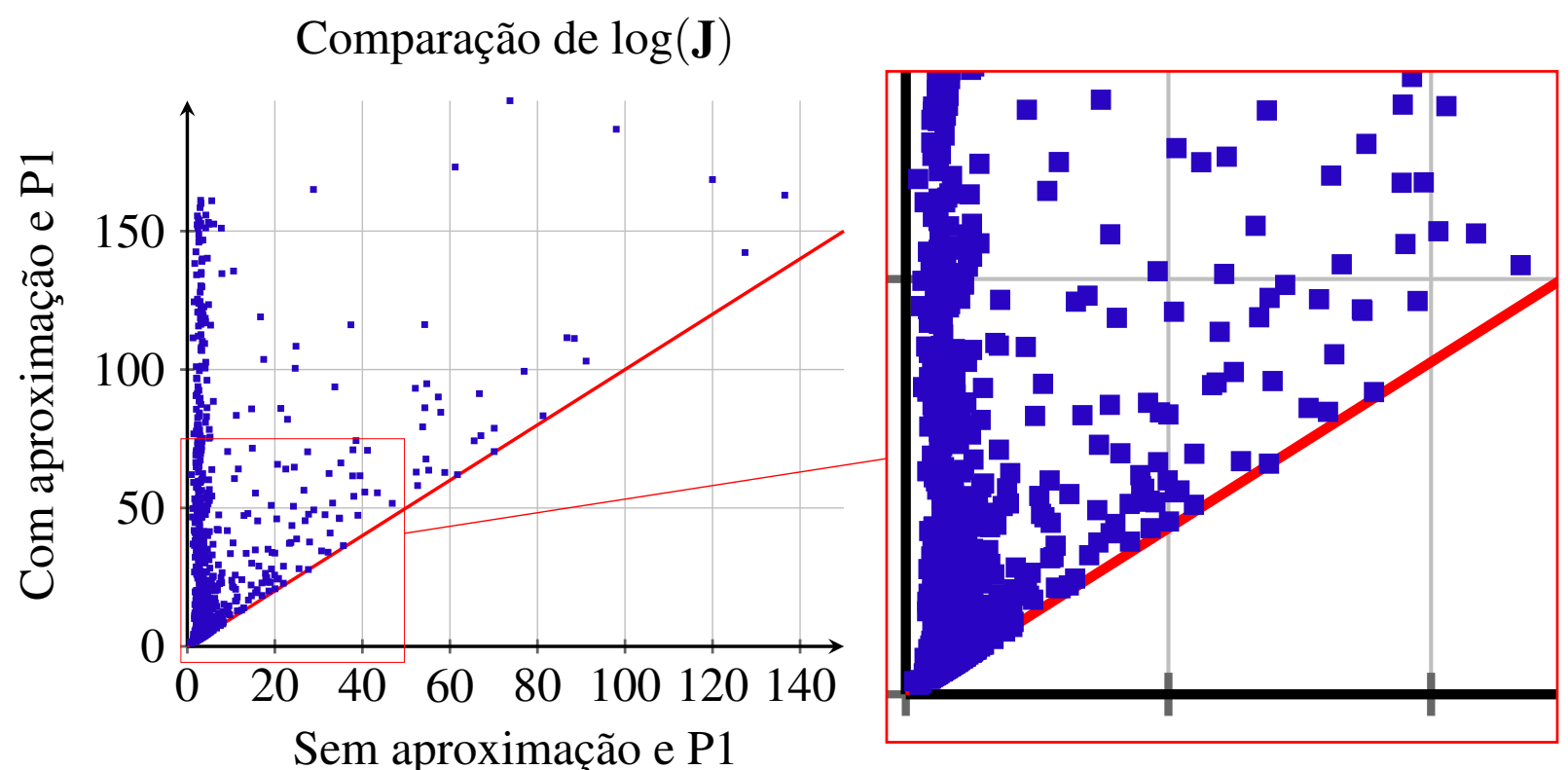


Figura 34 - Comparação dos custos com aproximação quadrática e sem ela, com P2.

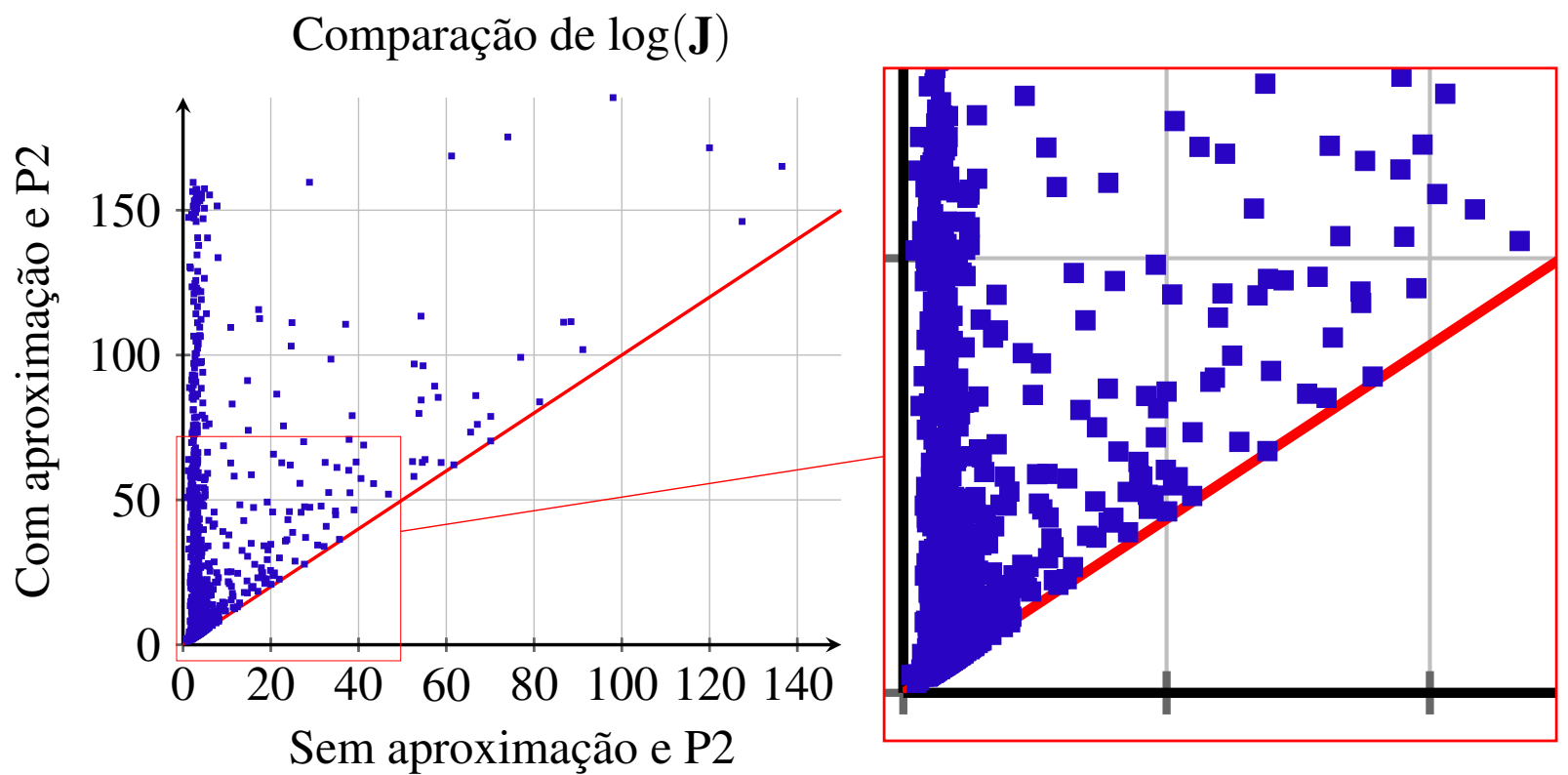

Figura 35 - Comparação dos custos com aproximação quadrática e sem ela, com P3.

Comparação de $\log (\mathbf{J})$
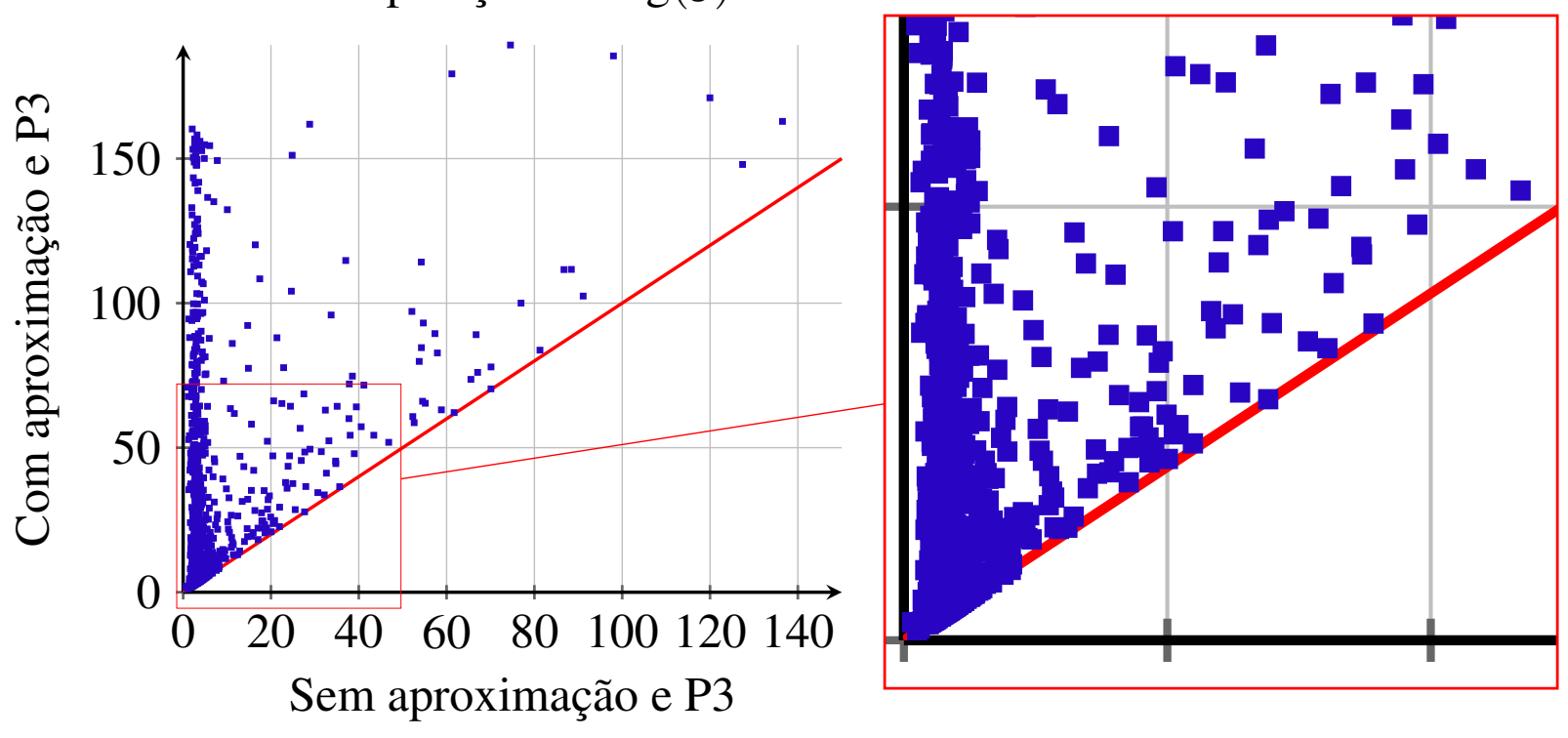
Figura 36 - Comparação dos custos com aproximação quadrática e sem ela, com P4.
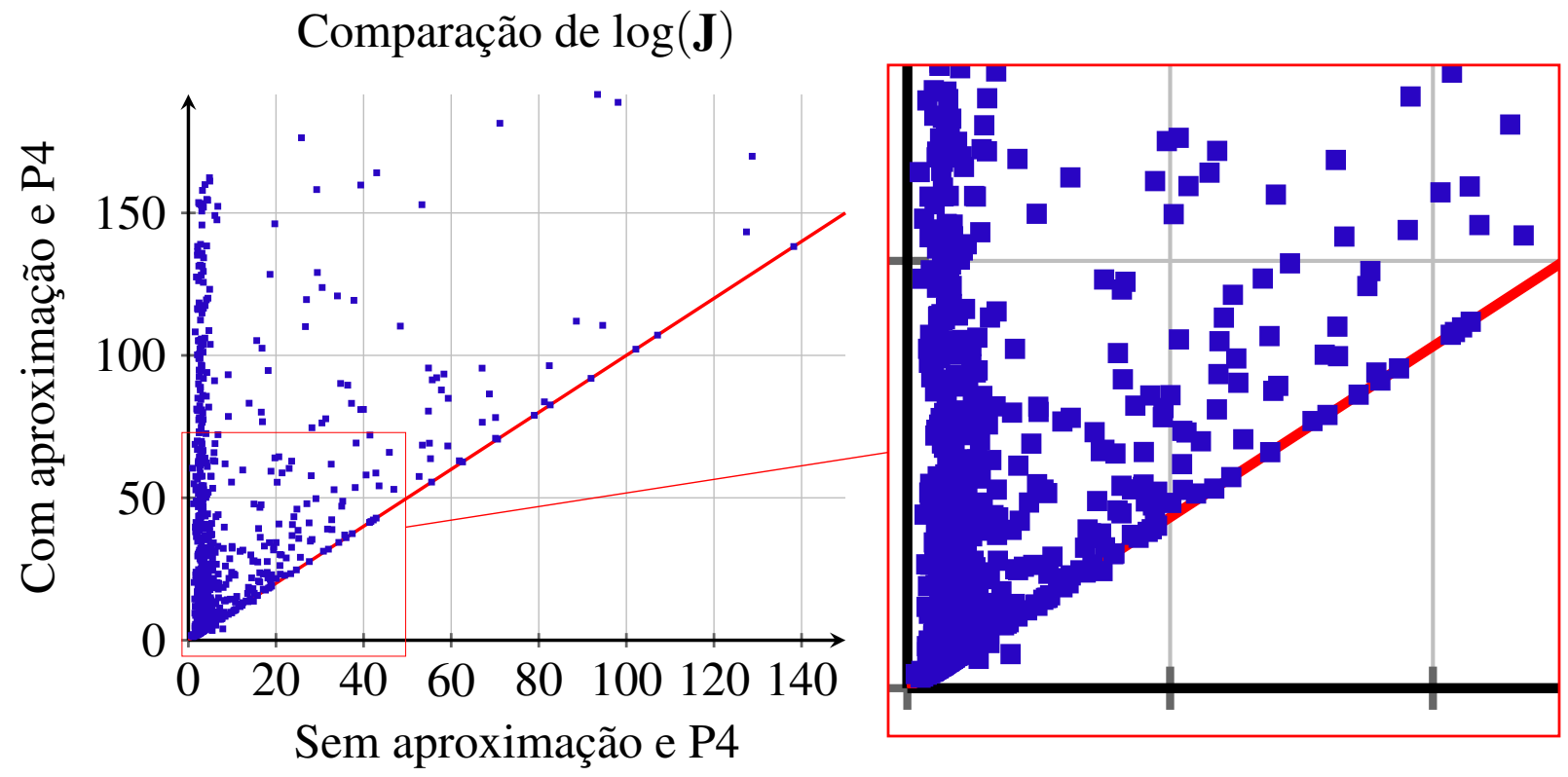

\section{D.3 Precondicionadores}

Figura 37 - Comparação dos custos com uso do P3 e sem ele.
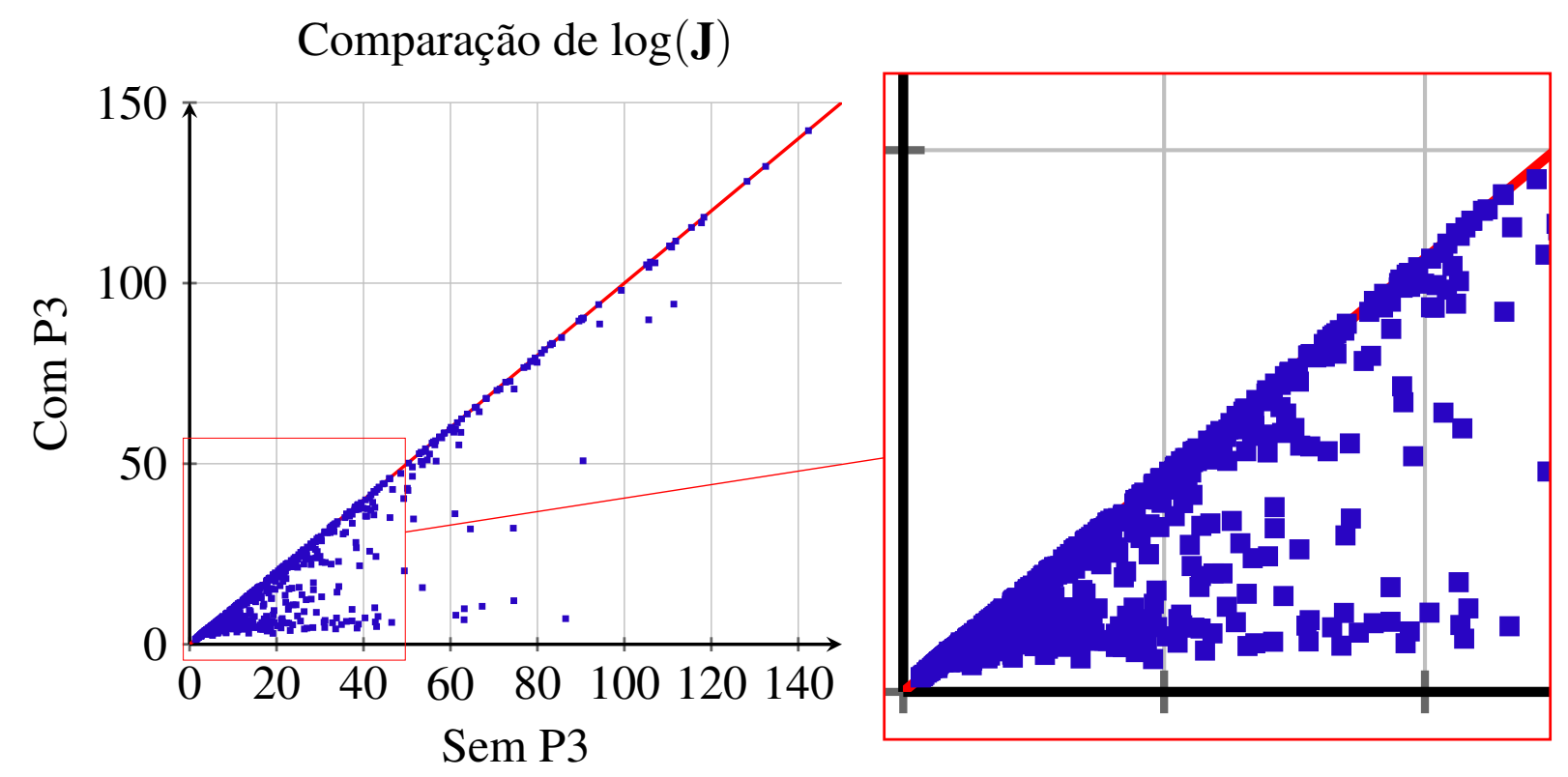
Figura 38 - Comparação dos custos com uso do P4 e sem ele.
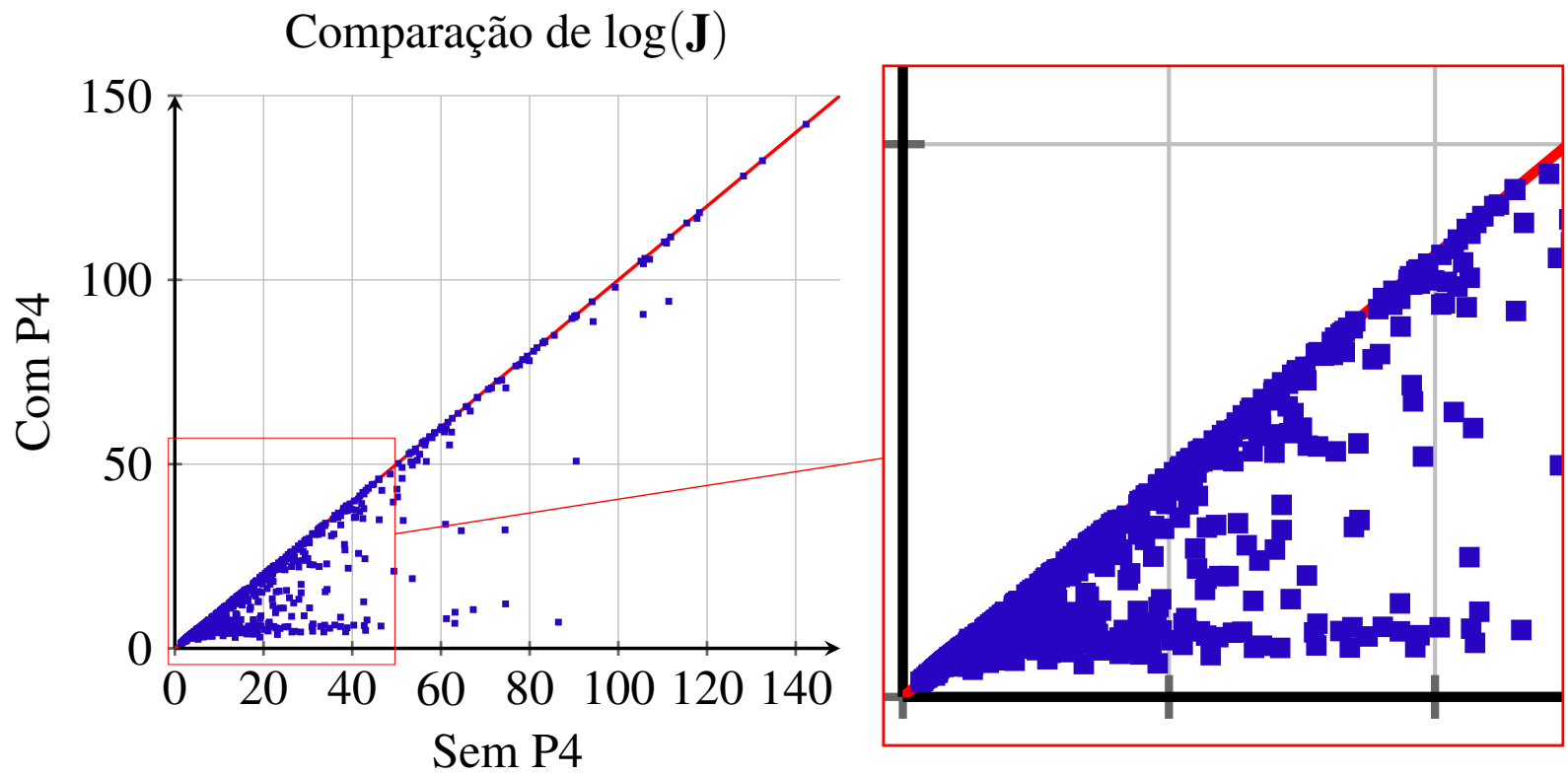

Figura 39 - Comparação dos custos com uso de P1 e P2.

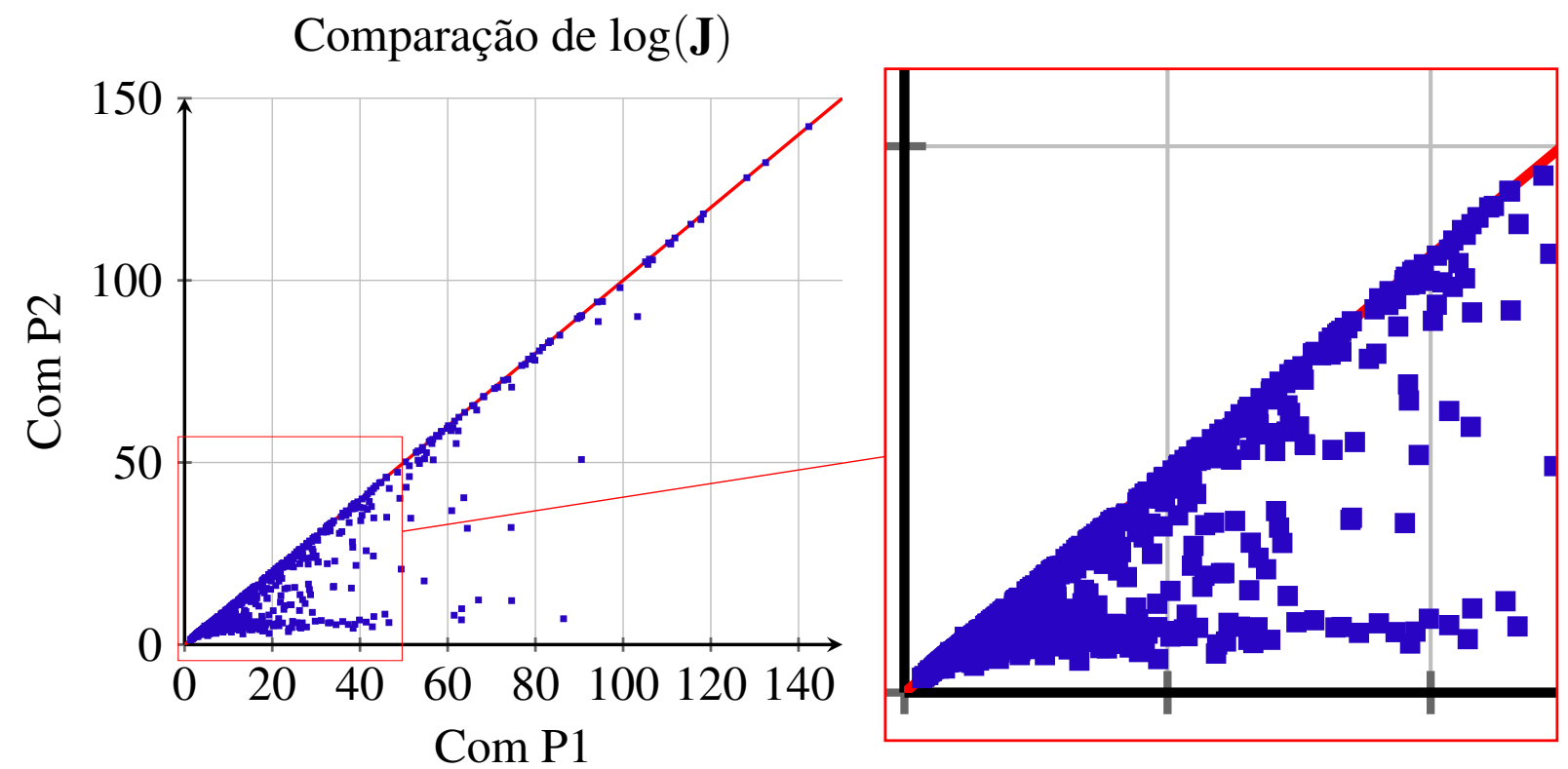


Figura 40 - Comparação dos custos com uso de P2 e P3.
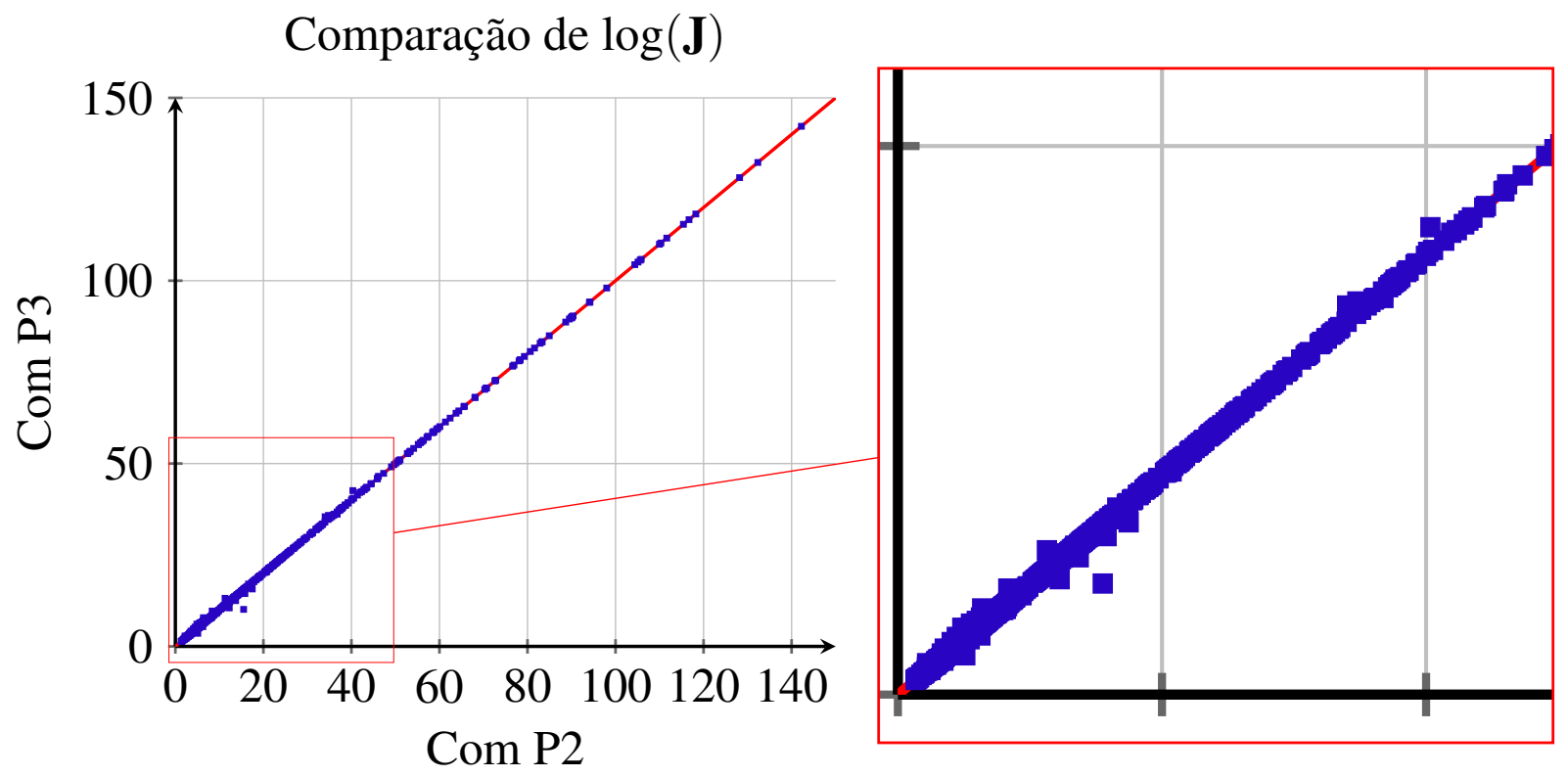

Figura 41 - Comparação dos custos com uso de P2 e P4.

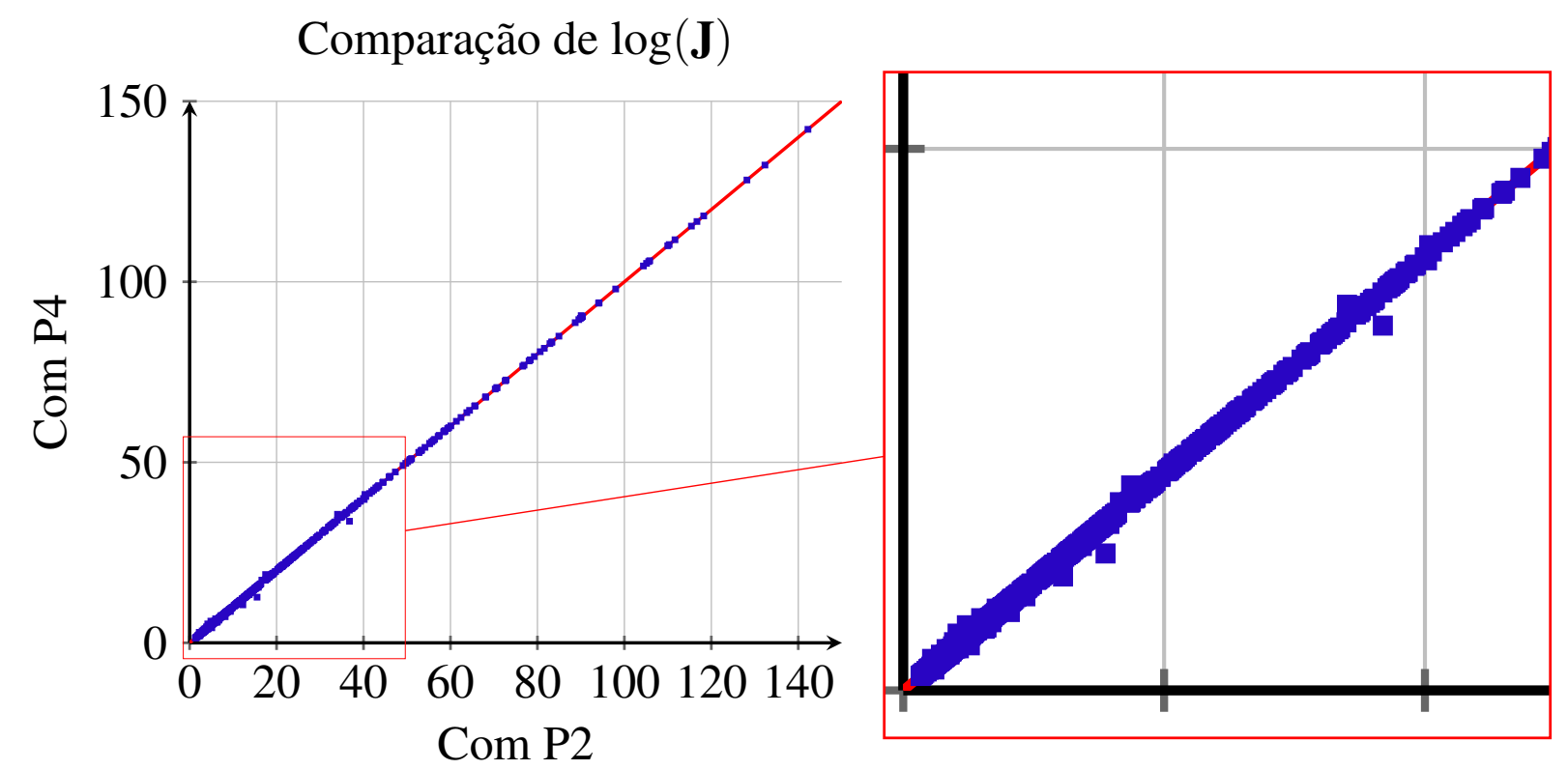


Figura 42 - Comparação dos custos com uso de P3 e P4.

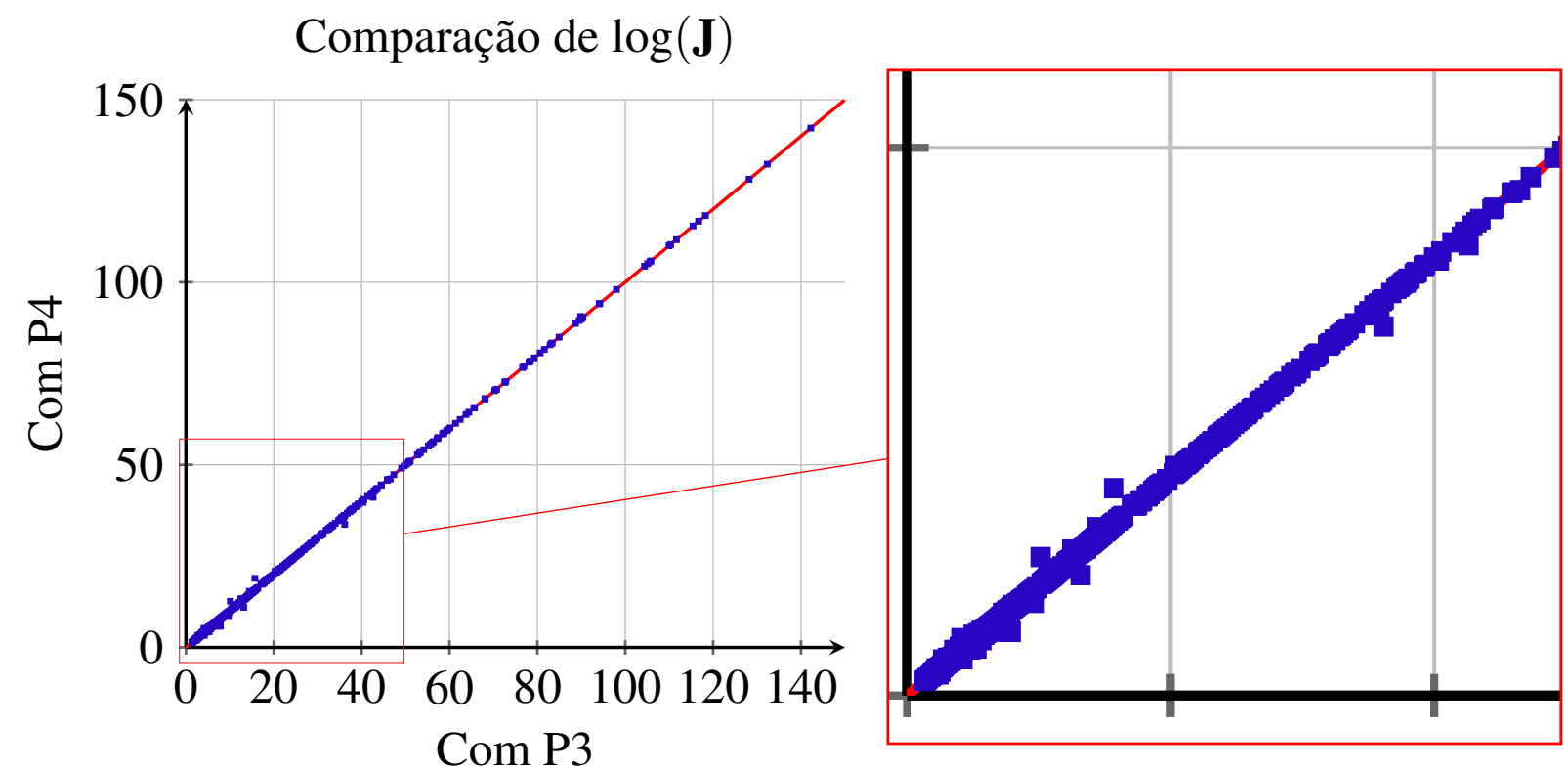




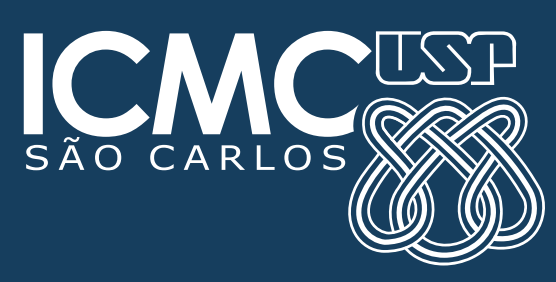

